\title{
Representation by Quaternary Quadratic Forms whose Coefficients are 1, 2, 7 or 14
}

by

Jamilah Alanazi, B. Math (King Faisal University)

A thesis submitted to the Faculty of Graduate and Postdoctoral Affairs in partial fulfillment of the requirements for the degree of Master of Science

School of Mathematics and Statistics Ottawa-Carleton Institute for Mathematics and Statistics Carleton University Ottawa, Ontario, Canada

(c) Copyright 2015, Jamilah Alanazi 


\section{Abstract}

We determine explicit formulae for the number of representations of a positive integer $n$ by the quaternary quadratic forms $a_{1} x_{1}^{2}+a_{2} x_{2}^{2}+a_{3} x_{3}^{2}+a_{4} x_{4}^{2}$, where $a_{1}, a_{2}, a_{3}, a_{4} \in$ $\{1,2,7,14\}$ which satisfy the simplifying assumptions $a_{1} \leq a_{2} \leq a_{3} \leq a_{4}$ and $\operatorname{gcd}\left(a_{1}, a_{2}, a_{3}, a_{4}\right)=1$. We use a modular form approach. We then extend our work to determine explicit formulae for the number of representations of $n$ by the octonary quadratic forms $x_{1}^{2}+x_{2}^{2}+x_{3}^{2}+x_{4}^{2}+7\left(x_{5}^{2}+x_{6}^{2}+x_{7}^{2}+x_{8}^{2}\right), x_{1}^{2}+x_{2}^{2}+7\left(x_{3}^{2}+x_{4}^{2}+x_{5}^{2}+x_{6}^{2}+x_{7}^{2}+x_{8}^{2}\right)$ and $x_{1}^{2}+x_{2}^{2}+x_{3}^{2}+x_{4}^{2}+x_{5}^{2}+x_{6}^{2}+7\left(x_{7}^{2}+x_{8}^{2}\right)$. 


\section{Dedication}

To my mother who was always supporting me and my husband Mohammed who never gave up. 


\section{Acknowledgements}

I thank God for everything that I have been blessed with in my lifespan. I would especially like to convey my special thanks to my supervisor Dr. Ayşe Alaca who has always been there for me, shown acceptance and a welcoming attitude towards me. I thank Dr. Şaban Alaca who have offered so much assistance during my program, as well as Carleton University and all the professors whose courses I have studied over the years. I also, thank Saudi Arabia for the financial support needed to do my Master's degree in Canada. 


\section{Contents}

Abstract $\quad$ i

Dedication $\quad$ ii

Acknowledgements $\quad$ iii

1 Introduction 1

2 Basic Concepts 4

2.1 Modular Forms . . . . . . . . . . . . . . . . . . . 4

2.2 Eisenstein Series . . . . . . . . . . . . . . . . . . . . . 9

2.3 Dimension Formulae . . . . . . . . . . . . . . . . . . . . . 10

3 Representations by Quaternary Quadratic Forms with Coefficients

$1,2,7$ and $14 \quad \mathbf{1 5}$

3.1 Preliminaries . . . . . . . . . . . . . . . . . . . 15

3.2 The space $M_{2}\left(\Gamma_{0}(56), \chi_{0}\right) \ldots \ldots \ldots \ldots \ldots$

3.3 The space $M_{2}\left(\Gamma_{0}(56), \chi_{3}\right) \ldots \ldots \ldots \ldots$

3.4 The space $M_{2}\left(\Gamma_{0}(56), \chi_{5}\right) \ldots \ldots \ldots \ldots$. . . . . . . . . . 41

3.5 The space $M_{2}\left(\Gamma_{0}(56), \chi_{6}\right) \ldots \ldots \ldots \ldots \ldots$ 
4 Representations by Octonary Quadratic Forms and Future Work 68

4.1 Representations by Octonary Quadratic Forms with Coefficients 1 and 768

4.2 Conclusion and Future Work . . . . . . . . . . . . . . . . . . 80 


\section{Chapter 1}

\section{Introduction}

Let $\mathbb{N}, \mathbb{N}_{0}, \mathbb{Z}, \mathbb{Q}, \mathbb{R}$ and $\mathbb{C}$ denote the sets of positive integers, nonnegative integers, integers, rational numbers, real numbers and complex numbers respectively. Let $a_{1}, a_{2}, a_{3}, a_{4} \in \mathbb{N}$, and $n \in \mathbb{N}_{0}$. Let $N\left(a_{1}, a_{2}, a_{3}, a_{4} ; n\right)$ denote the number of representations of $n$ by the quaternary quadratic form $a_{1} x_{1}^{2}+a_{2} x_{2}^{2}+a_{3} x_{3}^{2}+a_{4} x_{4}^{2}$, that is

$N\left(a_{1}, a_{2}, a_{3}, a_{4} ; n\right):=\operatorname{card}\left\{\left(x_{1}, x_{2}, x_{3}, x_{4}\right) \in \mathbb{Z}^{4} \mid n=a_{1} x_{1}^{2}+a_{2} x_{2}^{2}+a_{3} x_{3}^{2}+a_{4} x_{4}^{2}\right\}$.

It is clear that $N\left(a_{1}, a_{2}, a_{3}, a_{4} ; 0\right)=1$. Since $N\left(a_{1}, a_{2}, a_{3}, a_{4} ; n\right)$ is invariant under a permutation of $a_{1}, a_{2}, a_{3}, a_{4}$, we may suppose that

$$
a_{1} \leq a_{2} \leq a_{3} \leq a_{4}
$$

Note that if $\operatorname{gcd}\left(a_{1}, a_{2}, a_{3}, a_{4}\right)=d$, then $N\left(a_{1}, a_{2}, a_{3}, a_{4} ; n\right)=N\left(a_{1} / d, a_{2} / d, a_{3} / d, a_{4} / d ; n / d\right)$. So we may also suppose that

$$
\operatorname{gcd}\left(a_{1}, a_{2}, a_{3}, a_{4}\right)=1
$$


Our first objective in this thesis is to determine explicit formulae for $N\left(a_{1}, a_{2}, a_{3}, a_{4} ; n\right)$, where $a_{1}, a_{2}, a_{3}, a_{4} \in\{1,2,7,14\}$ which satisfy the simplifying assumptions (1.0.1) and (1.0.2).

We then extend our work to determine explicit formulae for the number of representations of $n$ by the octonary quadratic forms $x_{1}^{2}+x_{2}^{2}+x_{3}^{2}+x_{4}^{2}+7\left(x_{5}^{2}+x_{6}^{2}+x_{7}^{2}+x_{8}^{2}\right)$, $x_{1}^{2}+x_{2}^{2}+7\left(x_{3}^{2}+x_{4}^{2}+x_{5}^{2}+x_{6}^{2}+x_{7}^{2}+x_{8}^{2}\right)$ and $x_{1}^{2}+x_{2}^{2}+x_{3}^{2}+x_{4}^{2}+x_{5}^{2}+x_{6}^{2}+7\left(x_{7}^{2}+x_{8}^{2}\right)$, which we denote by $N\left(1^{4}, 7^{4} ; n\right), N\left(1^{2}, 7^{6} ; n\right)$ and $N\left(1^{6}, 7^{2} ; n\right)$ respectively.

Over the years many people have worked on the problem of representations of integers by quadratic forms. In 1770 Lagrange [16] proved that every positive integer can be written as a sum of four integer squares.

Jacobi [12] gave formulae for $N(1,1,1,1 ; n)$ as

$$
N(1,1,1,1 ; n)=8 \sigma(n)-32 \sigma(n / 4)= \begin{cases}8 \sigma(n) & \text { if } 4 \nmid n, \\ 24 \sigma(n) & \text { if } 4 \mid n,\end{cases}
$$

where $\sigma(n)$ is the sum of divisors function. See [26]. In 1860, Liouville [18] gave a formula for $N(1,1,2,2 ; n)$ and in 1861 he gave two more formulae for $N(1,1,1,2 ; n)$ and $N(1,2,2,2 ; n)$. Also, Benz [4], Demuth [10], and Pepin [23] gave proofs for these formulae. Williams [29] gave a completely arithmetic proof of the Liouville formulae for $N(1,1,1,2 ; n)$ and $N(1,2,2,2 ; n)$.

In Chapter 2 we present some basic properties of modular groups and modular forms. In Chapter 3 we determine an explicit formula for $N\left(a_{1}, a_{2}, a_{3}, a_{4} ; n\right)$ for each of the twenty-two quaternary quadratic forms given by

$$
\begin{aligned}
\left(a_{1}, a_{2}, a_{3}, a_{4}\right)= & (1,1,7,7),(2,2,7,7),(1,2,7,14),(1,1,14,14),(1,1,1,7), \\
& (1,2,2,7),(1,7,7,7),(1,1,2,14),(2,7,7,14),(1,7,14,14),
\end{aligned}
$$




$$
\begin{aligned}
& (1,1,2,7),(2,2,2,7),(2,7,7,7),(1,1,1,14),(1,2,2,14), \\
& (1,7,7,14),(2,7,14,14),(1,14,14,14),(1,2,7,7),(1,1,7,14), \\
& (2,2,7,14),(1,2,14,14) .
\end{aligned}
$$

To the best of our knowledge, these are the only remaining diagonal quaternary quadratic forms with coefficients 1, 2, 7 and 14 for which explicit formulae for $N\left(a_{1}, a_{2}, a_{3}, a_{4} ; n\right)$ have not been determined so far. In Chapter 4 we determine the number of representations of a positive integer $n$ by the octonary quadratic forms $N\left(1^{4}, 7^{4} ; n\right), N\left(1^{2}, 7^{6} ; n\right)$ and $N\left(1^{6}, 7^{2} ; n\right)$. We conclude our thesis by indicating some further directions for our research. 


\section{Chapter 2}

\section{Basic Concepts}

In this chapter we present some basic concepts for modular forms. For more information one can see [8], [9], [14], [15], [21], [22], [25], [27], and [28].

\subsection{Modular Forms}

Definition 2.1.1. The modular group $S L_{2}(\mathbb{Z})$ is defined as

$$
S L_{2}(\mathbb{Z})=\left\{\left(\begin{array}{ll}
a & b \\
c & d
\end{array}\right) \mid a, b, c, d \in \mathbb{Z}, a d-b c=1\right\}
$$

which acts on the upper half plane $\mathbb{H}=\{z \in \mathbb{C} \mid \operatorname{Im}(z)>0\}$ by the linear fractional transformation

$$
\left(\begin{array}{ll}
a & b \\
c & d
\end{array}\right)(z)=\frac{a z+b}{c z+d}, \text { for } z \in \mathbb{H}
$$


Note that the modular group $S L_{2}(\mathbb{Z})$ is generated by two elements $T=\left(\begin{array}{cc}1 & 1 \\ 0 & 1\end{array}\right)$ and $S=\left(\begin{array}{cc}0 & -1 \\ 1 & 0\end{array}\right)$.

Definition 2.1.2. Let $N \in \mathbb{N}$. We define the principal congruence subgroup $\Gamma(N)$ by

$$
\Gamma(N):=\left\{\left(\begin{array}{ll}
a & b \\
c & d
\end{array}\right) \in S L_{2}(\mathbb{Z}) \mid a \equiv d \equiv 1(\bmod \mathrm{N}), b \equiv c \equiv 0(\bmod \mathrm{N})\right\}
$$

A subgroup $\Gamma$ of $S L_{2}(\mathbb{Z})$ is called a congruence subgroup if it contains $\Gamma(N)$ for some positive integer $N$. The smallest such $N$ is called the level of $\Gamma$. The two important congruence subgroups are

$$
\begin{gathered}
\Gamma_{0}(N):=\left\{\left(\begin{array}{ll}
a & b \\
c & d
\end{array}\right) \in S L_{2}(\mathbb{Z}) \mid c \equiv 0(\bmod \mathrm{N})\right\}, \\
\Gamma_{1}(N):=\left\{\left(\begin{array}{ll}
a & b \\
c & d
\end{array}\right) \in \Gamma_{0}(N) \mid a \equiv d \equiv 1(\bmod \mathrm{N})\right\} .
\end{gathered}
$$

Definition 2.1.3. A Dirichlet character $(\bmod N)$ is a function $\chi: \mathbb{Z} \rightarrow C$ satisfying

(i) $\chi(a b)=\chi(a) \chi(b)$ for any $a, b \in \mathbb{Z}$,

(ii) $\chi(a) \neq 0$ if $\operatorname{gcd}(a, N)=1$,

(iii) $\chi(a)=0$ if $\operatorname{gcd}(a, N)>1$

(iv) $\chi(a)=\chi(b)$ if $a \equiv b(\bmod N)$.

Definition 2.1.4. The trivial character is the Dirichlet character of modulus 1 and is denoted by $\chi_{0}$.

Definition 2.1.5. The conductor of a Dirichlet character $\chi$ is the smallest positive integer $M$ dividing its modulus such that there exists a Dirichlet character $\psi$ of 
modulus $M$ with $\chi(a)=\psi(a)$ for all $a \in \mathbb{Z}$ with $(a, N)=1$. We say that a Dirichlet character modulo $N$ is primitive if its conductor equals its modulus.

Definition 2.1.6. Let $k \in \mathbb{Z}$. A weakly modular function of weight $k$ for a congruence subgroup $\Gamma$ is a meromorphic function $f: \mathbb{H} \rightarrow \mathbb{C}$ which satisfies

$$
f\left(\frac{a z+b}{c z+d}\right)=(c z+d)^{k} f(z), \text { for }\left(\begin{array}{ll}
a & b \\
c & d
\end{array}\right) \in \Gamma \text { and } z \in \mathbb{H} .
$$

Definition 2.1.7. [14] Let $\gamma=\left(\begin{array}{ll}a & b \\ c & d\end{array}\right) \in \Gamma$ and $z \in \mathbb{H}$. We define the weight $k$ operator $\mid[\gamma]_{k}$ on a function from $\mathbb{H}$ to $\mathbb{C}$ by

$$
\left(f \mid[\gamma]_{k}\right)(z)=(c z+d)^{-k} f\left(\frac{a z+b}{c z+d}\right) .
$$

Definition 2.1.8. [14] Let $\Gamma$ be a congruence subgroup of level $N$ in $S L_{2}(\mathbb{Z})$ and $k \in \mathbb{Z}$. A function $f: \mathbb{H} \rightarrow \mathbb{C}$ is called a modular form of weight $k$ for $\Gamma$ if it satisfies the following conditions

(i) $f$ is weakly modular for $\Gamma$,

(ii) $f$ is holomorphic on $\mathbb{H}$,

(iii) $f \mid[\alpha]_{k}$ is holomorphic at $\infty$ for all $\alpha \in S L_{2}(\mathbb{Z})$.

Note that by condition (iii), $f \mid[\alpha]_{k}$ has a Fourier expansion of the form

$$
f \mid[\alpha]_{k}=\sum_{n=0}^{\infty} a_{n} q_{N}^{n}, \quad q_{N}=e^{2 \pi i z / N} .
$$

We say that $f$ is a cusp form of weight $k$ for $\Gamma$ if $f \mid[\alpha]_{k}$ vanishes at $\infty$, which mean $a_{0}=0$ for every $\alpha \in S L_{2}(\mathbb{Z})$ in the Fourier expansion of $f \mid[\alpha]_{k}$.

Definition 2.1.9. [14] Let $N$ be a positive integer and let $\chi$ be a Dirichlet character. 
A function $f: \mathbb{H} \rightarrow \mathbb{C}$ which is holomorphic on $\mathbb{H}$ and $f \mid[\alpha]_{k}$ is holomorphic at $\infty$ for all $\alpha$ is a modular form of weight $k$ for $\Gamma_{0}(N)$ with character $\chi$ if

$$
f \mid[\gamma]_{k}=\chi(d) f(z) \text { for all } \gamma=\left(\begin{array}{ll}
a & b \\
c & d
\end{array}\right) \in \Gamma_{0}(N) .
$$

We write $M_{k}\left(\Gamma_{0}(N), \chi\right)$ to denote the space of modular forms of weight $k$ and character $\chi$, and $S_{k}\left(\Gamma_{0}(N), \chi\right)$ to denote the subspace of cusp forms of weight $k$ and character $\chi$.

Let $k \in \mathbb{Z}$. We write $E_{k}\left(\Gamma_{0}(N), \chi\right)$ to denote the subspace of Eisenstein series. It is known (see for example $[28, \mathrm{p} .83]$ ) that

$$
M_{k}\left(\Gamma_{0}(N), \chi\right)=E_{k}\left(\Gamma_{0}(N), \chi\right) \oplus S_{k}\left(\Gamma_{0}(N), \chi\right) .
$$

Definition 2.1.10. The Dedekind eta function is defined on the upper half plane $\mathbb{H}$ by the product formula

$$
\eta(z)=q^{1 / 24} \prod_{n=1}^{\infty}\left(1-q^{n}\right), q=e^{2 \pi i z} .
$$

An eta quotient is defined as a finite product of the form

$$
f(z)=\prod_{\delta} \eta^{r_{\delta}}(\delta z)
$$

where $\delta$ runs through a finite set of positive integers and the exponents $r_{\delta}$ are non-zero integers. By taking $N$ to be the least common multiple of the $\delta^{\prime} s$ we can write the 
eta quotient (2.1.3) as

$$
f(z)=\prod_{1 \leq \delta \mid N} \eta^{r_{\delta}}(\delta z)
$$

where some of the exponents $r_{\delta}$ may be 0 . When all exponents are non-negative, $f(z)$ is said to be an eta product. For $q \in \mathbb{C}$ with $|q|<1$ we set

$$
F(q)=\prod_{n=1}^{\infty}\left(1-q^{n}\right)
$$

Appealing to (2.1.5) we can express the eta function (2.1.2) as

$$
\eta(z)=q^{1 / 24} \prod_{n=1}^{\infty}\left(1-q^{n}\right)=q^{1 / 24} F(q), q=e^{2 \pi i z} .
$$

Definition 2.1.11. For $q \in \mathbb{C}$ with $|q|<1$ Ramanujan's theta function $\varphi(q)$ is defined by

$$
\varphi(q)=\sum_{n=-\infty}^{\infty} q^{n^{2}} .
$$

We note that for quaternary quadratic forms $a_{1} x_{1}^{2}+a_{2} x_{2}^{2}+a_{3} x_{3}^{2}+a_{4} x_{4}^{2}\left(a_{1}, a_{2}, a_{3}, a_{4} \in\right.$ $\mathbb{N}$ ), we have

$$
\sum_{n=0}^{\infty} N\left(a_{1}, a_{2}, a_{3}, a_{4} ; n\right) q^{n}=\varphi\left(q^{a_{1}}\right) \varphi\left(q^{a_{2}}\right) \varphi\left(q^{a_{3}}\right) \varphi\left(q^{a_{4}}\right)
$$

and for octonary quadratic forms $a_{1} x_{1}^{2}+\cdots+a_{8} x_{8}^{2}\left(a_{1}, \ldots, a_{8} \in \mathbb{N}\right)$, we have

$$
\sum_{n=0}^{\infty} N\left(a_{1}, \ldots, a_{8} ; n\right) q^{n}=\varphi\left(q^{a_{1}}\right) \cdots \varphi\left(q^{a_{8}}\right) .
$$


The infinite product representation of $\varphi(q)$ is due to Jacobi $([3])$,

$$
\varphi(q)=\frac{F^{5}\left(q^{2}\right)}{F^{2}(q) F^{2}\left(q^{4}\right)},
$$

where $F(q)$ is given by (2.1.5). It follows from (2.1.2), (2.1.5) and (2.1.8) that

$$
\eta(z)=\frac{\eta^{5}(2 z)}{\eta^{2}(z) \eta^{2}(4 z)} .
$$

\subsection{Eisenstein Series}

Definition 2.2.1. Let $\chi$ and $\psi$ be Dirichlet characters. For $n \in \mathbb{N}$ we define $\sigma_{(k-1, \chi, \psi)}(n)$ by

$$
\sigma_{(k-1, \chi, \psi)}(n):=\sum_{1 \leq m \mid n} \psi(m) \chi(n / m) m^{k-1}
$$

We set $\sigma_{(k-1, \chi, \psi)}(n)=0$ for $n \notin \mathbb{N}$. If $\chi$ and $\psi$ are trivial characters then $\sigma_{(k-1, \chi, \psi)}(n)$ becomes the sum of divisors function

$$
\sigma_{k-1}(n)=\sum_{1 \leq m \mid n} m^{k-1} .
$$

Definition 2.2.2. Let $\psi$ be a Dirichlet character of modulus $N$. We define the generalized Bernoulli numbers $\left\{B_{k, \psi}\right\}_{k \in \mathbb{N}}$ by the formal series

$$
\sum_{a=1}^{N} \frac{x e^{a x} \psi(a)}{e^{N x}-1}=\sum_{k=0}^{\infty} B_{k, \psi} \frac{x^{k}}{k !}
$$

Let $\chi$ and $\psi$ be primitive Dirichlet characters with conductors $L$ and $M$, respec- 
tively. We set

$$
E_{k, \chi, \psi}(q)=c_{0}+\sum_{n \geq 1}\left(\sum_{m \mid n} \psi(m) \chi(n / m) m^{k-1}\right) q^{n},
$$

where $c_{0}$ is written in terms of the generalized Bernoulli numbers defined by

$$
c_{0}= \begin{cases}0 & \text { if } L>1 \\ -\frac{B_{k, \psi}}{2 k} & \text { if } L=1\end{cases}
$$

If $\chi$ and $\psi$ are trivial characters, then the Eisenstein series $E_{2, \chi_{0}, \chi_{0}}(q)$ and $E_{4, \chi_{0}, \chi_{0}}(q)$ become

$$
L(q):=E_{2}(q):=E_{2, \chi_{0}, \chi_{0}}(q)=-\frac{1}{24}+\sum_{n=1}^{\infty} \sigma(n) q^{n}
$$

and

$$
E_{4}(q):=E_{4, \chi_{0}, \chi_{0}}(q)=\frac{1}{240}+\sum_{n=1}^{\infty} \sigma_{3}(n) q^{n} .
$$

The following theorem can be found in [28].

Theorem 2.2.3. Suppose $t, k$ are positive integers. Let $\chi$ and $\psi$ be Dirichlet characters with conductors $L$ and $M$, respectively. The power series $E_{k, \chi, \psi}\left(q^{t}\right)$ with $L M t \mid N$ and $\chi \psi=\varepsilon$ form a basis for the Eisenstein subspace $E_{k}\left(\Gamma_{0}(N), \varepsilon\right)$. Except if $k=2$, $\chi=\psi=1, t>1$ then $L(q)-t L\left(q^{t}\right)$ is a modular form of weight 2 in $M_{2}\left(\Gamma_{0}(t)\right)$.

\subsection{Dimension Formulae}

Let $N, k \in \mathbb{N}$ and $\chi$ a Dirichlet character. In this section we state formulae for the dimensions of $M_{k}\left(\Gamma_{0}(N), \chi\right), E_{k}\left(\Gamma_{0}(N), \chi\right)$ and $S_{k}\left(\Gamma_{0}(N), \chi\right)$. First, we state the 
trivial character case. Let

$$
\begin{aligned}
& \mu_{0}(N)=N \prod_{p \mid N}(1+1 / p), \\
& \mu_{0,2}(N)= \begin{cases}0 & \text { if } 4 \mid N, \\
\prod_{p \mid N}\left(1+\left(\frac{-4}{p}\right)\right) & \text { otherwise, }\end{cases} \\
& \mu_{0,3}(N)= \begin{cases}0 & \text { if } 2 \mid N \text { or } 9 \mid N, \\
\prod_{p \mid N}\left(1+\left(\frac{-3}{p}\right)\right) & \text { otherwise, }\end{cases} \\
& c_{0}(N)=\sum_{d \mid N} \phi(\operatorname{gcd}(d, N / d)),
\end{aligned}
$$

where $\phi$ is Euler totient function and $p$ runs through the prime divisors of $N$. Also, let

$$
g(N):=1+\frac{\mu_{0}(N)}{12}-\frac{\mu_{0,2}(N)}{4}-\frac{\mu_{0,3}(N)}{4}-\frac{c_{0}(N)}{2} .
$$

Then we have the following proposition which is taken from [28, Section 6.1, p. 93].

Proposition 2.3.1. We have $\operatorname{dim} S_{2}\left(\Gamma_{0}(N)\right)=g(N)$, and for $k \geq 4$ even,

$$
\begin{aligned}
\operatorname{dim} S_{k}\left(\Gamma_{0}(N)\right)=(k-1) & \cdot(g(N)-1)+\left(\frac{k}{2}-1\right) \cdot c(N) \\
+ & +\mu_{0,2}(N)\left\lfloor\frac{k}{4}\right\rfloor+\mu_{0,3}(N)\left\lfloor\frac{k}{3}\right\rfloor,
\end{aligned}
$$

where \lfloor\rfloor is the floor function. The dimension of the Eisenstein subspace is

$$
\operatorname{dim} E_{k}\left(\Gamma_{0}(N)\right)= \begin{cases}c_{0}(N)-1 & \text { if } k=2, \\ c_{0}(N) & \text { if } k \neq 2 .\end{cases}
$$


Example 2.3.2. Let $N=56$. We have $c(56)=8, \mu_{0}(N)=96, \mu_{0,2}(56)=\mu_{0,3}(56)=$ 0. Hence

$$
g(56)=1+\frac{96}{12}-\frac{8}{2}=5
$$

Thus by Propostion 2.3.1 we have $\operatorname{dim} S_{2}\left(\Gamma_{0}(56)\right)=5$ and $\operatorname{dim} E_{2}\left(\Gamma_{0}(56)\right)=7$.

Second, we state the non-trivial character case. The formulae are taken from $[28$, Section 6.3, p. 98-100]. Let $v_{p}(N)$ denote the largest $r \in \mathbb{N}_{0}$ such that $p^{r} \mid \mathbb{N}$ and let $c$ be the conductor of $\chi$. We set

$$
\lambda_{\left(p, N, v_{p}(c)\right)}= \begin{cases}p^{\frac{r}{2}}+p^{\frac{r}{2}-1} & \text { if } 2 \cdot v_{p}(c) \leq r \text { and } 2 \mid r, \\ 2 \cdot p^{\frac{r-1}{2}} & \text { if } 2 \cdot v_{p}(c) \leq r \text { and } 2 \nmid r, \\ 2 \cdot p^{r-v_{p}(c)} & \text { if } 2 \cdot v_{p}(c)>r .\end{cases}
$$

The rational numbers $\gamma_{3}$ and $\gamma_{4}$ are defined as follows

$$
\begin{aligned}
& \gamma_{3}(k)= \begin{cases}-1 / 3 & \text { if } k \equiv 2(\bmod 3), \\
0 & \text { if } k \equiv 1(\bmod 3), \\
1 / 3 & \text { if } k \equiv 0(\bmod 3),\end{cases} \\
& \gamma_{4}(k)= \begin{cases}-1 / 4 & \text { if } k \equiv 2(\bmod 4), \\
0 & \text { if } k i s \operatorname{odd}, \\
1 / 4 & \text { if } k \equiv 0(\bmod 4) .\end{cases}
\end{aligned}
$$


Let $\chi$ be a Dirichlet character of modulus $N$ for which $\chi(-1)=(-1)^{k}$. Then we have

$$
\begin{aligned}
& \operatorname{dim} S_{k}\left(\Gamma_{0}(N), \chi\right)-\operatorname{dim} M_{2-k}\left(\Gamma_{0}(N), \chi\right)=\frac{k-1}{12} \cdot \mu_{0}(N)-\frac{1}{2} \cdot \prod_{p \mid N} \lambda\left(p, N, v_{p}(c)\right) \\
& \quad+\gamma_{4}(k) \cdot \sum_{x \in A_{4}(N)} \chi(x)+\gamma_{3}(k) \cdot \sum_{x \in A_{3}(N)} \chi(x)
\end{aligned}
$$

where

$A_{4}(N)=\left\{x \in \mathbb{Z} / N \mathbb{Z}: x^{2}+1=0\right\}$ and $A_{3}(N)=\left\{x \in \mathbb{Z} / N \mathbb{Z}: x^{2}+x+1=0\right\}$.

To compute $\operatorname{dim} M_{k}\left(\Gamma_{0}(N), \chi\right)$ for $k \geq 2$, we use the fact that $\operatorname{dim} S_{k}\left(\Gamma_{0}(N), \chi\right)=0$ for $k \leq 0$. Then we have

$$
\begin{aligned}
\operatorname{dim} M_{k}\left(\Gamma_{0}(N), \chi\right)= & -\left(\operatorname{dim} S_{2-k}\left(\Gamma_{0}(N), \chi\right)-\operatorname{dim} M_{k}\left(\Gamma_{0}(N), \chi\right)\right) \\
= & -\left(\frac{1-k}{12} \cdot \mu_{0}(N)-\frac{1}{2} \cdot \prod_{p \mid N} \lambda\left(p, N, v_{p}(c)\right)\right. \\
& \left.+\gamma_{4}(2-k) \cdot \sum_{x \in A_{4}(N)} \chi(x)+\gamma_{3}(2-k) \cdot \sum_{x \in A_{3}(N)} \chi(x)\right),
\end{aligned}
$$

and

$$
\operatorname{dim} E_{k}\left(\Gamma_{0}(N), \chi\right)=\operatorname{dim} M_{k}\left(\Gamma_{0}(N), \chi\right)-\operatorname{dim} S_{k}\left(\Gamma_{0}(N), \chi\right) .
$$

Example 2.3.3. For $N=56, k=2, \chi_{3}(m)=\left(\frac{28}{m}\right), \chi_{5}(m)=\left(\frac{8}{m}\right)$ and $\chi_{6}(m)=\left(\frac{56}{m}\right)$, we have

$$
\sum_{x \in A_{4}(N)} \chi(x)=\sum_{x \in A_{3}(N)} \chi(x)=0 .
$$

Also, we have 


\begin{tabular}{|c|c|c|c|}
\hline$\chi$ & $\chi_{3}$ & $\chi_{5}$ & $\chi_{6}$ \\
\hline$\prod_{p \mid 56} \lambda\left(p, 56, v_{p}(c)\right)$ & 8 & 4 & 4 \\
\hline
\end{tabular}

Thus by (2.3.1)-(2.3.3) we obtain

\begin{tabular}{|c|c|c|c|}
\hline$\chi$ & $\operatorname{dim} M_{2}\left(\Gamma_{0}(56), \chi\right)$ & $\operatorname{dim} S_{2}\left(\Gamma_{0}(56), \chi\right)$ & $\operatorname{dim} E_{2}\left(\Gamma_{0}(56), \chi\right)$ \\
\hline$\chi_{3}$ & 12 & 4 & 8 \\
$\chi_{5}$ & 10 & 6 & 4 \\
$\chi_{6}$ & 10 & 6 & 4 \\
\hline
\end{tabular}




\section{Chapter 3}

\section{Representations by Quaternary}

\section{Quadratic Forms with Coefficients}

$1,2,7$ and 14

\subsection{Preliminaries}

We recall that, for $a_{1}, a_{2}, a_{3}, a_{4} \in \mathbb{N}$ and $n \in \mathbb{N}_{0}, N\left(a_{1}, a_{2}, a_{3}, a_{4} ; n\right)$ denotes the number of representations of $n$ by the quaternary form $a_{1} x_{1}^{2}+a_{2} x_{2}^{2}+a_{3} x_{3}^{2}+a_{4} x_{4}^{2}$, that is

$N\left(a_{1}, a_{2}, a_{3}, a_{4} ; n\right):=\operatorname{card}\left\{\left(x_{1}, x_{2}, x_{3}, x_{4}\right) \in \mathbb{Z}^{4} \mid n=a_{1} x_{1}^{2}+a_{2} x_{2}^{2}+a_{3} x_{3}^{2}+a_{4} x_{4}^{2}\right\}$.

We also have the simplifying assumptions

$$
a_{1} \leq a_{2} \leq a_{3} \leq a_{4}
$$


Table 3.1 .1

\begin{tabular}{|l|l|l|l|}
\hline$M_{2}\left(\Gamma_{0}(56), \chi_{0}\right)$ & $M_{2}\left(\Gamma_{0}(56), \chi_{3}\right)$ & $M_{2}\left(\Gamma_{0}(56), \chi_{5}\right)$ & $M_{2}\left(\Gamma_{0}(56), \chi_{6}\right)$ \\
\hline$(1,1,7,7)$ & $(1,1,1,7)$ & $(1,2,7,7)$ & $(1,1,2,7)$ \\
$(2,2,7,7)$ & $(1,2,2,7)$ & $(1,1,7,14)$ & $(2,2,2,7)$ \\
$(1,2,7,14)$ & $(1,7,7,7)$ & $(2,2,7,14)$ & $(2,7,7,7)$ \\
$(1,1,14,14)$ & $(1,1,2,14)$ & $(1,2,14,14)$ & $(1,1,1,14)$ \\
& $(2,7,7,14)$ & & $(1,2,2,14)$ \\
& $(1,7,14,14)$ & & $(1,7,7,14)$ \\
& & & $(2,7,14,14)$ \\
& & & $(1,14,14,14)$ \\
\hline
\end{tabular}

and

$$
\operatorname{gcd}\left(a_{1}, a_{2}, a_{3}, a_{4}\right)=1
$$

We also recall that $\chi_{0}$ denotes the trivial character. For $m \in \mathbb{Z}$ we define six characters by

$$
\begin{aligned}
& \chi_{1}(m)=\left(\frac{-7}{m}\right), \chi_{2}(m)=\left(\frac{-4}{m}\right), \chi_{3}(m)=\left(\frac{28}{m}\right), \\
& \chi_{4}(m)=\left(\frac{-8}{m}\right), \chi_{5}(m)=\left(\frac{8}{m}\right), \chi_{6}(m)=\left(\frac{56}{m}\right) .
\end{aligned}
$$

Under the simplifying assumptions (3.1.1) and (3.1.2) there are twenty-six quaternary quadratic forms $a_{1} x_{1}^{2}+a_{2} x_{2}^{2}+a_{3} x_{3}^{2}+a_{4} x_{4}^{2}$ for which $\varphi\left(q^{a_{1}}\right) \varphi\left(q^{a_{2}}\right) \varphi\left(q^{a_{3}}\right) \varphi\left(q^{a_{4}}\right) \in$ $M_{2}\left(\Gamma_{0}(56), \chi\right)$ where $\chi \in\left\{\chi_{0}, \chi_{3}, \chi_{5}, \chi_{6}\right\}$. Their coefficients $\left(a_{1}, a_{2}, a_{3}, a_{4}\right)$ are listed in Table 3.1.1.

Formulae for the four quaternary quadratic forms $(1,1,1,1),(1,1,2,2),(1,2,2,2)$, $(1,1,1,2)$ appeared in [1], [29]. In this chapter we determine formulae for the remaining twenty-two quaternary quadratic forms listed in Table 3.1.1. 
We use the following theorem to determine if an eta quotient $f(z)=\prod_{1 \leq \delta \mid N} \eta^{r_{\delta}}(\delta z)$ is in $M_{k}\left(\Gamma_{0}(N), \chi\right)$. See [11], [13, Corollary 2.3, p. 37], [14, Theorem 5.7, p. 99] and $[17]$.

Theorem 3.1.1. ( Ligozat) Let $N \in \mathbb{N}$ and let $f(z)=\prod_{1 \leq \delta \mid N} \eta^{r_{\delta}}(\delta z)$ be an eta quotient which satisfies the following conditions:

$$
\begin{aligned}
& \text { (L1) } \sum_{1 \leq \delta \mid N} \delta \cdot r_{\delta} \equiv 0(\bmod 24), \\
& \text { (L2) } \sum_{1 \leq \delta \mid N} \frac{N}{\delta} \cdot r_{\delta} \equiv 0(\bmod 24), \\
& \text { (L3) for each } d \mid N, \sum_{1 \leq \delta \mid N} \frac{\operatorname{gcd}(d, \delta)^{2} \cdot r_{\delta}}{\delta} \geq 0 .
\end{aligned}
$$

Then $f(z)$ is in $M_{k}\left(\Gamma_{0}(N), \chi\right)$, where $\chi$ is given by

$$
\chi(m)=\left(\frac{(-1)^{k} s}{m}\right)
$$

with weight

$$
k=\frac{1}{2} \sum_{1 \leq \delta \mid N} r_{\delta}
$$

and

$$
s=\prod_{1 \leq \delta \mid N} \delta^{r_{\delta}}
$$

In addition to the above conditions if $f(z)$ also satisfies the condition (L4) for each $d \mid N, \sum_{1 \leq \delta \mid N} \frac{\operatorname{gcd}(d, \delta)^{2} \cdot r_{\delta}}{\delta}>0$, then $f(z)$ is in $S_{k}\left(\Gamma_{0}(N), \chi\right)$. 


\subsection{The space $M_{2}\left(\Gamma_{0}(56), \chi_{0}\right)$}

In this section we determine formulae for $N\left(a_{1}, a_{2}, a_{3}, a_{4} ; n\right)$ for the quaternary quadratic forms listed in the first column of Table 3.1.1 in terms of $\sigma(n), \sigma(n / 2), \sigma(n / 4), \sigma(n / 7)$, $\sigma(n / 8), \sigma(n / 14), \sigma(n / 28), \sigma(n / 56)$, and $a_{k}(n)(1 \leq k \leq 5)$ defined by

$$
\begin{aligned}
& A_{1}(q)=\sum_{n=1}^{\infty} a_{1}(n) q^{n}=\eta(2 z) \eta(4 z) \eta(14 z) \eta(28 z), \\
& A_{2}(q)=\sum_{n=1}^{\infty} a_{2}(n) q^{n}=\frac{\eta^{3}(2 z) \eta^{3}(28 z)}{\eta(4 z) \eta(14 z)}, \\
& A_{3}(q)=\sum_{n=1}^{\infty} a_{3}(n) q^{n}=\frac{\eta(z) \eta^{3}(4 z) \eta(7 z) \eta^{3}(28 z)}{\eta(2 z) \eta(8 z) \eta(14 z) \eta(56 z)} \\
& A_{4}(q)=\sum_{n=1}^{\infty} a_{4}(n) q^{n}=\frac{\eta^{3}(2 z) \eta(8 z) \eta^{3}(14 z) \eta(56 z)}{\eta(z) \eta(4 z) \eta(7 z) \eta(28 z)} \\
& A_{5}(q)=\sum_{n=1}^{\infty} a_{5}(n) q^{n}=\frac{\eta^{4}(4 z) \eta^{4}(28 z)}{\eta(2 z) \eta(8 z) \eta(14 z) \eta(56 z)}
\end{aligned}
$$

There is no linear relationship among the $A_{k}(q), 1 \leq k \leq 5$. The first fifty-six values of $a_{k}(n)$, are given in Table 3.2.1.

Table 3.2.1

\begin{tabular}{r|rrrrr|r|rrrrr}
\hline$n$ & $a_{1}(n)$ & $a_{2}(n)$ & $a_{3}(n)$ & $a_{4}(n)$ & $a_{5}(n)$ & $n$ & $a_{1}(n)$ & $a_{2}(n)$ & $a_{3}(n)$ & $a_{4}(n)$ & $a_{5}(n)$ \\
\hline 1 & 0 & 0 & 1 & 0 & 0 & 29 & 0 & -2 & -2 & 2 & 0 \\
2 & 1 & 0 & -1 & 0 & 1 & 30 & 0 & 0 & 0 & 0 & 0 \\
3 & 0 & 1 & 0 & 1 & 0 & 31 & 0 & -2 & 0 & 2 & 0 \\
4 & -1 & 0 & -1 & 1 & 1 & 32 & 1 & 0 & 1 & -1 & -1 \\
5 & 0 & -3 & -2 & -1 & 0 & 33 & 0 & 0 & 0 & 0 & 0 \\
6 & -2 & 0 & 2 & 0 & -2 & 34 & 6 & 0 & -6 & 0 & 6 \\
7 & 0 & 1 & 1 & 0 & 0 & 35 & 0 & -1 & -2 & -1 & 0 \\
8 & 1 & 0 & 1 & -1 & -1 & 36 & -1 & 0 & -1 & 1 & 1 \\
9 & 0 & 2 & 1 & 0 & 0 & 37 & 0 & -2 & -2 & -2 & 0 \\
10 & 0 & 0 & 0 & 0 & 0 & 38 & 2 & 0 & -2 & 0 & 2 \\
11 & 0 & 2 & 0 & 0 & 0 & 39 & 0 & 0 & 4 & -2 & 0 \\
12 & 2 & 0 & 2 & -2 & -2 & 40 & 0 & 0 & 0 & 0 & 0 \\
13 & 0 & -1 & -2 & 1 & 0 & 41 & 0 & -2 & 2 & -2 & 0 \\
14 & 1 & 0 & -1 & 0 & 1 & 42 & -2 & 0 & 2 & 0 & -2 \\
15 & 0 & -4 & -4 & -2 & 0 & 43 & 0 & 6 & 8 & 0 & 0 \\
16 & -1 & 0 & -1 & 1 & 1 & 44 & 0 & 0 & 0 & 0 & 0 \\
17 & 0 & 2 & 2 & -2 & 0 & 45 & 0 & 1 & -2 & -1 & 0
\end{tabular}




\begin{tabular}{r|rrrrr|r|rrrrr}
18 & 1 & 0 & -1 & 0 & 1 & 46 & 0 & 0 & 0 & 0 & 0 \\
19 & 0 & -5 & 0 & -1 & 0 & 47 & 0 & 2 & -8 & 2 & 0 \\
20 & 0 & 0 & 0 & 0 & 0 & 48 & 2 & 0 & 2 & -2 & -2 \\
21 & 0 & 1 & 0 & 1 & 0 & 49 & 0 & 0 & 1 & 0 & 0 \\
22 & 0 & 0 & 0 & 0 & 0 & 50 & -5 & 0 & 5 & 0 & -5 \\
23 & 0 & 4 & 4 & 2 & 0 & 51 & 0 & -2 & -8 & 2 & 0 \\
24 & -2 & 0 & -2 & 2 & 2 & 52 & 4 & 0 & 4 & -4 & -4 \\
25 & 0 & 6 & 3 & 4 & 0 & 53 & 0 & -8 & -2 & -4 & 0 \\
26 & -4 & 0 & 4 & 0 & -4 & 54 & 4 & 0 & -4 & 0 & 4 \\
27 & 0 & -2 & 0 & -2 & 0 & 55 & 0 & 4 & 0 & 0 & 0 \\
28 & -1 & 0 & -1 & 1 & 1 & 56 & 1 & 0 & 1 & -1 & -1 \\
\hline
\end{tabular}

Theorem 3.2.1. Let $\left(a_{1}, a_{2}, a_{3}, a_{4}\right)$ be as in the first column of Table 3.1.1. Then $\varphi\left(q^{a_{1}}\right) \varphi\left(q^{a_{2}}\right) \varphi\left(q^{a_{3}}\right) \varphi\left(q^{a_{4}}\right) \in M_{2}\left(\Gamma_{0}(56), \chi_{0}\right)$.

Proof. Appealing to (2.1.9) for each quadratic form, we then check conditions (L1), (L2) and (L3) of Theorem 3.1 .1 for each form. We have $N=56$. First we consider $(1,1,7,7)$

$$
\varphi^{2}(q) \varphi^{2}\left(q^{7}\right)=\frac{\eta^{10}(2 z) \eta^{10}(14 z)}{\eta^{4}(z) \eta^{4}(4 z) \eta^{4}(7 z) \eta^{4}(28 z)}
$$

Then we have

Table 3.2.2(a)

\begin{tabular}{r|rrrrrr}
\hline$\delta$ & 1 & 2 & 4 & 7 & 14 & 28 \\
\hline$r_{\delta}$ & -4 & 10 & -4 & -4 & 10 & -4 \\
\hline
\end{tabular}

It can be seen from Table 3.2.2(a) that conditions $(L 1)$ and $(L 2)$ are satisfied.

Table 3.2.2(b)

\begin{tabular}{r|rrrrrrrr}
\hline$d \mid 56$ & 1 & 2 & 4 & 7 & 8 & 14 & 28 & 56 \\
\hline$\sum_{1 \leq \delta \mid 56} \frac{\operatorname{gcd}(d, \delta)^{2} \cdot r_{\delta}}{\delta}$ & 0 & $96 / 7$ & 0 & 0 & 0 & 96 & 0 & 0 \\
\hline
\end{tabular}


From Table 3.2.2(b) the condition (L3) is also satisfied. Thus $\varphi^{2}(q) \varphi^{2}\left(q^{7}\right)$ $\in M_{2}\left(\Gamma_{0}(56), \chi_{0}\right)$. Second we consider $(2,2,7,7)$

$$
\varphi^{2}\left(q^{2}\right) \varphi^{2}\left(q^{7}\right)=\frac{\eta^{10}(4 z) \eta^{10}(14 z)}{\eta^{4}(2 z) \eta^{4}(7 z) \eta^{4}(8 z) \eta^{4}(28 z)}
$$

Then we have

Table 3.2.3(a)

\begin{tabular}{r|rrrrrr}
\hline$\delta$ & 2 & 4 & 7 & 8 & 14 & 28 \\
\hline$r_{\delta}$ & -4 & 10 & -4 & -4 & 10 & -4 \\
\hline
\end{tabular}

It can be seen from Table 3.2.3(a) that conditions $(L 1)$ and $(L 2)$ are satisfied.

Table 3.2.3(b)

\begin{tabular}{r|rrrrrrrr}
\hline$d \mid 56$ & 1 & 2 & 4 & 7 & 8 & 14 & 28 & 56 \\
\hline$\sum_{1 \leq \delta \mid 56} \frac{\operatorname{gcd}(d, \delta)^{2} \cdot r_{\delta}}{\delta}$ & 0 & $12 / 7$ & 24 & 0 & 0 & 84 & 24 & 0 \\
\hline
\end{tabular}

From Table 3.2.3(b) the condition (L3) is also satisfied. Thus $\varphi^{2}\left(q^{2}\right) \varphi^{2}\left(q^{7}\right)$ $\in M_{2}\left(\Gamma_{0}(56), \chi_{0}\right)$. Third we consider $(1,2,7,14)$

$$
\varphi(q) \varphi\left(q^{2}\right) \varphi\left(q^{7}\right) \varphi\left(q^{14}\right)=\frac{\eta^{3}(2 z) \eta^{3}(4 z) \eta^{3}(14 z) \eta^{3}(28 z)}{\eta^{2}(z) \eta^{2}(7 z) \eta^{2}(8 z) \eta^{2}(56 z)}
$$

Then we have

Table 3.2.4(a)

\begin{tabular}{r|rrrrrrrr}
\hline$\delta$ & 1 & 2 & 4 & 7 & 8 & 14 & 28 & 56 \\
\hline$r_{\delta}$ & -2 & 3 & 3 & -2 & -2 & 3 & 3 & -2 \\
\hline
\end{tabular}

It can be seen from Table 3.2.4(a) that conditions (L1) and (L2) are satisfied. 
Table 3.2.4(b)

\begin{tabular}{r|rrrrrrrr}
\hline$d \mid 56$ & 1 & 2 & 4 & 7 & 8 & 14 & 28 & 56 \\
\hline$\sum_{1 \leq \delta \mid 56} \frac{\operatorname{gcd}(d, \delta)^{2} \cdot r_{\delta}}{\delta}$ & 0 & $48 / 7$ & $96 / 7$ & 0 & 0 & 48 & 96 & 0 \\
\hline
\end{tabular}

From Table 3.2.4(b) the condition (L3) is also satisfied. Thus $\varphi(q) \varphi\left(q^{2}\right) \varphi\left(q^{7}\right) \varphi\left(q^{14}\right) \in$ $M_{2}\left(\Gamma_{0}(56), \chi_{0}\right)$. Fourth we consider $(1,1,14,14)$

$$
\varphi^{2}(q) \varphi^{2}\left(q^{14}\right)=\frac{\eta^{10}(2 z) \eta^{10}(28 z)}{\eta^{4}(z) \eta^{4}(4 z) \eta^{4}(14 z) \eta^{4}(56 z)}
$$

Then we have

Table 3.2.5(a)

\begin{tabular}{r|rrrrrr}
\hline$\delta$ & 1 & 2 & 4 & 14 & 28 & 56 \\
\hline$r_{\delta}$ & -4 & 10 & -4 & -4 & 10 & -4 \\
\hline
\end{tabular}

It can be seen from Table 3.2.5(a) that conditions (L1) and (L2) are satisfied.

Table 3.2.5(b)

\begin{tabular}{r|rrrrrrrr}
\hline$d \mid 56$ & 1 & 2 & 4 & 7 & 8 & 14 & 28 & 56 \\
\hline$\sum_{1 \leq \delta \mid 56} \frac{\operatorname{gcd}(d, \delta)^{2} \cdot r_{\delta}}{\delta}$ & 0 & 12 & $24 / 7$ & 0 & 0 & 12 & 168 & 0 \\
\hline
\end{tabular}

From Table 3.2.5(b) the condition $(L 3)$ is also satisfied. Thus $\varphi^{2}(q) \varphi^{2}\left(q^{14}\right)$ $\in M_{2}\left(\Gamma_{0}(56), \chi_{0}\right)$.

Theorem 3.2.2. $A_{k}(q)(1 \leq k \leq 5)$ given by $(3.2 .1)-(3.2 .5)$ are in $S_{2}\left(\Gamma_{0}(56), \chi_{0}\right)$.

Proof. We will check conditions (L1), (L2) and (L4) of Theorem 3.1.1. We have $N=56$. First we consider

$$
A_{1}(q)=\eta(2 z) \eta(4 z) \eta(14 z) \eta(28 z)
$$

Then we have 
Table 3.2.6(a)

\begin{tabular}{r|rrrr}
\hline$\delta$ & 2 & 4 & 14 & 28 \\
\hline$r_{\delta}$ & 1 & 1 & 1 & 1 \\
\hline
\end{tabular}

It can be seen from Table 3.2.6(a) that conditions (L1) and (L2) are satisfied.

Table 3.2.6(b)

\begin{tabular}{r|rrrrrrrr}
\hline$d \mid 56$ & 1 & 2 & 4 & 7 & 8 & 14 & 28 & 56 \\
\hline$\sum_{1 \leq \delta \mid 56} \frac{\operatorname{gcd}(d, \delta)^{2} \cdot r_{\delta}}{\delta}$ & $6 / 7$ & $24 / 7$ & $48 / 7$ & 6 & $48 / 7$ & 24 & 48 & 48 \\
\hline
\end{tabular}

From Table 3.2.6(b) the condition $(L 4)$ is also satisfied. Thus $A_{1}(q) \in S_{2}\left(\Gamma_{0}(56), \chi_{0}\right)$. Then

$$
A_{2}(q)=\frac{\eta^{3}(2 z) \eta^{3}(28 z)}{\eta(4 z) \eta(14 z)}
$$

Then we have

Table 3.2.7(a)

\begin{tabular}{r|rrrr}
\hline$\delta$ & 2 & 4 & 14 & 28 \\
\hline$r_{\delta}$ & 3 & -1 & -1 & 3 \\
\hline
\end{tabular}

It can be seen from Table 3.2.7(a) that conditions (L1) and (L2) are satisfied.

\begin{tabular}{r|rrrrrrrr}
\multicolumn{1}{c}{ Table 3.2.7(b) } \\
\hline$d \mid 56$ & 1 & 2 & 4 & 7 & 8 & 14 & 28 & 56 \\
\hline$\sum_{1 \leq \delta \mid 56} \frac{\operatorname{gcd}(d, \delta)^{2} \cdot r_{\delta}}{\delta}$ & $9 / 7$ & $36 / 7$ & $24 / 7$ & 3 & $24 / 7$ & 12 & 72 & 72 \\
\hline
\end{tabular}

From Table 3.2.7(b) the condition ( $L 4)$ is also satisfied. thus $A_{2}(q) \in S_{2}\left(\Gamma_{0}(56), \chi_{0}\right)$. Then

$$
A_{3}(q)=\frac{\eta(z) \eta^{3}(4 z) \eta(7 z) \eta^{3}(28 z)}{\eta(2 z) \eta(8 z) \eta(14 z) \eta(56 z)}
$$

Then we have 
Table 3.2.8(a)

\begin{tabular}{r|rrrrrrrr}
\hline$\delta$ & 1 & 2 & 4 & 7 & 8 & 14 & 28 & 56 \\
\hline$r_{\delta}$ & 1 & -1 & 3 & 1 & -1 & -1 & 3 & -1 \\
\hline
\end{tabular}

It can be seen from Table 3.2.8(a) that conditions (L1) and (L2) are satisfied.

Table 3.2.8(b)

\begin{tabular}{r|rrrrrrrr}
\hline$d \mid 56$ & 1 & 2 & 4 & 7 & 8 & 14 & 28 & 56 \\
\hline$\sum_{1 \leq \delta \mid 56} \frac{\operatorname{gcd}(d, \delta)^{2} \cdot r_{\delta}}{\delta}$ & $9 / 7$ & $12 / 7$ & $72 / 7$ & 9 & $24 / 7$ & 12 & 72 & 24 \\
\hline
\end{tabular}

From Table 3.2.8(b) the condition $(L 4)$ is also satisfied. Thus $A_{3}(q) \in S_{2}\left(\Gamma_{0}(56), \chi_{0}\right)$. Then

$$
A_{4}(q)=\frac{\eta^{3}(2 z) \eta(8 z) \eta^{3}(14 z) \eta(56 z)}{\eta(z) \eta(4 z) \eta(7 z) \eta(28 z)}
$$

Then we have

Table 3.2.9(a)

\begin{tabular}{r|rrrrrrrr}
\hline$\delta$ & 1 & 2 & 4 & 7 & 8 & 14 & 28 & 56 \\
\hline$r_{\delta}$ & -1 & 3 & -1 & -1 & 1 & 3 & -1 & 1 \\
\hline
\end{tabular}

It can be seen from Table 3.2.9(a) that conditions (L1) and (L2) are satisfied.

\begin{tabular}{r|rrrrrrrr}
\multicolumn{1}{c}{ Table 3.2.9(b) } \\
\hline$d \mid 56$ & 1 & 2 & 4 & 7 & 8 & 14 & 28 & 56 \\
\hline$\sum_{1 \leq \delta \mid 56} \frac{\operatorname{gcd}(d, \delta)^{2} \cdot r_{\delta}}{\delta}$ & $3 / 7$ & $36 / 7$ & $24 / 7$ & 3 & $72 / 7$ & 36 & 24 & 72 \\
\hline
\end{tabular}

From Table 3.2.9(b) the condition $(L 4)$ is also satisfied. Thus $A_{4}(q) \in S_{2}\left(\Gamma_{0}(56), \chi_{0}\right)$. Then

$$
A_{5}(q)=\frac{\eta^{4}(4 z) \eta^{4}(28 z)}{\eta(2 z) \eta(8 z) \eta(14 z) \eta(56 z)}
$$

Then we have 
Table 3.2.10(a)

\begin{tabular}{r|rrrrrr}
\hline$\delta$ & 2 & 4 & 8 & 14 & 28 & 56 \\
\hline$r_{\delta}$ & -1 & 4 & -1 & -1 & 4 & -1 \\
\hline
\end{tabular}

It can be seen from Table 3.2.10(a) that conditions (L1) and (L2) are satisfied.

Table 3.2.10(b)

\begin{tabular}{r|rrrrrrrr}
\hline$d \mid 56$ & 1 & 2 & 4 & 7 & 8 & 14 & 28 & 56 \\
\hline$\sum_{1 \leq \delta \mid 56} \frac{\operatorname{gcd}(d, \delta)^{2} \cdot r_{\delta}}{\delta}$ & $3 / 7$ & $12 / 7$ & $96 / 7$ & 3 & $48 / 7$ & 12 & 96 & 48 \\
\hline
\end{tabular}

From Table 3.2.10(b) the condition $(L 4)$ is also satisfied. Thus $A_{5}(q)$ $\in S_{2}\left(\Gamma_{0}(56), \chi_{0}\right)$

Theorem 3.2.3. (a) $\left\{A_{1}(q), \ldots, A_{5}(q)\right\}$ constitute a basis for $S_{2}\left(\Gamma_{0}(56), \chi_{0}\right)$.

(b) $L(q)-t L\left(q^{t}\right)(t=2,4,7,8,14,28,56)$ constitute a basis for $E_{2}\left(\Gamma_{0}(56), \chi_{0}\right)$.

(c) $L(q)-t L\left(q^{t}\right)(t=2,4,7,8,14,28,56)$ together with $A_{k}(q)(1 \leq k \leq 5)$ constitute a basis for $M_{2}\left(\Gamma_{0}(56), \chi_{0}\right)$.

Proof. (a) By Theorem 3.2.2, $A_{k}(q)(1 \leq k \leq 5) \in S_{2}\left(\Gamma_{0}(56), \chi_{0}\right)$. There is no linear relationship among them. By Example 2.3.2, we have $\operatorname{dim} S_{2}\left(\Gamma_{0}(56), \chi_{0}\right)=5$. Thus $A_{k}(q)(1 \leq k \leq 5)$ constitute a basis for $S_{2}\left(\Gamma_{0}(56), \chi_{0}\right)$.

(b) By Example 2.3.2, we have $\operatorname{dim} E_{2}\left(\Gamma_{0}(56), \chi_{0}\right)=7$. By Theorem 2.2.3, $L(q)-$ $t L\left(q^{t}\right)(t=2,4,7,8,14,28,56)$ constitute a basis for $E_{2}\left(\Gamma_{0}(56), \chi_{0}\right)$.

(c) It follows from (a), (b) and (2.1.1) that the dimension of $M_{2}\left(\Gamma_{0}(56), \chi_{0}\right)$ is 12 and therefore $L(q)-t L\left(q^{t}\right)(t=2,4,7,8,14,28,56)$ together with $A_{k}(q)(1 \leq k \leq 5)$ constitute a basis for $M_{2}\left(\Gamma_{0}(56), \chi_{0}\right)$.

\section{Theorem 3.2.4.}

$$
\begin{aligned}
\text { (a) } \varphi^{2}(q) \varphi^{2}\left(q^{7}\right)= & \frac{4}{3} L(q)-\frac{8}{3} L\left(q^{2}\right)+\frac{16}{3} L\left(q^{4}\right)-\frac{28}{3} L\left(q^{7}\right)+\frac{56}{3} L\left(q^{14}\right) \\
& -\frac{112}{3} L\left(q^{28}\right)+\frac{8}{3} A_{3}(q)-\frac{16}{3} A_{4}(q)+\frac{16}{3} A_{5}(q)
\end{aligned}
$$


(b) $\varphi^{2}\left(q^{2}\right) \varphi^{2}\left(q^{7}\right)=\frac{2}{3} L(q)-\frac{2}{3} L\left(q^{2}\right)-\frac{4}{3} L\left(q^{4}\right)-\frac{14}{3} L\left(q^{7}\right)+\frac{16}{3} L\left(q^{8}\right)$

$$
\begin{aligned}
& +\frac{14}{3} L\left(q^{14}\right)+\frac{28}{3} L\left(q^{28}\right)-\frac{112}{3} L\left(q^{56}\right)-\frac{10}{3} A_{1}(q)+4 A_{2}(q) \\
& -\frac{2}{3} A_{3}(q)-\frac{20}{3} A_{4}(q)+\frac{16}{3} A_{5}(q),
\end{aligned}
$$

(c) $\varphi(q) \varphi\left(q^{2}\right) \varphi\left(q^{7}\right) \varphi\left(q^{14}\right)=\frac{2}{3} L(q)-\frac{2}{3} L\left(q^{2}\right)-\frac{4}{3} L\left(q^{4}\right)-\frac{14}{3} L\left(q^{7}\right)$

$$
\begin{aligned}
& +\frac{16}{3} L\left(q^{8}\right)+\frac{14}{3} L\left(q^{14}\right)+\frac{28}{3} L\left(q^{28}\right)-\frac{112}{3} L\left(q^{56}\right) \\
& +\frac{2}{3} A_{1}(q)+\frac{4}{3} A_{3}(q)+\frac{4}{3} A_{4}(q)+\frac{4}{3} A_{5}(q)
\end{aligned}
$$

(d) $\varphi^{2}(q) \varphi^{2}\left(q^{14}\right)=\frac{2}{3} L(q)-\frac{2}{3} L\left(q^{2}\right)-\frac{4}{3} L\left(q^{4}\right)-\frac{14}{3} L\left(q^{7}\right)+\frac{16}{3} L\left(q^{8}\right)$

$$
\begin{aligned}
& +\frac{14}{3} L\left(q^{14}\right)+\frac{28}{3} L\left(q^{28}\right)-\frac{112}{3} L\left(q^{56}\right)+\frac{2}{3} A_{1}(q)-4 A_{2}(q) \\
& +\frac{10}{3} A_{3}(q)+\frac{4}{3} A_{4}(q)+\frac{16}{3} A_{5}(q) .
\end{aligned}
$$

Proof. Let $\left(a_{1}, a_{2}, a_{3}, a_{4}\right)$ be one of the quadratic forms listed in the first column of Table 3.1.1. By Theorem 3.2.1 and Theorem 3.2.3 (c), $\varphi\left(q^{a_{1}}\right) \varphi\left(q^{a_{2}}\right) \varphi\left(q^{a_{3}}\right) \varphi\left(q^{a_{4}}\right)$ must be a linear combinations of $L(q)-t L\left(q^{t}\right)(t=2,4,7,8,14,28,56)$ and $A_{k}(q)$ $(1 \leq k \leq 5)$, namely

$$
\begin{aligned}
\varphi\left(q^{a_{1}}\right) & \varphi\left(q^{a_{2}}\right) \varphi\left(q^{a_{3}}\right) \varphi\left(q^{a_{4}}\right)=x_{1}\left(L(q)-2 L\left(q^{2}\right)\right)+x_{2}\left(L(q)-4 L\left(q^{4}\right)\right) \\
& +x_{3}\left(L(q)-7 L\left(q^{7}\right)\right)+x_{4}\left(L(q)-8 L\left(q^{8}\right)\right)+x_{5}\left(L(q)-14 L\left(q^{14}\right)\right) \\
& +x_{6}\left(L(q)-28 L\left(q^{28}\right)\right)+x_{7}\left(L(q)-56 L\left(q^{56}\right)\right)+y_{1} A_{1}(q)+y_{2} A_{2}(q) \\
& +y_{3} A_{3}(q)+y_{4} A_{4}(q)+y_{5} A_{5}(q) .
\end{aligned}
$$

We equate the first 60 coefficients of $q^{n}$ on both sides of the equation above to obtain a system of linear equations with the unknowns $x_{1}, x_{2}, x_{3}, x_{4}, x_{5}, x_{6}, x_{7}, y_{1}, y_{2}, y_{3}, y_{4}, y_{5}$. Then, using MAPLE we solve the system to find the asserted coefficients. 
We now give an explicit formulae for $N\left(a_{1}, a_{2}, a_{3}, a_{4} ; n\right)$ for the quadratic forms $\left(a_{1}, a_{2}, a_{3}, a_{4}\right)$ in Theorem 3.2.4 in terms of $\sigma(n / d)(d=1,2,4,7,14,28,56)$ and $a_{k}(n)$ $(1 \leq k \leq 5)$.

Theorem 3.2.5. Let $n \in \mathbb{N}$. Then

(a) $N(1,1,7,7 ; n)=\frac{4}{3} \sigma(n)-\frac{8}{3} \sigma(n / 2)+\frac{16}{3} \sigma(n / 4)-\frac{28}{3} \sigma(n / 7)+\frac{56}{3} \sigma(n / 14)$

$$
-\frac{112}{3} \sigma(n / 28)+\frac{8}{3} a_{3}(n)-\frac{16}{3} a_{4}(n)+\frac{16}{3} a_{5}(n),
$$

(b) $N(2,2,7,7 ; n)=\frac{2}{3} \sigma(n)-\frac{2}{3} \sigma(n / 2)-\frac{4}{3} \sigma(n / 4)-\frac{14}{3} \sigma(n / 7)+\frac{16}{3} \sigma(n / 8)$

$$
\begin{aligned}
& +\frac{14}{3} \sigma(n / 14)+\frac{28}{3} \sigma(n / 28)-\frac{112}{3} \sigma(n / 56)-\frac{10}{3} a_{1}(n) \\
& +4 a_{2}(n)-\frac{2}{3} a_{3}(n)-\frac{20}{3} a_{4}(n)+\frac{16}{3} a_{5}(n),
\end{aligned}
$$

(c) $N(1,2,7,14 ; n)=\frac{2}{3} \sigma(n)-\frac{2}{3} \sigma(n / 2)-\frac{4}{3} \sigma(n / 4)-\frac{14}{3} \sigma(n / 7)+\frac{16}{3} \sigma(n / 8)$

$$
\begin{aligned}
& +\frac{14}{3} \sigma(n / 14)+\frac{28}{3} \sigma(n / 28)-\frac{112}{3} \sigma(n / 56)+\frac{2}{3} a_{1}(n) \\
& +\frac{4}{3} a_{3}(n)+\frac{4}{3} a_{4}(n)+\frac{4}{3} a_{5}(n)
\end{aligned}
$$

(d) $N(1,1,14,14 ; n)=\frac{2}{3} \sigma(n)-\frac{2}{3} \sigma(n / 2)-\frac{4}{3} \sigma(n / 4)-\frac{14}{3} \sigma(n / 7)+\frac{16}{3} \sigma(n / 8)$

$$
\begin{aligned}
& +\frac{14}{3} \sigma(n / 14)+\frac{28}{3} \sigma(n / 28)-\frac{112}{3} \sigma(n / 56)+\frac{2}{3} a_{1}(n) \\
& -4 a_{2}(n)+\frac{10}{3} a_{3}(n)+\frac{4}{3} a_{4}(n)+\frac{16}{3} a_{5}(n) .
\end{aligned}
$$

Proof. From (2.1.6), (2.2.3) and Theorem 3.2.4, we obtain

$$
\text { (a) } \begin{aligned}
& \sum_{n=0}^{\infty} N(1,1,7,7 ; n) q^{n}=\varphi^{2}(q) \varphi^{2}\left(q^{7}\right) \\
&= 1+\sum_{n=1}^{\infty}\left(\frac{4}{3} \sigma(n)-\frac{8}{3} \sigma(n / 2)+\frac{16}{3} \sigma(n / 4)-\frac{28}{3} \sigma(n / 7)+\frac{56}{3} \sigma(n / 14)\right. \\
&\left.-\frac{112}{3} \sigma(n / 28)+\frac{8}{3} a_{3}(n)-\frac{16}{3} a_{4}(n)+\frac{16}{3} a_{5}(n)\right) q^{n}
\end{aligned}
$$


(b) $\sum_{n=0}^{\infty} N(2,2,7,7 ; n) q^{n}=\varphi^{2}\left(q^{2}\right) \varphi^{2}\left(q^{7}\right)$

$$
\begin{aligned}
= & 1+\sum_{n=1}^{\infty}\left(\frac{2}{3} \sigma(n)-\frac{2}{3} \sigma(n / 2)-\frac{4}{3} \sigma(n / 4)-\frac{14}{3} \sigma(n / 7)+\frac{16}{3} \sigma(n / 8)+\frac{14}{3} \sigma(n / 14)\right. \\
& +\frac{28}{3} \sigma(n / 28)-\frac{112}{3} \sigma(n / 56)-\frac{10}{3} a_{1}(n)+4 a_{2}(n)-\frac{2}{3} a_{3}(n)-\frac{20}{3} a_{4}(n) \\
& \left.+\frac{16}{3} a_{5}(n)\right) q^{n},
\end{aligned}
$$

(c) $\sum_{n=0}^{\infty} N(1,2,7,14 ; n) q^{n}=\varphi(q) \varphi\left(q^{2}\right) \varphi\left(q^{7}\right) \varphi\left(q^{14}\right)$

$$
=1+\sum_{n=1}^{\infty}\left(\frac{2}{3} \sigma(n)-\frac{2}{3} \sigma(n / 2)-\frac{4}{3} \sigma(n / 4)-\frac{14}{3} \sigma(n / 7)+\frac{16}{3} \sigma(n / 8)\right.
$$$$
+\frac{14}{3} \sigma(n / 14)+\frac{28}{3} \sigma(n / 28)-\frac{112}{3} \sigma(n / 56)+\frac{2}{3} a_{1}(n)+\frac{4}{3} a_{3}(n)+\frac{4}{3} a_{4}(n)
$$$$
\left.+\frac{4}{3} a_{5}(n)\right) q^{n}
$$

(d) $\sum_{n=0}^{\infty} N(1,1,14,14 ; n) q^{n}=\varphi^{2}(q) \varphi^{2}\left(q^{14}\right)$

$$
\begin{aligned}
= & 1+\sum_{n=1}^{\infty}\left(\frac{2}{3} \sigma(n)-\frac{2}{3} \sigma(n / 2)-\frac{4}{3} \sigma(n / 4)-\frac{14}{3} \sigma(n / 7)+\frac{16}{3} \sigma(n / 8)+\frac{14}{3} \sigma(n / 14)\right. \\
& +\frac{28}{3} \sigma(n / 28)-\frac{112}{3} \sigma(n / 56)+\frac{2}{3} a_{1}(n)-4 a_{2}(n)+\frac{10}{3} a_{3}(n)+\frac{4}{3} a_{4}(n) \\
& \left.+\frac{16}{3} a_{5}(n)\right) q^{n} .
\end{aligned}
$$

Equating the coefficients of $q^{n}$ on both sides of equations (a)-(d) yields the results.

For $\left(a_{1}, a_{2}, a_{3}, a_{4}\right)=(1,1,7,7),(1,1,14,14),(2,2,7,7),(1,2,7,14)$, the values of $N\left(a_{1}, a_{2}, a_{3}, a_{4} ; n\right)$ for $1 \leq n \leq 20$ are given in Table 3.2.11. One can verify them by using Table 3.2.1.

Table 3.2 .11

\begin{tabular}{l|rrrrrrrrrrrrrrrrrrrr}
\hline \multicolumn{1}{c|}{$n$} & 1 & 2 & 3 & 4 & 5 & 6 & 7 & 8 & 9 & 10 & 11 & 12 & 13 & 14 & 15 & 16 & 17 & 18 & 19 & 20 \\
\hline$N(1,1,7,7 ; n)$ & 4 & 4 & 0 & 4 & 8 & 0 & 4 & 20 & 20 & 8 & 16 & 32 & 8 & 4 & 32 & 36 & 40 & 20 & 32 & 40 \\
$N(2,2,7,7 ; n)$ & 0 & 4 & 0 & 4 & 0 & 0 & 4 & 4 & 16 & 8 & 16 & 0 & 0 & 4 & 16 & 20 & 32 & 20 & 0 & 8 \\
$N(1,2,7,14 ; n)$ & 2 & 2 & 4 & 2 & 0 & 4 & 2 & 6 & 10 & 8 & 8 & 4 & 8 & 2 & 8 & 18 & 12 & 18 & 12 & 8
\end{tabular}




\begin{tabular}{l|llllllllllllllllllll}
$N(1,1,14,14 ; n)$ & 4 & 4 & 0 & 4 & 8 & 0 & 0 & 4 & 4 & 8 & 0 & 0 & 8 & 4 & 16 & 20 & 8 & 20 & 32 & 8 \\
\hline
\end{tabular}

For example, using Table 3.2.1, we obtain

$$
\begin{aligned}
N(2,2,7,7 ; 16)= & \frac{2}{3} \sigma(16)-\frac{2}{3} \sigma(8)-\frac{4}{3} \sigma(4)-\frac{14}{3} \sigma(16 / 7)+\frac{16}{3} \sigma(2) \\
& +\frac{14}{3} \sigma(16 / 14)+\frac{28}{3} \sigma(16 / 28)-\frac{112}{3} \sigma(16 / 56)-\frac{10}{3} a_{1}(16) \\
& +4 a_{2}(16)-\frac{2}{3} a_{3}(16)-\frac{20}{3} a_{4}(16)+\frac{16}{3} a_{5}(16) \\
= & \frac{2}{3}(31)-\frac{2}{3}(15)-\frac{4}{3}(7)+\frac{16}{3}(3)-\frac{10}{3}(-1)-\frac{2}{3}(-1)-\frac{20}{3}+\frac{16}{3} \\
= & 20
\end{aligned}
$$

which agrees with the value of $N(2,2,7,7 ; 16)$ in Table 3.2.11.

\subsection{The space $M_{2}\left(\Gamma_{0}(56), \chi_{3}\right)$}

Let $\chi_{0}$ be the trivial character and $\chi_{1}, \chi_{2}, \chi_{3}$ as in (3.1.3). We define the Eisenstein series

$$
\begin{aligned}
& E_{2, \chi_{3}, \chi_{0}}(q)=\sum_{n=1}^{\infty} \sigma_{\left(\chi_{3}, \chi_{0}\right)}(n) q^{n}, \\
& E_{2, \chi_{0}, \chi_{3}}(q)=-4+\sum_{n=1}^{\infty} \sigma_{\left(\chi_{0}, \chi_{3}\right)}(n) q^{n}, \\
& E_{2, \chi_{1}, \chi_{2}}(q)=\sum_{n=1}^{\infty} \sigma_{\left(\chi_{1}, \chi_{2}\right)}(n) q^{n}, \\
& E_{2, \chi_{2}, \chi_{1}}(q)=\sum_{n=1}^{\infty} \sigma_{\left(\chi_{2}, \chi_{1}\right)}(n) q^{n} .
\end{aligned}
$$

We determine $N\left(a_{1}, a_{2}, a_{3}, a_{4} ; n\right)$ for the six quaternary quadratic forms listed in the second column of Table 3.1.1 in terms of $\sigma_{(\chi, \psi)}(n)$, where $\chi, \psi \in\left\{\chi_{0}, \chi_{1}, \chi_{2}, \chi_{3}\right\}$, and 
$b_{k}(n)(1 \leq k \leq 4)$ defined by

$$
\begin{aligned}
& B_{1}(q)=\sum_{n=1}^{\infty} b_{1}(n) q^{n}=\frac{\eta^{2}(2 z) \eta^{3}(7 z)}{\eta(z)}, \\
& B_{2}(q)=\sum_{n=1}^{\infty} b_{2}(n) q^{n}=\frac{\eta^{3}(8 z) \eta^{2}(28 z)}{\eta(56 z)}, \\
& B_{3}(q)=\sum_{n=1}^{\infty} b_{3}(n) q^{n}=\frac{\eta^{2}(4 z) \eta^{3}(56 z)}{\eta(8 z)}, \\
& B_{4}(q)=\sum_{n=1}^{\infty} b_{4}(n) q^{n}=\frac{\eta^{3}(z) \eta^{2}(14 z)}{\eta(7 z)} .
\end{aligned}
$$

There is no linear relationship among the $B_{k}(q), 1 \leq k \leq 4$. The first fifty-six values of $b_{k}(n), 1 \leq k \leq 4$, are given in Table 3.3.1.

Table 3.3.1

\begin{tabular}{r|rrrr|r|rrrr}
\hline$n$ & $b_{1}(n)$ & $b_{2}(n)$ & $b_{3}(n)$ & $b_{4}(n)$ & $n$ & $b_{1}(n)$ & $b_{2}(n)$ & $b_{3}(n)$ & $b_{4}(n)$ \\
\hline 1 & 1 & 1 & 0 & 1 & 29 & -2 & -2 & 0 & -2 \\
2 & 1 & 0 & 0 & -3 & 30 & 0 & 0 & 0 & 0 \\
3 & 0 & 0 & 0 & 0 & 31 & 0 & 0 & 0 & 0 \\
4 & 1 & 0 & 0 & 5 & 32 & 5 & 0 & 0 & 9 \\
5 & 0 & 0 & 0 & 0 & 33 & 0 & 0 & 0 & 0 \\
6 & 0 & 0 & 0 & 0 & 34 & 0 & 0 & 0 & 0 \\
7 & 1 & 0 & 1 & -7 & 35 & 0 & 0 & 0 & 0 \\
8 & -3 & 0 & 0 & 1 & 36 & -3 & 0 & 0 & -15 \\
9 & -3 & -3 & 0 & -3 & 37 & 6 & 6 & 0 & 6 \\
10 & 0 & 0 & 0 & 0 & 38 & 0 & 0 & 0 & 0 \\
11 & -2 & 0 & -2 & 14 & 39 & 0 & 0 & 0 & 0 \\
12 & 0 & 0 & 0 & 0 & 40 & 0 & 0 & 0 & 0 \\
13 & 0 & 0 & 0 & 0 & 41 & 0 & 0 & 0 & 0 \\
14 & -3 & 0 & 0 & -7 & 42 & 0 & 0 & 0 & 0 \\
15 & 0 & 0 & 0 & 0 & 43 & -2 & 0 & -2 & 14 \\
16 & 1 & 0 & 0 & -11 & 44 & -10 & 0 & 0 & 14 \\
17 & 0 & 0 & 0 & 0 & 45 & 0 & 0 & 0 & 0 \\
18 & -3 & 0 & 0 & 9 & 46 & -6 & 0 & 0 & -14 \\
19 & 0 & 0 & 0 & 0 & 47 & 0 & 0 & 0 & 0 \\
20 & 0 & 0 & 0 & 0 & 48 & 0 & 0 & 0 & 0 \\
21 & 0 & 0 & 0 & 0 & 49 & -7 & -7 & 0 & -7 \\
22 & 6 & 0 & 0 & 14 & 50 & 5 & 0 & 0 & -15 \\
23 & 2 & 0 & 2 & -14 & 51 & 0 & 0 & 0 & 0 \\
24 & 0 & 0 & 0 & 0 & 52 & 0 & 0 & 0 & 0 \\
25 & 5 & 5 & 0 & 5 & 53 & -10 & -10 & 0 & -10 \\
26 & 0 & 0 & 0 & 0 & 54 & 0 & 0 & 0 & 0 \\
27 & 0 & 0 & 0 & 0 & 55 & 0 & 0 & 0 & 0
\end{tabular}




\begin{tabular}{l|lllll|l|llll}
28 & 5 & 0 & 0 & -7 & 56 & 1 & 0 & 0 & 21 \\
\hline
\end{tabular}

Theorem 3.3.1. Let $\left(a_{1}, a_{2}, a_{3}, a_{4}\right)$ be as in the second column of Table 3.1.1. Then $\varphi\left(q^{a_{1}}\right) \varphi\left(q^{a_{2}}\right) \varphi\left(q^{a_{3}}\right) \varphi\left(q^{a_{4}}\right) \in M_{2}\left(\Gamma_{0}(56), \chi_{3}\right)$.

Proof. We appeal to (2.1.9) for each of the six quadratic forms and then check the conditions (L1), (L2) and (L3) of Theorem 3.1.1 for each quadratic form. We have $N=56$. First we consider $(1,1,1,7)$

$$
\varphi^{3}(q) \varphi\left(q^{7}\right)=\frac{\eta^{15}(2 z) \eta^{5}(14 z)}{\eta^{6}(z) \eta^{6}(4 z) \eta^{2}(7 z) \eta^{2}(28 z)}
$$

Then we have

Table 3.3.2(a)

\begin{tabular}{r|rrrrrr}
\hline$\delta$ & 1 & 2 & 4 & 7 & 14 & 28 \\
\hline$r_{\delta}$ & -6 & 15 & -6 & -2 & 5 & -2 \\
\hline
\end{tabular}

It can be seen from Table 3.3.2(a) that conditions $(L 1)$ and $(L 2)$ are satisfied.

Table 3.3.2(b)

\begin{tabular}{r|rrrrrrrr}
\hline$d \mid 56$ & 1 & 2 & 4 & 7 & 8 & 14 & 28 & 56 \\
\hline$\sum_{1 \leq \delta \mid 56} \frac{\operatorname{gcd}(d, \delta)^{2} \cdot r_{\delta}}{\delta}$ & 0 & $132 / 7$ & 0 & 0 & 0 & 60 & 0 & 0 \\
\hline
\end{tabular}

From Table 3.3.2(b) the condition (L3) is also satisfied. Thus $\varphi^{3}(q) \varphi\left(q^{7}\right)$ $\in M_{2}\left(\Gamma_{0}(56), \chi_{3}\right)$. Now for the form $(1,2,2,7)$ we have

$$
\varphi(q) \varphi^{2}\left(q^{2}\right) \varphi\left(q^{7}\right)=\frac{\eta(2 z) \eta^{8}(4 z) \eta^{5}(14 z)}{\eta^{2}(z) \eta^{2}(7 z) \eta^{4}(8 z) \eta^{2}(28 z)}
$$

Then we have 
Table 3.3.3(a)

\begin{tabular}{r|rrrrrrr}
\hline$\delta$ & 1 & 2 & 4 & 7 & 8 & 14 & 28 \\
\hline$r_{\delta}$ & -2 & 1 & 8 & -2 & -4 & 5 & -2 \\
\hline
\end{tabular}

It can be seen from Table 3.3.3(a) that conditions $(L 1)$ and $(L 2)$ are satisfied.

Table 3.3.3(b)

\begin{tabular}{r|rrrrrrrr}
\hline$d \mid 56$ & 1 & 2 & 4 & 7 & 8 & 14 & 28 & 56 \\
\hline$\sum_{1 \leq \delta \mid 56} \frac{\operatorname{gcd}(d, \delta)^{2} \cdot r_{\delta}}{\delta}$ & 0 & $48 / 7$ & 24 & 0 & 0 & 48 & 24 & 0 \\
\hline
\end{tabular}

From Table 3.3.3(b) the condition (L3) is also satisfied. Thus $\varphi(q) \varphi^{2}\left(q^{2}\right) \varphi\left(q^{7}\right) \in$ $M_{2}\left(\Gamma_{0}(56), \chi_{3}\right)$. Then for the form $(1,7,7,7)$ we have

$$
\varphi(q) \varphi^{3}\left(q^{7}\right)=\frac{\eta^{5}(2 z) \eta^{15}(14 z)}{\eta^{2}(z) \eta^{2}(4 z) \eta^{6}(7 z) \eta^{6}(28 z)}
$$

Then we have

Table 3.3.4(a)

\begin{tabular}{r|rrrrrr}
\hline$\delta$ & 1 & 2 & 4 & 7 & 14 & 28 \\
\hline$r_{\delta}$ & -2 & 5 & -2 & -6 & 15 & -6 \\
\hline
\end{tabular}

It can be seen from Table 3.3.4(a) that conditions $(L 1)$ and $(L 2)$ are satisfied.

Table 3.3.4(b)

\begin{tabular}{r|rrrrrrrr}
\hline$d \mid 56$ & 1 & 2 & 4 & 7 & 8 & 14 & 28 & 56 \\
\hline$\sum_{1 \leq \delta \mid 56} \frac{\operatorname{gcd}(d, \delta)^{2} \cdot r_{\delta}}{\delta}$ & 0 & $60 / 7$ & 0 & 0 & 0 & 132 & 0 & 0 \\
\hline
\end{tabular}

From Table 3.3.4(b) the condition (L3) is also satisfied. Thus $\varphi(q) \varphi^{3}\left(q^{7}\right) \in$ $M_{2}\left(\Gamma_{0}(56), \chi_{3}\right)$. Now for the form $(1,1,2,14)$ we have

$$
\varphi^{2}(q) \varphi\left(q^{2}\right) \varphi\left(q^{14}\right)=\frac{\eta^{8}(2 z) \eta(4 z) \eta^{5}(28 z)}{\eta^{4}(z) \eta^{2}(8 z) \eta^{2}(14 z) \eta^{2}(56 z)}
$$

Then we have 
Table 3.3.5(a)

\begin{tabular}{r|rrrrrrr}
\hline$\delta$ & 1 & 2 & 4 & 8 & 14 & 28 & 56 \\
\hline$r_{\delta}$ & -4 & 8 & 1 & -2 & -2 & 5 & -2 \\
\hline
\end{tabular}

It can be seen from Table 3.3.5(a) that conditions $(L 1)$ and $(L 2)$ are satisfied.

Table 3.3.5(b)

\begin{tabular}{r|rrrrrrrr}
\hline$d \mid 56$ & 1 & 2 & 4 & 7 & 8 & 14 & 28 & 56 \\
\hline$\sum_{1 \leq \delta \mid 56} \frac{\operatorname{gcd}(d, \delta)^{2} \cdot r_{\delta}}{\delta}$ & 0 & 12 & $96 / 7$ & 0 & 0 & 12 & 96 & 0 \\
\hline
\end{tabular}

From Table 3.3.5(b) the condition (L3) is also satisfied. Thus $\varphi^{2}(q) \varphi\left(q^{2}\right) \varphi\left(q^{14}\right) \in$ $M_{2}\left(\Gamma_{0}(56), \chi_{3}\right)$. Now for the form $(2,7,7,14)$ we have

$$
\varphi\left(q^{2}\right) \varphi^{2}\left(q^{7}\right) \varphi\left(q^{14}\right)=\frac{\eta^{5}(4 z) \eta^{8}(14 z) \eta(28 z)}{\eta^{2}(2 z) \eta^{4}(7 z) \eta^{2}(8 z) \eta^{2}(56 z)}
$$

Then we have

Table 3.3.6(a)

\begin{tabular}{r|rrrrrrr}
\hline$\delta$ & 2 & 4 & 7 & 8 & 14 & 28 & 56 \\
\hline$r_{\delta}$ & -2 & 5 & -4 & -2 & 8 & 1 & -2 \\
\hline
\end{tabular}

It can be seen from Table 3.3.6(a) that conditions $(L 1)$ and $(L 2)$ are satisfied.

Table 3.3.6(b)

\begin{tabular}{r|rrrrrrrr}
\hline$d \mid 56$ & 1 & 2 & 4 & 7 & 8 & 14 & 28 & 56 \\
\hline$\sum_{1 \leq \delta \mid 56} \frac{\operatorname{gcd}(d, \delta)^{2} \cdot r_{\delta}}{\delta}$ & 0 & $12 / 7$ & $96 / 7$ & 0 & 0 & 84 & 96 & 0 \\
\hline
\end{tabular}

From Table 3.3.6(b) the condition (L3) is also satisfied. Thus $\varphi\left(q^{2}\right) \varphi^{2}\left(q^{7}\right) \varphi\left(q^{14}\right) \in$ $M_{2}\left(\Gamma_{0}(56), \chi_{3}\right)$. Now for the form $(1,7,14,14)$ we have

$$
\varphi(q) \varphi\left(q^{7}\right) \varphi^{2}\left(q^{14}\right)=\frac{\eta^{5}(2 z) \eta(14 z) \eta^{8}(28 z)}{\eta^{2}(z) \eta^{2}(4 z) \eta^{2}(7 z) \eta^{4}(56 z)}
$$

Then we have 
Table 3.3.7(a)

\begin{tabular}{r|rrrrrrr}
\hline$\delta$ & 1 & 2 & 4 & 7 & 14 & 28 & 56 \\
\hline$r_{\delta}$ & -2 & 5 & -2 & -2 & 1 & 8 & -4 \\
\hline
\end{tabular}

It can be seen from Table 3.3.7(a) that conditions $(L 1)$ and $(L 2)$ are satisfied.

Table 3.3.7(b)

\begin{tabular}{r|rrrrrrrr}
\hline$d \mid 56$ & 1 & 2 & 4 & 7 & 8 & 14 & 28 & 56 \\
\hline$\sum_{1 \leq \delta \mid 56} \frac{\operatorname{gcd}(d, \delta)^{2} \cdot r_{\delta}}{\delta}$ & 0 & $48 / 7$ & $24 / 7$ & 0 & 0 & 48 & 168 & 0 \\
\hline
\end{tabular}

From Table 3.3.7(b) the condition (L3) is also satisfied. Thus $\varphi(q) \varphi\left(q^{7}\right) \varphi^{2}\left(q^{14}\right) \in$ $M_{2}\left(\Gamma_{0}(56), \chi_{3}\right)$.

Theorem 3.3.2. $B_{k}(q)(1 \leq k \leq 4)$ given by $(3.3 .5)-(3.3 .8)$ are in $S_{2}\left(\Gamma_{0}(56), \chi_{3}\right)$.

Proof. We will check conditions (L1),(L2) and (L4) of Theorem 3.1.1. We have $N=56$. First we consider

$$
B_{1}(q)=\frac{\eta^{2}(2 z) \eta^{3}(7 z)}{\eta(z)}
$$

Then we have

\begin{tabular}{r|rrr}
\multicolumn{4}{c}{ Table 3.3.8(a) } \\
\hline$\delta$ & 1 & 2 & 7 \\
\hline$r_{\delta}$ & -1 & 2 & 3 \\
\hline
\end{tabular}

It can be seen from Table 3.3.8(a) that conditions (L1) and (L2) are satisfied.

Table 3.3.8(b)

\begin{tabular}{r|rrrrrrrr}
\hline$d \mid 56$ & 1 & 2 & 4 & 7 & 8 & 14 & 28 & 56 \\
\hline$\sum_{1 \leq \delta \mid 56} \frac{\operatorname{gcd}(d, \delta)^{2} \cdot r_{\delta}}{\delta}$ & $3 / 7$ & $24 / 7$ & $24 / 7$ & 21 & $24 / 7$ & 24 & 24 & 24 \\
\hline
\end{tabular}


From Table 3.3.8(b) the condition $(L 4)$ is also satisfied. Thus $B_{1}(q) \in S_{2}\left(\Gamma_{0}(56), \chi_{3}\right)$. Secondly we consider

$$
B_{2}(q)=\frac{\eta^{3}(8 z) \eta^{2}(28 z)}{\eta(56 z)}
$$

Then we have

\begin{tabular}{|c|c|c|c|}
\hline \multicolumn{4}{|c|}{ Table 3.3.9(a) } \\
\hline$\delta$ & 8 & 28 & \\
\hline$r_{\delta}$ & 3 & 2 & - \\
\hline
\end{tabular}

It can be seen from Table 3.3.9(a) that conditions (L1) and (L2) are satisfied.

Table 3.3.9(b)

\begin{tabular}{r|rrrrrrrr}
\hline$d \mid 56$ & 1 & 2 & 4 & 7 & 8 & 14 & 28 & 56 \\
\hline$\sum_{1 \leq \delta \mid 56} \frac{\operatorname{gcd}(d, \delta)^{2} \cdot r_{\delta}}{\delta}$ & $3 / 7$ & $12 / 7$ & $48 / 7$ & 3 & 24 & 12 & 48 & 24 \\
\hline
\end{tabular}

From Table 3.3.9(b) the condition $(L 4)$ is also satisfied. Thus $B_{2}(q) \in S_{2}\left(\Gamma_{0}(56), \chi_{3}\right)$.

Thirdly we consider

$$
B_{3}(q)=\frac{\eta^{2}(4 z) \eta^{3}(56 z)}{\eta(8 z)}
$$

\begin{tabular}{|c|c|c|c|}
\hline \multicolumn{4}{|c|}{ Table 3.3.10(a) } \\
\hline$\delta$ & 4 & & 5 \\
\hline$r_{\delta}$ & 2 & -1 & : \\
\hline
\end{tabular}

Then we have

It can be seen from Table 3.3.10(a) that conditions (L1) and (L2) are satisfied.

$$
\text { Table 3.3.10(b) }
$$

\begin{tabular}{r|rrrrrrrr}
\hline$d \mid 56$ & 1 & 2 & 4 & 7 & 8 & 14 & 28 & 56 \\
\hline$\sum_{1 \leq \delta \mid 56} \frac{\operatorname{gcd}(d, \delta)^{2} \cdot r_{\delta}}{\delta}$ & $3 / 7$ & $12 / 7$ & $48 / 7$ & 3 & $24 / 7$ & 12 & 48 & 168 \\
\hline
\end{tabular}


From Table 3.3.10(b) the condition $(L 4)$ is also satisfied. Thus $B_{3}(q) \in S_{2}\left(\Gamma_{0}(56), \chi_{3}\right)$. Fourthly we consider

$$
B_{4}(q)=\frac{\eta^{3}(z) \eta^{2}(14 z)}{\eta(7 z)}
$$

Then we have

Table 3.3.11(a)

\begin{tabular}{r|rrr}
\hline$\delta$ & 1 & 7 & 14 \\
\hline$r_{\delta}$ & 3 & -1 & 2 \\
\hline
\end{tabular}

It can be seen from Table 3.3.11(a) that conditions (L1) and (L2) are satisfied.

Table 3.3.11(b)

\begin{tabular}{r|rrrrrrrr}
\hline$d \mid 56$ & 1 & 2 & 4 & 7 & 8 & 14 & 28 & 56 \\
\hline$\sum_{1 \leq \delta \mid 56} \frac{\operatorname{gcd}(d, \delta)^{2} \cdot r_{\delta}}{\delta}$ & 3 & $24 / 7$ & $24 / 7$ & 3 & $24 / 7$ & 24 & 24 & 24 \\
\hline
\end{tabular}

From Table 3.3.11(b) the condition $(L 4)$ is also satisfied. Thus $B_{4}(q) \in$ $S_{2}\left(\Gamma_{0}(56), \chi_{3}\right)$

Theorem 3.3.3. (a) $\left\{B_{1}(q), B_{2}(q), B_{3}(q), B_{4}(q)\right\}$ is a basis for $S_{2}\left(\Gamma_{0}(56), \chi_{3}\right)$.

(b) $\left\{E_{2, \chi_{3}, \chi_{0}}\left(q^{t}\right), E_{2, \chi_{0}, \chi_{3}}\left(q^{t}\right), E_{2, \chi_{1}, \chi_{2}}\left(q^{t}\right), E_{2, \chi_{2}, \chi_{1}}\left(q^{t}\right) \mid t=1,2\right\}$ is a basis for $E_{2}\left(\Gamma_{0}(56), \chi_{3}\right)$.

(c) $\left\{E_{2, \chi_{3}, \chi_{0}}\left(q^{t}\right), E_{2, \chi_{0}, \chi_{3}}\left(q^{t}\right), E_{2, \chi_{1}, \chi_{2}}\left(q^{t}\right), E_{2, \chi_{2}, \chi_{1}}\left(q^{t}\right) \mid t=1,2\right\}$ together with $B_{k}(q)$ $(1 \leq k \leq 4)$ constitute a basis for $M_{2}\left(\Gamma_{0}(56), \chi_{3}\right)$.

Proof. (a) By Theorem 3.3.2, $B_{k}(q)(1 \leq k \leq 4) \in S_{2}\left(\Gamma_{0}(56), \chi_{3}\right)$. There is no linear relationship among them. By Example 2.3.3, we have $\operatorname{dim} S_{2}\left(\Gamma_{0}(56), \chi_{3}\right)=4$. Therefore, $B_{k}(q)(1 \leq k \leq 4)$ constitute a basis for $S_{2}\left(\Gamma_{0}(56), \chi_{3}\right)$.

(b) By Example 2.3.3, we have $\operatorname{dim} E_{2}\left(\Gamma_{0}(56), \chi_{3}\right)=8$. By Theorem 2.2.3, $\left\{E_{2, \chi_{3}, \chi_{0}}\left(q^{t}\right), E_{2, \chi_{0}, \chi_{3}}\left(q^{t}\right), E_{2, \chi_{1}, \chi_{2}}\left(q^{t}\right), E_{2, \chi_{2}, \chi_{1}}\left(q^{t}\right) \mid t=1,2\right\}$ is a basis for $E_{2}\left(\Gamma_{0}(56), \chi_{3}\right)$.

(c) By Example 2.3.3, we have $\operatorname{dim} M_{2}\left(\Gamma_{0}(56), \chi_{3}\right)=12$. Therefore, by $(2.1 .1)$ 
$\left\{E_{2, \chi_{3}, \chi_{0}}\left(q^{t}\right), E_{2, \chi_{0}, \chi_{3}}\left(q^{t}\right), E_{2, \chi_{1}, \chi_{2}}\left(q^{t}\right), E_{2, \chi_{2}, \chi_{1}}\left(q^{t}\right) \mid t=1,2\right\}$ together with $B_{k}(q)(1 \leq$ $k \leq 4)$ constitute a basis for $M_{2}\left(\Gamma_{0}(56), \chi_{3}\right)$.

Theorem 3.3.4. Let $\chi_{0}$ be the trivial character and $\chi_{1}, \chi_{2}, \chi_{3}$ be as in (3.1.3). Then

$$
\begin{aligned}
& \text { (a) } \varphi^{3}(q) \varphi\left(q^{7}\right)=\frac{7}{2} E_{2, \chi_{3}, \chi_{0}}(q)-\frac{1}{4} E_{2, \chi_{0}, \chi_{3}}(q)+\frac{7}{4} E_{2, \chi_{1}, \chi_{2}}(q)-\frac{1}{2} E_{2, \chi_{2}, \chi_{1}}(q) \\
& +3 B_{2}(q)-21 B_{3}(q)-\frac{3}{2} B_{4}(q) \\
& \text { (b) } \varphi(q) \varphi^{2}\left(q^{2}\right) \varphi\left(q^{7}\right)=\frac{7}{4} E_{2, \chi_{3}, \chi_{0}}(q)-\frac{1}{4} E_{2, \chi_{0}, \chi_{3}}\left(q^{2}\right)+\frac{7}{4} E_{2, \chi_{1}, \chi_{2}}\left(q^{2}\right)-\frac{1}{4} E_{2, \chi_{2}, \chi_{1}}(q) \\
& -\frac{7}{8} B_{1}(q)+\frac{3}{2} B_{2}(q)-\frac{21}{2} B_{3}(q)-\frac{1}{8} B_{4}(q) \\
& \text { (c) } \varphi(q) \varphi^{3}\left(q^{7}\right)=\frac{1}{2} E_{2, \chi_{3}, \chi_{0}}(q)-\frac{1}{4} E_{2, \chi_{0}, \chi_{3}}(q)-\frac{1}{4} E_{2, \chi_{1}, \chi_{2}}(q)+\frac{1}{2} E_{2, \chi_{2}, \chi_{1}}(q) \\
& -\frac{3}{2} B_{1}(q)+3 B_{2}(q)+3 B_{3}(q) \\
& \text { (d) } \varphi^{2}(q) \varphi\left(q^{2}\right) \varphi\left(q^{14}\right)=\frac{7}{4} E_{2, \chi_{3}, \chi_{0}}(q)-\frac{1}{4} E_{2, \chi_{0}, \chi_{3}}\left(q^{2}\right)+\frac{7}{4} E_{2, \chi_{1}, \chi_{2}}\left(q^{2}\right)-\frac{1}{4} E_{2, \chi_{2}, \chi_{1}}(q) \\
& +\frac{21}{8} B_{1}(q)-\frac{1}{2} B_{2}(q)+\frac{7}{2} B_{3}(q)+\frac{3}{8} B_{4}(q), \\
& \text { (e) } \varphi\left(q^{2}\right) \varphi^{2}\left(q^{7}\right) \varphi\left(q^{14}\right)=\frac{1}{4} E_{2, \chi_{3}, \chi_{0}}(q)-\frac{1}{4} E_{2, \chi_{0}, \chi_{3}}\left(q^{2}\right)-\frac{1}{4} E_{2, \chi_{1}, \chi_{2}}\left(q^{2}\right)+\frac{1}{4} E_{2, \chi_{2}, \chi_{1}}(q) \\
& +\frac{3}{8} B_{1}(q)-\frac{1}{2} B_{2}(q)-\frac{1}{2} B_{3}(q)-\frac{3}{8} B_{4}(q), \\
& \text { (f) } \varphi(q) \varphi\left(q^{7}\right) \varphi^{2}\left(q^{14}\right)=\frac{1}{4} E_{2, \chi_{3}, \chi_{0}}(q)-\frac{1}{4} E_{2, \chi_{0}, \chi_{3}}\left(q^{2}\right)-\frac{1}{4} E_{2, \chi_{1}, \chi_{2}}\left(q^{2}\right)+\frac{1}{4} E_{2, \chi_{2}, \chi_{1}}(q) \\
& -\frac{1}{8} B_{1}(q)+\frac{3}{2} B_{2}(q)+\frac{3}{2} B_{3}(q)+\frac{1}{8} B_{4}(q) \text {. }
\end{aligned}
$$

Proof. Let $\left(a_{1}, a_{2}, a_{3}, a_{4}\right)$ be one of the quadratic forms listed in the second column of Table 3.1.1. By Theorem 3.3.1 we have $\varphi\left(q^{a_{1}}\right) \varphi\left(q^{a_{2}}\right) \varphi\left(q^{a_{3}}\right) \varphi\left(q^{a_{4}}\right) \in M_{2}\left(\Gamma_{0}(56), \chi_{3}\right)$. Therefore, by Theorem 3.3.3 (c), $\varphi\left(q^{a_{1}}\right) \varphi\left(q^{a_{2}}\right) \varphi\left(q^{a_{3}}\right) \varphi\left(q^{a_{4}}\right)$ must be a linear combination of $\left\{E_{2, \chi_{3}, \chi_{0}}\left(q^{t}\right), E_{2, \chi_{0}, \chi_{3}}\left(q^{t}\right), E_{2, \chi_{1}, \chi_{2}}\left(q^{t}\right), E_{2, \chi_{2}, \chi_{1}}\left(q^{t}\right) \mid t=1,2\right\}$ and $B_{k}(q)(1 \leq$ 
$k \leq 4)$, namely

$$
\begin{aligned}
\varphi\left(q^{a_{1}}\right) \varphi\left(q^{a_{2}}\right) \varphi\left(q^{a_{3}}\right) \varphi\left(q^{a_{4}}\right)= & x_{1} E_{2, \chi_{3}, \chi_{0}}(q)+x_{2} E_{2, \chi_{3}, \chi_{0}}\left(q^{2}\right)+x_{3} E_{2, \chi_{0}, \chi_{3}}(q) \\
& +x_{4} E_{2, \chi_{0}, \chi_{3}}\left(q^{2}\right)+x_{5} E_{2, \chi_{1}, \chi_{2}}(q)+x_{6} E_{2, \chi_{1}, \chi_{2}}\left(q^{2}\right) \\
& +x_{7} E_{2, \chi_{2}, \chi_{1}}(q)+x_{8} E_{2, \chi_{2}, \chi_{1}}\left(q^{2}\right)+y_{1} B_{1}(q)+y_{2} B_{2}(q) \\
& +y_{3} B_{3}(q)+y_{4} B_{4}(q) .
\end{aligned}
$$

We equate the first twenty coefficients of $q^{n}$ on both sides of the equation above to obtain a system of linear equations with the unknowns $x_{1}, x_{2}, x_{3}, x_{4}, x_{5}, x_{6}, x_{7}, x_{8}$ and $y_{1}, y_{2}, y_{3}, y_{4}$. Then, using MAPLE we solve the system to find the asserted coefficients.

Theorem 3.3.5. Let $n \in \mathbb{N}$. Let $\sigma_{\chi_{i}, \chi_{j}}(n)$ be as in (2.2.1) for $i, j \in\{0,1,2,3\}$. Then

(a) $N(1,1,1,7 ; n)=\frac{7}{2} \sigma_{\chi_{3}, \chi_{0}}(n)-\frac{1}{4} \sigma_{\chi_{0}, \chi_{3}}(n)+\frac{7}{4} \sigma_{\chi_{1}, \chi_{2}}(n)-\frac{1}{2} \sigma_{\chi_{2}, \chi_{1}}(n)+3 b_{2}(n)$

$$
-21 b_{3}(n)-\frac{3}{2} b_{4}(n)
$$

(b) $N(1,2,2,7 ; n)=\frac{7}{4} \sigma_{\chi_{3}, \chi_{0}}(n)-\frac{1}{4} \sigma_{\chi_{0}, \chi_{3}}(n / 2)+\frac{7}{4} \sigma_{\chi_{1}, \chi_{2}}(n / 2)-\frac{1}{4} \sigma_{\chi_{2}, \chi_{1}}(n)$ $-\frac{7}{8} b_{1}(n)+\frac{3}{2} b_{2}(n)-\frac{21}{2} b_{3}(n)-\frac{1}{8} b_{4}(n)$,

(c) $N(1,7,7,7 ; n)=\frac{1}{2} \sigma_{\chi_{3}, \chi_{0}}(n)-\frac{1}{4} \sigma_{\chi_{0}, \chi_{3}}(n)-\frac{1}{4} \sigma_{\chi_{1}, \chi_{2}}(n)+\frac{1}{2} \sigma_{\chi_{2}, \chi_{1}}(n)-\frac{3}{2} b_{1}(n)$

$$
+3 b_{2}(n)+3 b_{3}(n)
$$

(d) $N(1,1,2,14 ; n)=\frac{7}{4} \sigma_{\chi_{3}, \chi_{0}}(n)-\frac{1}{4} \sigma_{\chi_{0}, \chi_{3}}(n / 2)+\frac{7}{4} \sigma_{\chi_{1}, \chi_{2}}(n / 2)-\frac{1}{4} \sigma_{\chi_{2}, \chi_{1}}(n)$

$$
+\frac{21}{8} b_{1}(n)-\frac{1}{2} b_{2}(n)+\frac{7}{2} b_{3}(n)+\frac{3}{8} b_{4}(n),
$$

(e) $N(2,7,7,14 ; n)=\frac{1}{4} \sigma_{\chi_{3}, \chi_{0}}(n)-\frac{1}{4} \sigma_{\chi_{0}, \chi_{3}}(n / 2)-\frac{1}{4} \sigma_{\chi_{1}, \chi_{2}}(n / 2)+\frac{1}{4} \sigma_{\chi_{2}, \chi_{1}}(n)$

$$
+\frac{3}{8} b_{1}(n)-\frac{1}{2} b_{2}(n)-\frac{1}{2} b_{3}(n)-\frac{3}{8} b_{4}(n)
$$


(f) $N(1,7,14,14 ; n)=\frac{1}{4} \sigma_{\chi_{3}, \chi_{0}}(n)-\frac{1}{4} \sigma_{\chi_{0}, \chi_{3}}(n / 2)-\frac{1}{4} \sigma_{\chi_{1}, \chi_{2}}(n / 2)+\frac{1}{4} \sigma_{\chi_{2}, \chi_{1}}(n)$

$$
-\frac{1}{8} b_{1}(n)+\frac{3}{2} b_{2}(n)+\frac{3}{2} b_{3}(n)+\frac{1}{8} b_{4}(n)
$$

Proof. From (2.1.6), (3.3.1)-(3.3.4) and Theorem 3.3.4, we obtain

(a) $\sum_{n=0}^{\infty} N(1,1,1,7 ; n) q^{n}=\varphi^{3}(q) \varphi\left(q^{7}\right)$

$=\frac{7}{2} E_{2, \chi_{3}, \chi_{0}}(q)-\frac{1}{4} E_{2, \chi_{0}, \chi_{3}}(q)+\frac{7}{4} E_{2, \chi_{1}, \chi_{2}}(q)-\frac{1}{2} E_{2, \chi_{2}, \chi_{1}}(q)+3 B_{2}(q)-21 B_{3}(q)$ $-\frac{3}{2} B_{4}(q)$

$=1+\sum_{n=1}^{\infty}\left(\frac{7}{2} \sigma_{\chi_{3}, \chi_{0}}(n)-\frac{1}{4} \sigma_{\chi_{0}, \chi_{3}}(n)+\frac{7}{4} \sigma_{\chi_{1}, \chi_{2}}(n)-\frac{1}{2} \sigma_{\chi_{2}, \chi_{1}}(n)+3 b_{2}(n)-21 b_{3}(n)\right.$ $\left.-\frac{3}{2} b_{4}(n)\right) q^{n}$

(b) $\sum_{n=0}^{\infty} N(1,2,2,7 ; n) q^{n}=\varphi(q) \varphi^{2}\left(q^{2}\right) \varphi\left(q^{7}\right)$

$=\frac{7}{4} E_{2, \chi_{3}, \chi_{0}}(q)-\frac{1}{4} E_{2, \chi_{0}, \chi_{3}}\left(q^{2}\right)+\frac{7}{4} E_{2, \chi_{1}, \chi_{2}}\left(q^{2}\right)-\frac{1}{4} E_{2, \chi_{2}, \chi_{1}}(q)-\frac{7}{8} B_{1}(q)+\frac{3}{2} B_{2}(q)$ $-\frac{21}{2} B_{3}(q)-\frac{1}{8} B_{4}(q)$

$=1+\sum_{n=1}^{\infty}\left(\frac{7}{4} \sigma_{\chi_{3}, \chi_{0}}(n)-\frac{1}{4} \sigma_{\chi_{0}, \chi_{3}}(n / 2)+\frac{7}{4} \sigma_{\chi_{1}, \chi_{2}}(n / 2)-\frac{1}{4} \sigma_{\chi_{2}, \chi_{1}}(n)-\frac{7}{8} b_{1}(n)\right.$ $\left.+\frac{3}{2} b_{2}(n)-\frac{21}{2} b_{3}(n)-\frac{1}{8} b_{4}(n)\right) q^{n}$

(c) $\sum_{n=0}^{\infty} N(1,7,7,7 ; n) q^{n}=\varphi(q) \varphi^{3}\left(q^{7}\right)$

$=\frac{1}{2} E_{2, \chi_{3}, \chi_{0}}(q)-\frac{1}{4} E_{2, \chi_{0}, \chi_{3}}(q)-\frac{1}{4} E_{2, \chi_{1}, \chi_{2}}(q)+\frac{1}{2} E_{2, \chi_{2}, \chi_{1}}(q)-\frac{3}{2} B_{1}(q)+3 B_{2}(q)$ $+3 B_{3}(q)$

$=1+\sum_{n=1}^{\infty}\left(-\frac{1}{2} \sigma_{\chi_{3}, \chi_{0}}(n)-\frac{1}{4} \sigma_{\chi_{0}, \chi_{3}}(n)-\frac{1}{4} \sigma_{\chi_{1}, \chi_{2}}(n)+\frac{1}{2} \sigma_{\chi_{2}, \chi_{1}}(n)-\frac{3}{2} b_{1}(n)\right.$

$\left.+3 b_{2}(n)+3 b_{3}(n)\right) q^{n}$ 


$$
\begin{aligned}
& \text { (d) } \sum_{n=0}^{\infty} N(1,1,2,14 ; n) q^{n}=\varphi^{2}(q) \varphi\left(q^{2}\right) \varphi\left(q^{14}\right) \\
& =\frac{7}{4} E_{2, \chi_{3}, \chi_{0}}(q)-\frac{1}{4} E_{2, \chi_{0}, \chi_{3}}\left(q^{2}\right)+\frac{7}{4} E_{2, \chi_{1}, \chi_{2}}\left(q^{2}\right)-\frac{1}{4} E_{2, \chi_{2}, \chi_{1}}(q)+\frac{21}{8} B_{1}(q) \\
& -\frac{1}{2} B_{2}(q)+\frac{7}{2} B_{3}(q)+\frac{3}{8} B_{4}(q) \\
& =1+\sum_{n=1}^{\infty}\left(\frac{7}{4} \sigma_{\chi_{3}, \chi_{0}}(n)-\frac{1}{4} \sigma_{\chi_{0}, \chi_{3}}(n / 2)+\frac{7}{4} \sigma_{\chi_{1}, \chi_{2}}(n / 2)-\frac{1}{4} \sigma_{\chi_{2}, \chi_{1}}(n)+\frac{21}{8} b_{1}(n)\right. \\
& \left.-\frac{1}{2} b_{2}(n)+\frac{7}{2} b_{3}(n)+\frac{3}{8} b_{4}(n)\right) q^{n} \\
& \text { (e) } \sum_{n=0}^{\infty} N(2,7,7,14 ; n) q^{n}=\varphi(q) \varphi^{2}\left(q^{7}\right) \varphi\left(q^{14}\right) \\
& =\frac{1}{4} E_{2, \chi_{3}, \chi_{0}}(q)-\frac{1}{4} E_{2, \chi_{0}, \chi_{3}}\left(q^{2}\right)-\frac{1}{4} E_{2, \chi_{1}, \chi_{2}}\left(q^{2}\right)+\frac{1}{4} E_{2, \chi_{2}, \chi_{1}}(q)+\frac{3}{8} B_{1}(q)-\frac{1}{2} B_{2}(q) \\
& -\frac{1}{2} B_{3}(q)-\frac{3}{8} B_{4}(q)-\frac{1}{2} D_{1}(q)-\frac{7}{5} D_{3}(q)-\frac{12}{5} D_{4}(q)+\frac{1}{2} D_{5}(q)+\frac{7}{5} D_{6}(q), \\
& =1+\sum_{n=1}^{\infty}\left(\frac{1}{4} \sigma_{\chi_{3}, \chi_{0}}(n)-\frac{1}{4} \sigma_{\chi_{0}, \chi_{3}}(n / 2)-\frac{1}{4} \sigma_{\chi_{1}, \chi_{2}}(n / 2)+\frac{1}{4} \sigma_{\chi_{2}, \chi_{1}}(n)+\frac{3}{8} b_{1}(n)\right. \\
& \left.-\frac{1}{2} b_{2}(n)-\frac{1}{2} b_{3}(n)-\frac{3}{8} b_{4}(n)\right) q^{n} \\
& \text { (f) } \sum_{n=0}^{\infty} N(1,7,14,14 ; n) q^{n}=\varphi(q) \varphi\left(q^{7}\right) \varphi^{2}\left(q^{14}\right) \\
& =\frac{1}{4} E_{2, \chi_{3}, \chi_{0}}(q)-\frac{1}{4} E_{2, \chi_{0}, \chi_{3}}\left(q^{2}\right)-\frac{1}{4} E_{2, \chi_{1}, \chi_{2}}\left(q^{2}\right)+\frac{1}{4} E_{2, \chi_{2}, \chi_{1}}(q)-\frac{1}{8} B_{1}(q)+\frac{3}{2} B_{2}(q) \\
& +\frac{3}{2} B_{3}(q)+\frac{1}{8} B_{4}(q), \\
& =1+\sum_{n=1}^{\infty}\left(\frac{1}{4} \sigma_{\chi_{3}, \chi_{0}}(n)-\frac{1}{4} \sigma_{\chi_{0}, \chi_{3}}(n / 2)-\frac{1}{4} \sigma_{\chi_{1}, \chi_{2}}(n / 2)+\frac{1}{4} \sigma_{\chi_{2}, \chi_{1}}(n)-\frac{1}{8} b_{1}(n)\right. \\
& \left.+\frac{3}{2} b_{2}(n)+\frac{3}{2} b_{3}(n)+\frac{1}{8} b_{4}(n)\right) q^{n} \text {. }
\end{aligned}
$$

Equating the coefficients of $q^{n}$ on both sides of equations (a)-(f) yields the results.

The values of $N\left(a_{1}, a_{2}, a_{3}, a_{4} ; n\right)$ for $1 \leq n \leq 20$ for the quadratic forms $\left(a_{1}, a_{2}, a_{3}, a_{4}\right)$ in Theorem 3.3.5 are given in Table 3.3.12. One can verify them by using Table 3.3.1. 
Table 3.3 .12

\begin{tabular}{l|rrrrrrrrrrrrrrrrrrrr}
\hline \multicolumn{1}{c|}{$n$} & 1 & 2 & 3 & 4 & 5 & 6 & 7 & 8 & 9 & 10 & 11 & 12 & 13 & 14 & 15 & 16 & 17 & 18 & 19 & 20 \\
\hline$N(1,1,1,7 ; n)$ & 6 & 12 & 8 & 6 & 24 & 24 & 2 & 24 & 54 & 40 & 36 & 56 & 72 & 48 & 24 & 66 & 96 & 84 & 40 & 72 \\
$N(1,2,2,7 ; n)$ & 2 & 4 & 8 & 6 & 8 & 8 & 2 & 16 & 18 & 24 & 36 & 24 & 24 & 16 & 24 & 26 & 32 & 60 & 40 & 40 \\
$N(1,7,7,7 ; n)$ & 2 & 0 & 0 & 2 & 0 & 0 & 6 & 12 & 2 & 0 & 12 & 0 & 0 & 12 & 24 & 14 & 0 & 24 & 0 & 0 \\
$N(1,1,2,14 ; n)$ & 4 & 6 & 8 & 12 & 8 & 8 & 16 & 6 & 12 & 24 & 8 & 24 & 24 & 2 & 24 & 24 & 32 & 54 & 40 & 40 \\
$N(2,7,7,14 ; n)$ & 0 & 2 & 0 & 0 & 0 & 0 & 4 & 2 & 8 & 0 & 0 & 0 & 0 & 6 & 8 & 12 & 0 & 2 & 0 & 0 \\
$N(1,7,14,14 ; n)$ & 2 & 0 & 0 & 2 & 0 & 0 & 2 & 4 & 2 & 0 & 4 & 0 & 0 & 4 & 8 & 6 & 0 & 8 & 0 & 0 \\
\hline
\end{tabular}

For example, by substituting $n=16$ in Theorem 3.3.5(b), we obtain

$$
\begin{aligned}
N(1,2,2,7 ; 16)= & \frac{7}{4} \sigma_{\chi_{3}, \chi_{0}}(16)-\frac{1}{4} \sigma_{\chi_{0}, \chi_{3}}(8)+\frac{7}{4} \sigma_{\chi_{1}, \chi_{2}}(8)-\frac{1}{4} \sigma_{\chi_{2}, \chi_{1}}(16) \\
& -\frac{7}{8} b_{1}(16)+\frac{3}{2} b_{2}(16)-\frac{21}{2} b_{3}(16)-\frac{1}{8} b_{4}(16)
\end{aligned}
$$

Then appealing to (2.2.1) and (3.1.3), we obtain

$$
\sigma_{\chi_{3}, \chi_{0}}(16)=16, \quad \sigma_{\chi_{0}, \chi_{3}}(8)=1, \quad \sigma_{\chi_{1}, \chi_{2}}(8)=1, \quad \sigma_{\chi_{2}, \chi_{1}}(16)=16 .
$$

From Table 3.3.1, we have

$$
b_{1}(16)=1, \quad b_{2}(16)=b_{3}(16)=0, \quad b_{4}(16)=-11 .
$$

Thus we have

$$
N(1,2,2,7 ; 16)=\frac{7}{4}(16)-\frac{1}{4}+\frac{7}{4}-\frac{1}{4}(16)-\frac{7}{8}-\frac{1}{8}(-11)=26,
$$

which agrees with the value of $N(1,2,2,7 ; 16)$ in Table 3.3.12. 


\subsection{The space $M_{2}\left(\Gamma_{0}(56), \chi_{5}\right)$}

Let $\chi_{0}$ be the trivial character and $\chi_{5}$ as in (3.1.4). We define the Eisenstein series

$$
\begin{aligned}
& E_{2, \chi_{5}, \chi_{0}}(q)=\sum_{n=1}^{\infty} \sigma_{\left(\chi_{5}, \chi_{0}\right)}(n) q^{n}, \\
& E_{2, \chi_{0}, \chi_{5}}(q)=-\frac{1}{2}+\sum_{n=1}^{\infty} \sigma_{\left(\chi_{0}, \chi_{5}\right)}(n) q^{n} .
\end{aligned}
$$

We determine $N\left(a_{1}, a_{2}, a_{3}, a_{4} ; n\right)$ for the quaternary quadratic forms listed in the third column of Table 3.1.1 in terms of $\sigma_{(\chi, \psi)}(n)$, where $\chi, \psi \in\left\{\chi_{0}, \chi_{5}\right\}$, and $c_{k}(n)(1 \leq$ $k \leq 6)$ defined by

$$
\begin{aligned}
& C_{1}(q)=\sum_{n=1}^{\infty} c_{1}(n) q^{n}=\frac{\eta^{3}(2 z) \eta(7 z) \eta^{2}(8 z) \eta(28 z)}{\eta(z) \eta^{2}(4 z)} \\
& C_{2}(q)=\sum_{n=1}^{\infty} c_{2}(n) q^{n}=\frac{\eta(2 z) \eta^{2}(7 z) \eta(8 z) \eta^{3}(28 z)}{\eta^{2}(14 z) \eta(56 z)} \\
& C_{3}(q)=\sum_{n=1}^{\infty} c_{3}(n) q^{n}=\frac{\eta^{2}(z) \eta^{3}(4 z) \eta(14 z) \eta(56 z)}{\eta^{2}(2 z) \eta(8 z)} \\
& C_{4}(q)=\sum_{n=1}^{\infty} c_{4}\left(n q^{n}\right)=\frac{\eta^{6}(2 z) \eta(8 z) \eta^{4}(28 z)}{\eta^{2}(z) \eta^{3}(4 z) \eta(14 z) \eta(56 z)} \\
& C_{5}(q)=\sum_{n=1}^{\infty} c_{5}(n) q^{n}=\frac{\eta^{4}(2 z) \eta(7 z) \eta^{6}(28 z)}{\eta(z) \eta(4 z) \eta^{3}(14 z) \eta^{2}(56 z)} \\
& C_{6}(q)=\sum_{n=1}^{\infty} c_{6}(n) q^{n}=\frac{\eta^{4}(4 z) \eta^{6}(14 z) \eta(56 z)}{\eta(2 z) \eta^{2}(7 z) \eta(8 z) \eta^{3}(28 z)} .
\end{aligned}
$$

There is no linear relationship among the $C_{k}(q), 1 \leq k \leq 6$. The first fifty-six values of $c_{k}(n), 1 \leq k \leq 6$, are given in Table 3.4.1.

Table 3.4.1

\begin{tabular}{r|rrrrrr|r|rrrrrr}
\hline$n$ & $c_{1}(n)$ & $c_{2}(n)$ & $c_{3}(n)$ & $c_{4}(n)$ & $c_{5}(n)$ & $c_{6}(n)$ & $n$ & $c_{1}(n)$ & $c_{2}(n)$ & $c_{3}(n)$ & $c_{4}(n)$ & $c_{5}(n)$ & $c_{6}(n)$ \\
\hline 1 & 0 & 1 & 0 & 0 & 1 & 0 & 29 & 0 & 2 & -2 & 0 & 4 & 4 \\
2 & 1 & 0 & 0 & 1 & 1 & 1 & 30 & 0 & 4 & -4 & 0 & 4 & 4 \\
3 & 1 & -1 & 1 & 2 & -2 & 0 & 31 & -4 & -4 & 0 & -4 & -8 & 4
\end{tabular}




\begin{tabular}{r|rrrrrr|r|rrrrrr}
4 & -1 & 0 & -2 & -1 & -1 & 1 & 32 & 3 & -2 & 2 & 5 & -5 & 3 \\
5 & 0 & -1 & 1 & -2 & 0 & 0 & 33 & 0 & 0 & -4 & 0 & -4 & 0 \\
6 & 1 & 0 & -2 & 2 & -2 & -2 & 34 & -2 & 0 & 0 & -6 & 2 & -6 \\
7 & 0 & 0 & 1 & 0 & 1 & 0 & 35 & -1 & 1 & -1 & 0 & 0 & -2 \\
8 & -1 & -2 & 2 & -3 & -1 & -1 & 36 & 1 & 0 & -2 & 5 & -7 & -5 \\
9 & -2 & -1 & 2 & -2 & -1 & 2 & 37 & 4 & -2 & 2 & 8 & -4 & 4 \\
10 & -1 & 2 & 0 & 0 & 4 & 0 & 38 & 1 & 4 & 2 & 2 & 6 & -2 \\
11 & 0 & 2 & -2 & 2 & 2 & 2 & 39 & -2 & 2 & 4 & -2 & 4 & 2 \\
12 & 0 & 2 & -2 & -2 & 2 & -2 & 40 & 2 & -4 & 0 & 4 & -4 & -4 \\
13 & -2 & 1 & -1 & -2 & 0 & -4 & 41 & 4 & 2 & 4 & 4 & 10 & -4 \\
14 & 0 & 0 & 0 & 1 & -1 & 1 & 42 & -1 & -2 & 0 & -2 & -2 & 2 \\
15 & 2 & 2 & 0 & 2 & 4 & -2 & 43 & -4 & 2 & -2 & -6 & 2 & -6 \\
16 & -1 & 2 & 2 & 1 & 3 & -1 & 44 & -2 & 0 & 0 & -2 & 2 & -6 \\
17 & 0 & -2 & -4 & 0 & -6 & 0 & 45 & 2 & -5 & 5 & 2 & -8 & -4 \\
18 & 1 & -4 & 4 & 3 & -5 & -1 & 46 & -4 & 4 & -4 & -8 & 4 & -4 \\
19 & -1 & -1 & 1 & -2 & -2 & -4 & 47 & 0 & 0 & 0 & 0 & 0 & 0 \\
20 & 0 & -2 & 2 & 0 & -4 & 4 & 48 & -2 & 0 & 0 & -2 & 2 & 10 \\
21 & 0 & 1 & -1 & 0 & 2 & 2 & 49 & 0 & 1 & 0 & 0 & 1 & 0 \\
22 & 0 & -4 & 0 & -2 & -6 & 2 & 50 & -1 & 4 & -4 & 1 & 1 & 5 \\
23 & 2 & -2 & -4 & 2 & -4 & -2 & 51 & -2 & -2 & 2 & -4 & -4 & -8 \\
24 & 0 & 4 & 0 & -2 & 10 & 2 & 52 & 4 & 2 & -2 & 8 & 4 & 4 \\
25 & 2 & 1 & 2 & 2 & 5 & -2 & 53 & -4 & 4 & -4 & -4 & 4 & -4 \\
26 & 1 & 2 & 4 & 0 & 4 & 0 & 54 & -4 & -4 & -4 & -4 & -4 & 4 \\
27 & 2 & 0 & 0 & 0 & 4 & 8 & 55 & -4 & 4 & 0 & -4 & 0 & 4 \\
28 & 1 & -2 & 0 & 1 & -3 & -1 & 56 & -1 & 2 & -2 & -1 & 1 & -3 \\
\hline
\end{tabular}

Theorem 3.4.1. Let $\left(a_{1}, a_{2}, a_{3}, a_{4}\right)$ be as in the third column of Table 3.1.1.Then $\varphi\left(q^{a_{1}}\right) \varphi\left(q^{a_{2}}\right) \varphi\left(q^{a_{3}}\right) \varphi\left(q^{a_{4}}\right) \in M_{2}\left(\Gamma_{0}(56), \chi_{5}\right)$.

Proof. We have $N=56$. First we consider quadratic form $(1,2,7,7)$. By (2.1.9) we have

$$
\varphi(q) \varphi\left(q^{2}\right) \varphi^{2}\left(q^{7}\right)=\frac{\eta^{3}(2 z) \eta^{3}(4 z) \eta^{10}(14 z)}{\eta^{2}(z) \eta^{4}(7 z) \eta^{2}(8 z) \eta^{4}(28 z)}
$$

Then we have Table 3.4.2(a) and Table 3.4.2(b).

Table 3.4.2(a)

\begin{tabular}{r|rrrrrrr}
\hline$\delta$ & 1 & 2 & 4 & 7 & 8 & 14 & 28 \\
\hline$r_{\delta}$ & -2 & 3 & 3 & -4 & -2 & 10 & -4 \\
\hline
\end{tabular}

It can be seen from Table 3.4.2(a) that conditions (L1) and (L2) are satisfied. 
Table 3.4.2(b)

\begin{tabular}{r|rrrrrrrr}
\hline$d \mid 56$ & 1 & 2 & 4 & 7 & 8 & 14 & 28 & 56 \\
\hline$\sum_{1 \leq \delta \mid 56} \frac{\operatorname{gcd}(d, \delta)^{2} \cdot r_{\delta}}{\delta}$ & 0 & $54 / 7$ & 12 & 0 & 0 & 90 & 12 & 0 \\
\hline
\end{tabular}

From Table 3.4.2(b) the condition (L3) is also satisfied. Thus $\varphi(q) \varphi\left(q^{2}\right) \varphi^{2}\left(q^{7}\right) \in$ $M_{2}\left(\Gamma_{0}(56), \chi_{5}\right)$. Secondly we consider the form $(1,1,7,14)$. By $(2.1 .9)$ we have

$$
\varphi^{2}(q) \varphi\left(q^{7}\right) \varphi\left(q^{14}\right)=\frac{\eta^{10}(2 z) \eta^{3}(14 z) \eta^{3}(28 z)}{\eta^{4}(z) \eta^{4}(4 z) \eta^{2}(7 z) \eta^{2}(56 z)}
$$

Then we have

Table 3.4.3(a)

\begin{tabular}{r|rrrrrrr}
\hline$\delta$ & 1 & 2 & 4 & 7 & 14 & 28 & 56 \\
\hline$r_{\delta}$ & -4 & 10 & -4 & -2 & 3 & 3 & -2 \\
\hline
\end{tabular}

It can be seen from Table 3.4.3(a) that conditions (L1) and (L2) are satisfied.

Table 3.4.3(b)

\begin{tabular}{r|rrrrrrrr}
\hline$d \mid 56$ & 1 & 2 & 4 & 7 & 8 & 14 & 28 & 56 \\
\hline$\sum_{1 \leq \delta \mid 56} \frac{\operatorname{gcd}(d, \delta)^{2} \cdot r_{\delta}}{\delta}$ & 0 & $90 / 7$ & $12 / 7$ & 0 & 0 & 54 & 84 & 0 \\
\hline
\end{tabular}

From Table 3.4.3(b) the condition (L3) is also satisfied. Thus $\varphi^{2}(q) \varphi\left(q^{7}\right) \varphi\left(q^{14}\right) \in$ $M_{2}\left(\Gamma_{0}(56), \chi_{5}\right)$. Thirdly we consider the form $(2,2,7,14)$. We have

$$
\varphi^{2}\left(q^{2}\right) \varphi\left(q^{7}\right) \varphi\left(q^{14}\right)=\frac{\eta^{10}(4 z) \eta^{3}(14 z) \eta^{3}(28 z)}{\eta^{4}(2 z) \eta^{2}(7 z) \eta^{4}(8 z) \eta^{2}(56 z)}
$$

Then we have

Table 3.4.4(a)

\begin{tabular}{r|rrrrrrr}
\hline$\delta$ & 2 & 4 & 7 & 8 & 14 & 28 & 56 \\
\hline$r_{\delta}$ & -4 & 10 & -2 & -4 & 3 & 3 & -2 \\
\hline
\end{tabular}

It can be seen from Table 3.4.4(a) that conditions $(L 1)$ and $(L 2)$ are satisfied. 
Table 3.4.4(b)

\begin{tabular}{r|rrrrrrrr}
\hline$d \mid 56$ & 1 & 2 & 4 & 7 & 8 & 14 & 28 & 56 \\
\hline$\sum_{1 \leq \delta \mid 56} \frac{\operatorname{gcd}(d, \delta)^{2} \cdot r_{\delta}}{\delta}$ & 0 & $6 / 7$ & $180 / 7$ & 0 & 0 & 42 & 108 & 0 \\
\hline
\end{tabular}

From Table 3.4.4(b) the condition (L3) is also satisfied. Thus $\varphi^{2}\left(q^{2}\right) \varphi\left(q^{7}\right) \varphi\left(q^{14}\right) \in$ $M_{2}\left(\Gamma_{0}(56), \chi_{5}\right)$. Fourthly we consider the form $(1,2,14,14)$. We have

$$
\varphi(q) \varphi\left(q^{2}\right) \varphi^{2}\left(q^{14}\right)=\frac{\eta^{3}(2 z) \eta^{3}(4 z) \eta^{10}(28 z)}{\eta^{2}(z) \eta^{2}(8 z) \eta^{4}(14 z) \eta^{4}(56 z)}
$$

Then we have

Table 3.4.5(a)

\begin{tabular}{r|rrrrrrr}
\hline$\delta$ & 1 & 2 & 4 & 8 & 14 & 28 & 56 \\
\hline$r_{\delta}$ & -2 & 3 & 3 & -2 & -4 & 10 & -4 \\
\hline
\end{tabular}

It can be seen from Table 3.4.5(a) that conditions (L1) and (L2) are satisfied.

Table 3.4.5(b)

\begin{tabular}{r|rrrrrrrr}
\hline$d \mid 56$ & 1 & 2 & 4 & 7 & 8 & 14 & 28 & 56 \\
\hline$\sum_{1 \leq \delta \mid 56} \frac{\operatorname{gcd}(d, \delta)^{2} \cdot r_{\delta}}{\delta}$ & 0 & 6 & $108 / 7$ & 0 & 0 & 6 & 180 & 0 \\
\hline
\end{tabular}

From Table 3.4.5(b) the condition (L3) is also satisfied. Thus $\varphi(q) \varphi\left(q^{2}\right) \varphi^{2}\left(q^{14}\right) \in$ $M_{2}\left(\Gamma_{0}(56), \chi_{5}\right)$.

Theorem 3.4.2. $C_{k}(q)(1 \leq k \leq 6)$ given by (3.4.3)-(3.4.8) are in $S_{2}\left(\Gamma_{0}(56), \chi_{5}\right)$.

Proof. We will check conditions (L1), (L2) and (L4) of Theorem 3.1.1. We have $N=56$. First we consider

$$
C_{1}(q)=\frac{\eta^{3}(2 z) \eta(7 z) \eta^{2}(8 z) \eta(28 z)}{\eta(z) \eta^{2}(4 z)}
$$

Then we have 
Table 3.4.6(a)

\begin{tabular}{r|rrrrrr}
\hline$\delta$ & 1 & 2 & 4 & 7 & 8 & 28 \\
\hline$r_{\delta}$ & -1 & 3 & -2 & 1 & 2 & 1 \\
\hline
\end{tabular}

It can be seen from Table 3.4.6(a) that conditions $(L 1)$ and $(L 2)$ are satisfied.

Table 3.4.6(b)

\begin{tabular}{r|rrrrrrrr}
\hline$d \mid 56$ & 1 & 2 & 4 & 7 & 8 & 14 & 28 & 56 \\
\hline$\sum_{1 \leq \delta \mid 56} \frac{\operatorname{gcd}(d, \delta)^{2} \cdot r_{\delta}}{\delta}$ & $3 / 7$ & $30 / 7$ & $12 / 7$ & 9 & $96 / 7$ & 18 & 36 & 48 \\
\hline
\end{tabular}

From Table 3.4.6(b) the condition $(L 4)$ is also satisfied. Thus $C_{1}(q) \in S_{2}\left(\Gamma_{0}(56), \chi_{5}\right)$.

Secondly we consider

$$
C_{2}(q)=\frac{\eta(2 z) \eta^{2}(7 z) \eta(8 z) \eta^{3}(28 z)}{\eta^{2}(14 z) \eta(56 z)}
$$

Then we have

Table 3.4.7(a)

\begin{tabular}{r|rrrrrr}
\hline$\delta$ & 2 & 7 & 8 & 14 & 28 & 56 \\
\hline$r_{\delta}$ & 1 & 2 & 1 & -2 & 3 & -1 \\
\hline
\end{tabular}

It can be seen from Table 3.4.7(a) that conditions (L1) and (L2) are satisfied.

Table 3.4.7(b)

\begin{tabular}{r|rrrrrrrr}
\hline$d \mid 56$ & 1 & 2 & 4 & 7 & 8 & 14 & 28 & 56 \\
\hline$\sum_{1 \leq \delta \mid 56} \frac{\operatorname{gcd}(d, \delta)^{2} \cdot r_{\delta}}{\delta}$ & $6 / 7$ & $18 / 7$ & $36 / 7$ & 12 & $72 / 7$ & 6 & 60 & 24 \\
\hline
\end{tabular}

From Table 3.4.7(b) the condition ( $L 4)$ is also satisfied. Thus $C_{2}(q) \in S_{2}\left(\Gamma_{0}(56), \chi_{5}\right)$.

Thirdly we consider

$$
C_{3}(q)=\frac{\eta^{2}(z) \eta^{3}(4 z) \eta(14 z) \eta(56 z)}{\eta^{2}(2 z) \eta(8 z)}
$$

Then we have 
Table 3.4.8(a)

\begin{tabular}{r|rrrrrr}
\hline$\delta$ & 1 & 2 & 4 & 8 & 14 & 56 \\
\hline$r_{\delta}$ & 2 & -2 & 3 & -1 & 1 & 1 \\
\hline
\end{tabular}

It can be seen from Table 3.4.8(a) that conditions $(L 1)$ and $(L 2)$ are satisfied.

Table 3.4.8(b)

\begin{tabular}{r|rrrrrrrr}
\hline$d \mid 56$ & 1 & 2 & 4 & 7 & 8 & 14 & 28 & 56 \\
\hline$\sum_{1 \leq \delta \mid 56} \frac{\operatorname{gcd}(d, \delta)^{2} \cdot r_{\delta}}{\delta}$ & $12 / 7$ & $6 / 7$ & $60 / 7$ & 6 & $24 / 7$ & 18 & 36 & 72 \\
\hline
\end{tabular}

From Table 3.4.8(b) the condition $(L 4)$ is also satisfied. Thus $C_{3}(q) \in S_{2}\left(\Gamma_{0}(56), \chi_{5}\right)$. Fourthly we consider

$$
C_{4}(q)=\frac{\eta^{6}(2 z) \eta(8 z) \eta^{4}(28 z)}{\eta^{2}(z) \eta^{3}(4 z) \eta(14 z) \eta(56 z)}
$$

Then we have

Table 3.4.9(a)

\begin{tabular}{r|rrrrrrr}
\hline$\delta$ & 1 & 2 & 4 & 8 & 14 & 28 & 56 \\
\hline$r_{\delta}$ & -2 & 6 & -3 & 1 & -1 & 4 & -1 \\
\hline
\end{tabular}

It can be seen from Table 3.4.9(a) that conditions $(L 1)$ and $(L 2)$ are satisfied.

\begin{tabular}{r|rrrrrrrr}
\multicolumn{1}{c}{ Table 3.4.9(b) } \\
\hline$d \mid 56$ & 1 & 2 & 4 & 7 & 8 & 14 & 28 & 56 \\
\hline$\sum_{1 \leq \delta \mid 56} \frac{\operatorname{gcd}(d, \delta)^{2} \cdot r_{\delta}}{\delta}$ & $3 / 7$ & $54 / 7$ & $12 / 7$ & 3 & $48 / 7$ & 18 & 84 & 48 \\
\hline
\end{tabular}

From Table 3.4.9(b) the condition $(L 4)$ is also satisfied. Thus $C_{4}(q) \in S_{2}\left(\Gamma_{0}(56), \chi_{5}\right)$.

Fifthly we consider

$$
C_{5}(q)=\frac{\eta^{4}(2 z) \eta(7 z) \eta^{6}(28 z)}{\eta(z) \eta(4 z) \eta^{3}(14 z) \eta^{2}(56 z)}
$$

Then we have 
Table 3.4.10(a)

\begin{tabular}{r|rrrrrrr}
\hline$\delta$ & 1 & 2 & 4 & 7 & 14 & 28 & 56 \\
\hline$r_{\delta}$ & -1 & 4 & -1 & 1 & -3 & 6 & -2 \\
\hline
\end{tabular}

It can be seen from Table 3.4.10(a) that conditions $(L 1)$ and $(L 2)$ are satisfied.

Table 3.4.10(b)

\begin{tabular}{r|rrrrrrrr}
\hline$d \mid 56$ & 1 & 2 & 4 & 7 & 8 & 14 & 28 & 56 \\
\hline$\sum_{1 \leq \delta \mid 56} \frac{\operatorname{gcd}(d, \delta)^{2} \cdot r_{\delta}}{\delta}$ & $6 / 7$ & 6 & $36 / 7$ & 6 & $24 / 7$ & 6 & 108 & 24 \\
\hline
\end{tabular}

From Table 3.4.10(b) the condition $(L 4)$ is also satisfied. Thus $C_{5}(q) \in S_{2}\left(\Gamma_{0}(56), \chi_{5}\right)$. Sixthly we consider

$$
C_{6}(q)=\frac{\eta^{4}(4 z) \eta^{6}(14 z) \eta(56 z)}{\eta(2 z) \eta^{2}(7 z) \eta(8 z) \eta^{3}(28 z)}
$$

Then we have

Table 3.4.11(a)

\begin{tabular}{r|rrrrrrr}
\hline$\delta$ & 2 & 4 & 7 & 8 & 14 & 28 & 56 \\
\hline$r_{\delta}$ & -1 & 4 & -2 & -1 & 6 & -3 & 1 \\
\hline
\end{tabular}

It can be seen from Table 3.4.11(a) that conditions (L1) and (L2) are satisfied.

Table 3.4.11(b)

\begin{tabular}{r|rrrrrrrr}
\hline$d \mid 56$ & 1 & 2 & 4 & 7 & 8 & 14 & 28 & 56 \\
\hline$\sum_{1 \leq \delta \mid 56} \frac{\operatorname{gcd}(d, \delta)^{2} \cdot r_{\delta}}{\delta}$ & $3 / 7$ & $18 / 7$ & 12 & 3 & $48 / 7$ & 54 & 12 & 48 \\
\hline
\end{tabular}

From Table 3.4.11(b) the condition $(L 4)$ is also satisfied. Thus $C_{6}(q) \in$ $S_{2}\left(\Gamma_{0}(56), \chi_{5}\right)$.

Theorem 3.4.3. (a) $\left\{C_{1}(q), \ldots, C_{6}(q)\right\}$ is a basis for $S_{2}\left(\Gamma_{0}(56), \chi_{5}\right)$.

(b) $\left\{E_{2, \chi_{5}, \chi_{0}}\left(q^{t}\right), E_{2, \chi_{0}, \chi_{5}}\left(q^{t}\right) \mid t=1,7\right\}$ is a basis for $E_{2}\left(\Gamma_{0}(56), \chi_{5}\right)$. 
(c) $\left\{E_{2, \chi_{5}, \chi_{0}}\left(q^{t}\right), E_{2, \chi_{0}, \chi_{5}}\left(q^{t}\right) \mid t=1,7\right\}$ together with $C_{k}(q)(1 \leq k \leq 6)$ constitute a basis for $M_{2}\left(\Gamma_{0}(56), \chi_{5}\right)$.

Proof. (a) By Theorem 3.4.2, $C_{k}(q)(1 \leq k \leq 6) \in S_{2}\left(\Gamma_{0}(56), \chi_{5}\right)$. There is no linear relationship among them. By Example 2.3.3, we have $\operatorname{dim} S_{2}\left(\Gamma_{0}(56), \chi_{5}\right)=6$. Thus $C_{k}(q)(1 \leq k \leq 6)$ constitute a basis for $S_{2}\left(\Gamma_{0}(56), \chi_{5}\right)$.

(b) By Example 2.3.3, we have $\operatorname{dim} E_{2}\left(\Gamma_{0}(56), \chi_{5}\right)=4$. By Theorem 2.2.3, $\left\{E_{2, \chi_{5}, \chi_{0}}\left(q^{t}\right)\right.$, $\left.E_{2, \chi_{0}, \chi_{5}}\left(q^{t}\right) \mid t=1,7\right\}$ constitute a basis for $E_{2}\left(\Gamma_{0}(56), \chi_{5}\right)$.

(c) By Example 2.3.3, we have $\operatorname{dim} M_{2}\left(\Gamma_{0}(56), \chi_{5}\right)=10$. Therefore, by (2.1.1) $\left\{E_{2, \chi_{5}, \chi_{0}}\left(q^{t}\right), E_{2, \chi_{0}, \chi_{5}}\left(q^{t}\right) \mid t=1,7\right\}$ together with $C_{k}(q)(1 \leq k \leq 6)$ constitute a basis for $M_{2}\left(\Gamma_{0}(56), \chi_{5}\right)$.

Theorem 3.4.4. Let $\chi_{0}$ be the trivial character and $\chi_{5}$ be as in (3.1.4). Then

$$
\begin{aligned}
\text { (a) } \varphi(q) \varphi\left(q^{2}\right) \varphi^{2}\left(q^{7}\right)= & \frac{4}{3} E_{2, \chi_{5}, \chi_{0}}(q)-\frac{28}{3} E_{2, \chi_{5}, \chi_{0}}\left(q^{7}\right)+\frac{1}{3} E_{2, \chi_{0}, \chi_{5}}(q)-\frac{7}{3} E_{2, \chi_{0}, \chi_{5}}\left(q^{7}\right) \\
& -4 C_{1}(q)-\frac{2}{3} C_{2}(q)+\frac{4}{3} C_{3}(q)+3 C_{4}(q)+C_{5}(q)-C_{6}(q), \\
\text { (b) } \varphi^{2}(q) \varphi\left(q^{7}\right) \varphi\left(q^{14}\right)= & \frac{4}{3} E_{2, \chi_{5}, \chi_{0}}(q)-\frac{28}{3} E_{2, \chi_{5}, \chi_{0}}\left(q^{7}\right)+\frac{1}{3} E_{2, \chi_{0}, \chi_{5}}(q)-\frac{7}{3} E_{2, \chi_{0}, \chi_{5}}\left(q^{7}\right) \\
& +4 C_{1}(q)+\frac{10}{3} C_{2}(q)+\frac{4}{3} C_{3}(q)-3 C_{4}(q)-C_{5}(q)+C_{6}(q), \\
& -2 C_{1}(q)-3 C_{2}(q)-C_{3}(q)+\frac{5}{3} C_{4}(q)+2 C_{5}(q)+\frac{2}{3} C_{6}(q), \\
\text { (c) } \varphi^{2}\left(q^{2}\right) \varphi\left(q^{7}\right) \varphi\left(q^{14}\right)= & \frac{2}{3} E_{2, \chi_{5}, \chi_{0}}(q)-\frac{14}{3} E_{2, \chi_{5}, \chi_{0}}\left(q^{7}\right)+\frac{1}{3} E_{2, \chi_{0}, \chi_{5}}(q)-\frac{7}{3} E_{2, \chi_{0}, \chi_{5}}\left(q^{7}\right) \\
\text { (d) } \varphi(q) \varphi\left(q^{2}\right) \varphi^{2}\left(q^{14}\right)= & \frac{2}{3} E_{2, \chi_{5}, \chi_{0}}(q)-\frac{14}{3} E_{2, \chi_{5}, \chi_{0}}\left(q^{7}\right)+\frac{1}{3} E_{2, \chi_{0}, \chi_{5}}(q)-\frac{7}{3} E_{2, \chi_{0}, \chi_{5}}\left(q^{7}\right) \\
& +2 C_{1}(q)+3 C_{2}(q)+C_{3}(q)-\frac{1}{3} C_{4}(q)-2 C_{5}(q)+\frac{2}{3} C_{6}(q) .
\end{aligned}
$$

Proof. Let $\left(a_{1}, a_{2}, a_{3}, a_{4}\right)$ be one of the quadratic forms listed in the third column of Table 3.1.1. By Theorem 3.4.1, $\varphi\left(q^{a_{1}}\right) \varphi\left(q^{a_{2}}\right) \varphi\left(q^{a_{3}}\right) \varphi\left(q^{a_{4}}\right) \in\left(M_{2}\left(\Gamma_{0}(56), \chi_{5}\right)\right.$. Therefore by Theorem 3.4.3 (c), $\varphi\left(q^{a_{1}}\right) \varphi\left(q^{a_{2}}\right) \varphi\left(q^{a_{3}}\right) \varphi\left(q^{a_{4}}\right)$ must be a linear combina- 
tion of $\left\{E_{2, \chi_{5}, \chi_{0}}\left(q^{t}\right), E_{2, \chi_{0}, \chi_{5}}\left(q^{t}\right) \mid t=1,7\right\}$ and $C_{k}(q)(1 \leq k \leq 6)$, namely

$$
\begin{aligned}
\varphi\left(q^{a_{1}}\right) \varphi\left(q^{a_{2}}\right) \varphi\left(q^{a_{3}}\right) \varphi\left(q^{a_{4}}\right)= & x_{1} E_{2, \chi_{5}, \chi_{0}}(q)+x_{2} E_{2, \chi_{5}, \chi_{0}}\left(q^{7}\right)+x_{3} E_{2, \chi_{0}, \chi_{5}}(q) \\
& +x_{4} E_{2, \chi_{0}, \chi_{5}}\left(q^{7}\right)+y_{1} C_{1}(q)+y_{2} C_{2}(q)+y_{3} C_{3}(q)+y_{4} C_{4}(q) \\
& +y_{5} C_{5}(q)+y_{6} C_{6}(q) .
\end{aligned}
$$

We equate the first fourteen coefficients of $q^{n}$ on both sides of the equation above to obtain a system of linear equations with the unknowns $x_{1}, x_{2}, x_{3}, x_{4}, y_{1}, y_{2}, y_{3}, y_{4}, y_{5}, y_{6}$. Then, using MAPLE we solve the system to find the asserted coefficients.

Theorem 3.4.5. Let $n \in \mathbb{N}$. Let $\sigma_{\chi_{i}, \chi_{j}}(n)$ be as in (2.2.1) for $i, j \in\{0,5\}$. Then

(a) $N(1,2,7,7 ; n)=\frac{4}{3} \sigma_{\chi_{5}, \chi_{0}}(n)-\frac{28}{3} \sigma_{\chi_{5}, \chi_{0}}(n / 7)+\frac{1}{3} \sigma_{\chi_{0}, \chi_{5}}(n)-\frac{7}{3} \sigma_{\chi_{0}, \chi_{5}}(n / 7)$

$$
-4 c_{1}(n)-\frac{2}{3} c_{2}(n)+\frac{4}{3} c_{3}(n)+3 c_{4}(n)+c_{5}(n)-c_{6}(n),
$$

(b) $N(1,1,7,14 ; n)=\frac{4}{3} \sigma_{\chi_{5}, \chi_{0}}(n)-\frac{28}{3} \sigma_{\chi_{5}, \chi_{0}}(n / 7)+\frac{1}{3} \sigma_{\chi_{0}, \chi_{5}}(n)-\frac{7}{3} \sigma_{\chi_{0}, \chi_{5}}(n / 7)$

$$
+4 c_{1}(n)+\frac{10}{3} c_{2}(n)+\frac{4}{3} c_{3}(n)-3 c_{4}(n)-c_{5}(n)+c_{6}(n)
$$

(c) $N(2,2,7,14 ; n)=\frac{2}{3} \sigma_{\chi_{5}, \chi_{0}}(n)-\frac{14}{3} \sigma_{\chi_{5}, \chi_{0}}(n / 7)+\frac{1}{3} \sigma_{\chi_{0}, \chi_{5}}(n)-\frac{7}{3} \sigma_{\chi_{0}, \chi_{5}}(n / 7)$

$$
-2 c_{1}(n)-3 c_{2}(n)-c_{3}(n)+\frac{5}{3} c_{4}(n)+2 c_{5}(n)+\frac{2}{3} c_{6}(n),
$$

(d) $N(1,2,14,14 ; n)=\frac{2}{3} \sigma_{\chi_{5}, \chi_{0}}(n)-\frac{14}{3} \sigma_{\chi_{5}, \chi_{0}}(n / 7)+\frac{1}{3} \sigma_{\chi_{0}, \chi_{5}}(n)-\frac{7}{3} \sigma_{\chi_{0}, \chi_{5}}(n / 7)$

$$
+2 c_{1}(n)+3 c_{2}(n)+c_{3}(n)-\frac{1}{3} c_{4}(n)-2 c_{5}(n)+\frac{2}{3} c_{6}(n) .
$$


Proof. Appealing to (2.1.6), (3.4.1), (3.4.2) and Theorem 3.4.4, we obtain

(a)

$\sum_{n=0}^{\infty} N(1,2,7,7 ; n) q^{n}=\varphi(q) \varphi\left(q^{2}\right) \varphi^{2}\left(q^{7}\right)$

$=\frac{4}{3} E_{2, \chi_{5}, \chi_{0}}(q)-\frac{28}{3} E_{2, \chi_{5}, \chi_{0}}\left(q^{7}\right)+\frac{1}{3} E_{2, \chi_{0}, \chi_{5}}(q)-\frac{7}{3} E_{2, \chi_{0}, \chi_{5}}\left(q^{7}\right)-4 C_{1}(q)$

$-\frac{2}{3} C_{2}(q)+\frac{4}{3} C_{3}(q)+3 C_{4}(q)+C_{5}(q)-C_{6}(q)$,

$=1+\sum_{n=1}^{\infty}\left(\frac{4}{3} \sigma_{\chi_{5}, \chi_{0}}(n)-\frac{28}{3} \sigma_{\chi_{5}, \chi_{0}}(n / 7)+\frac{1}{3} \sigma_{\chi_{0}, \chi_{5}}(n)-\frac{7}{3} \sigma_{\chi_{0}, \chi_{5}}(n / 7)\right.$

$\left.-4 c_{1}(n)-\frac{2}{3} c_{2}(n)+\frac{4}{3} c_{3}(n)+3 c_{4}(n)+c_{5}(n)-c_{6}(n)\right) q^{n}$,

(b) $\sum_{n=0}^{\infty} N(1,1,7,14 ; n) q^{n}=\varphi^{2}(q) \varphi\left(q^{7}\right) \varphi\left(q^{14}\right)$

$=\frac{4}{3} E_{2, \chi_{5}, \chi_{0}}(q)-\frac{28}{3} E_{2, \chi_{5}, \chi_{0}}\left(q^{7}\right)+\frac{1}{3} E_{2, \chi_{0}, \chi_{5}}(q)-\frac{7}{3} E_{2, \chi_{0}, \chi_{5}}\left(q^{7}\right)+4 C_{1}(q)$

$+\frac{10}{3} C_{2}(q)+\frac{4}{3} C_{3}(q)-3 C_{4}(q)-C_{5}(q)+C_{6}(q)$,

$=1+\sum_{n=1}^{\infty}\left(\frac{4}{3} \sigma_{\chi_{5}, \chi_{0}}(n)-\frac{28}{3} \sigma_{\chi_{5}, \chi_{0}}(n / 7)+\frac{1}{3} \sigma_{\chi_{0}, \chi_{5}}(n)-\frac{7}{3} \sigma_{\chi_{0}, \chi_{5}}(n / 7)\right.$

$\left.+4 c_{1}(n)+\frac{10}{3} c_{2}(n)+\frac{4}{3} c_{3}(n)-3 c_{4}(n)-c_{5}(n)+c_{6}(n)\right) q^{n}$,

(c) $\sum_{n=0}^{\infty} N(2,2,7,14 ; n) q^{n}=\varphi^{2}\left(q^{2}\right) \varphi\left(q^{7}\right) \varphi\left(q^{14}\right)$

$=\frac{2}{3} E_{2, \chi_{5}, \chi_{0}}(q)-\frac{14}{3} E_{2, \chi_{5}, \chi_{0}}\left(q^{7}\right)+\frac{1}{3} E_{2, \chi_{0}, \chi_{5}}(q)-\frac{7}{3} E_{2, \chi_{0}, \chi_{5}}\left(q^{7}\right)$

$-2 C_{1}(q)-3 C_{2}(q)-C_{3}(q)+\frac{5}{3} C_{4}(q)+2 C_{5}(q)+\frac{2}{3} C_{6}(q)$,

$=1+\sum_{n=1}^{\infty}\left(\frac{2}{3} \sigma_{\chi_{5}, \chi_{0}}(n)-\frac{14}{3} \sigma_{\chi_{5}, \chi_{0}}(n / 7)+\frac{1}{3} \sigma_{\chi_{0}, \chi_{5}}(n)-\frac{7}{3} \sigma_{\chi_{0}, \chi_{5}}(n / 7)\right.$

$\left.-2 c_{1}(n)-3 c_{2}(n)-c_{3}(n)+\frac{5}{3} c_{4}(n)+2 c_{5}(n)+\frac{2}{3} c_{6}(n)\right) q^{n}$, 


$$
\text { (d) } \begin{aligned}
& \sum_{n=0}^{\infty} N(1,2,14,14 ; n) q^{n}=\varphi(q) \varphi\left(q^{2}\right) \varphi^{2}\left(q^{14}\right) \\
&= \frac{2}{3} E_{2, \chi_{5}, \chi_{0}}(q)-\frac{14}{3} E_{2, \chi_{5}, \chi_{0}}\left(q^{7}\right)+\frac{1}{3} E_{2, \chi_{0}, \chi_{5}}(q)-\frac{7}{3} E_{2, \chi_{0}, \chi_{5}}\left(q^{7}\right) \\
&+2 C_{1}(q)+3 C_{2}(q)+C_{3}(q)-\frac{1}{3} C_{4}(q)-2 C_{5}(q)+\frac{2}{3} C_{6}(q), \\
&= 1+\sum_{n=1}^{\infty}\left(\frac{2}{3} \sigma_{\chi_{5}, \chi_{0}}(n)-\frac{14}{3} \sigma_{\chi_{5}, \chi_{0}}(n / 7)+\frac{1}{3} \sigma_{\chi_{0}, \chi_{5}}(n)-\frac{7}{3} \sigma_{\chi_{0}, \chi_{5}}(n / 7)\right. \\
&\left.+2 c_{1}(n)+3 c_{2}(n)+c_{3}(n)-\frac{1}{3} c_{4}(n)-2 c_{5}(n)+\frac{2}{3} c_{6}(n)\right) q^{n} .
\end{aligned}
$$

Equating the coefficients of $q^{n}$ on both sides of equations (a)-(d) yields the results.

The values of $N\left(a_{1}, a_{2}, a_{3}, a_{4} ; n\right)$ for $1 \leq n \leq 20$ for the quadratic forms $\left(a_{1}, a_{2}, a_{3}, a_{4}\right)$ in Theorem 3.4.5 are given in Table 3.4.12.

Table 3.4 .12

\begin{tabular}{l|rrrrrrrrrrrrrrrrrrrr}
\hline \multicolumn{1}{c|}{$n$} & 1 & 2 & 3 & 4 & 5 & 6 & 7 & 8 & 9 & 10 & 11 & 12 & 13 & 14 & 15 & 16 & 17 & 18 & 19 & 20 \\
\hline$N(1,2,7,7 ; n)$ & 2 & 2 & 4 & 2 & 0 & 4 & 4 & 10 & 14 & 16 & 12 & 4 & 16 & 4 & 16 & 34 & 20 & 30 & 20 & 16 \\
$N(1,1,7,14 ; n)$ & 4 & 4 & 0 & 4 & 8 & 0 & 2 & 12 & 12 & 8 & 8 & 16 & 8 & 2 & 16 & 20 & 24 & 12 & 16 & 24 \\
$N(2,2,7,14 ; n)$ & 0 & 4 & 0 & 4 & 0 & 0 & 2 & 4 & 8 & 8 & 8 & 0 & 0 & 2 & 8 & 12 & 16 & 12 & 0 & 8 \\
$N(1,2,14,14 ; n)$ & 2 & 2 & 4 & 2 & 0 & 4 & 0 & 2 & 6 & 0 & 4 & 4 & 0 & 4 & 8 & 10 & 20 & 14 & 4 & 16 \\
\hline
\end{tabular}

Again one can verify the values in Table 3.4.12 by using (2.2.1), (3.1.4) and Table 3.4.1.

\subsection{The space $M_{2}\left(\Gamma_{0}(56), \chi_{6}\right)$}

Let $\chi_{0}$ be the trivial character and $\chi_{1}, \chi_{4}, \chi_{6}$ as in (3.1.3) and (3.1.4). We define the Eisenstein series

$$
E_{2, \chi_{4}, \chi_{1}}(q)=\sum_{n=1}^{\infty} \sigma_{\left(\chi_{4}, \chi_{1}\right)}(n) q^{n}
$$




$$
\begin{aligned}
& E_{2, \chi_{1}, \chi_{4}}(q)=\sum_{n=1}^{\infty} \sigma_{\left(\chi_{1}, \chi_{4}\right)}(n) q^{n} \\
& E_{2, \chi_{6}, \chi_{0}}(q)=\sum_{n=1}^{\infty} \sigma_{\left(\chi_{6}, \chi_{0}\right)}(n) q^{n} \\
& E_{2, \chi_{0}, \chi_{6}}(q)=-10+\sum_{n=1}^{\infty} \sigma_{\left(\chi_{0}, \chi_{6}\right)}(n) q^{n} .
\end{aligned}
$$

We determine the representation number for the quaternary quadratic forms listed in the fourth column of Table 3.1.1 in terms of $\sigma_{(\chi, \psi)}(n)$, where $\chi, \psi \in\left\{\chi_{0}, \chi_{1}, \chi_{4}, \chi_{6}\right\}$, and $d_{k}(n)(1 \leq k \leq 6)$ defined by

$$
\begin{aligned}
& D_{1}(q)=\sum_{n=1}^{\infty} d_{1}(n)=\frac{\eta^{4}(2 z) \eta^{2}(4 z) \eta(14 z) \eta(56 z)}{\eta^{2}(z) \eta(8 z) \eta(28 z)} \\
& D_{2}(q)=\sum_{n=1}^{\infty} d_{2}(n) q^{n}=\frac{\eta^{2}(2 z) \eta^{4}(4 z) \eta(7 z) \eta(28 z)}{\eta(z) \eta^{2}(8 z) \eta(14 z)} \\
& D_{3}(q)=\sum_{n=1}^{\infty} d_{3}(n) q^{n}=\frac{\eta^{3}(2 z) \eta(7 z) \eta^{2}(56 z)}{\eta(z) \eta(4 z)} \\
& D_{4}(q)=\sum_{n=1}^{\infty} d_{4}(n) q^{n}=\frac{\eta^{3}(4 z) \eta^{2}(7 z) \eta(56 z)}{\eta(2 z) \eta(8 z)} \\
& D_{5}(q)=\sum_{n=1}^{\infty} d_{5}(n) q^{n}=\frac{\eta(2 z) \eta(8 z) \eta^{4}(14 z) \eta^{2}(28 z)}{\eta(4 z) \eta^{2}(7 z) \eta(56 z)} \\
& D_{6}(q)=\sum_{n=1}^{\infty} d_{6}(n) q^{n}=\frac{\eta(z) \eta^{2}(8 z) \eta^{3}(14 z)}{\eta(7 z) \eta(28 z)} .
\end{aligned}
$$

There is no linear relationship among the $D_{k}(q), 1 \leq k \leq 6$. The first fifty-six values of $d_{k}(n), 1 \leq k \leq 6$, are given in Table 3.5.1.

Table 3.5.1

\begin{tabular}{r|rrrrrr|r|rrrrrr}
\hline$n$ & $d_{1}(n)$ & $d_{2}(n)$ & $d_{3}(n)$ & $d_{4}(n)$ & $d_{5}(n)$ & $d_{6}(n)$ & $n$ & $d_{1}(n)$ & $d_{2}(n)$ & $d_{3}(n)$ & $d_{4}(n)$ & $d_{5}(n)$ & $d_{6}(n)$ \\
\hline 1 & 0 & 1 & 0 & 0 & 0 & 1 & 29 & 8 & 12 & 0 & 0 & 0 & 0 \\
2 & 1 & 1 & 0 & 0 & 1 & -1 & 30 & 4 & -4 & 0 & 0 & 0 & 6 \\
3 & 2 & 0 & 0 & 1 & 0 & -1 & 31 & 4 & -8 & 2 & 2 & 0 & 0 \\
4 & 1 & 1 & 0 & 0 & -1 & 0 & 32 & -3 & 7 & 0 & 0 & -1 & 4
\end{tabular}




\begin{tabular}{r|rrrrrr|r|rrrrrr}
5 & 2 & -4 & 1 & 1 & 0 & 0 & 33 & 4 & 0 & 0 & 2 & 0 & -2 \\
6 & 0 & -4 & 1 & 0 & 0 & 1 & 34 & 0 & 8 & -2 & 0 & 0 & -2 \\
7 & -4 & 1 & -1 & -1 & 0 & 0 & 35 & -4 & 0 & 0 & -1 & 2 & -5 \\
8 & 1 & -5 & 0 & 0 & -1 & 2 & 36 & 7 & -5 & 0 & 0 & 1 & 0 \\
9 & -2 & 3 & 0 & 0 & 2 & -3 & 37 & -12 & -4 & 0 & 0 & -4 & 0 \\
10 & -4 & 4 & -1 & -2 & 0 & 1 & 38 & 0 & 4 & -1 & 0 & 0 & -1 \\
11 & 2 & -4 & 0 & 0 & -2 & 2 & 39 & 2 & -4 & 0 & 0 & 2 & 0 \\
12 & -4 & 8 & -2 & -2 & 0 & 0 & 40 & 0 & -8 & 2 & 0 & 0 & 2 \\
13 & -2 & 4 & -1 & -1 & 0 & 0 & 41 & 0 & 0 & 0 & 0 & 0 & 0 \\
14 & 1 & -1 & 1 & 2 & 1 & -2 & 42 & -8 & 8 & -1 & -2 & 0 & -5 \\
15 & -2 & 4 & 0 & 0 & -2 & 0 & 43 & -6 & 12 & 0 & 0 & 6 & -6 \\
16 & -5 & -3 & 0 & 0 & 1 & -4 & 44 & -8 & 4 & 0 & 0 & 0 & 0 \\
17 & -4 & 0 & 0 & -2 & 0 & 2 & 45 & -6 & 12 & -3 & -3 & 0 & 0 \\
18 & 3 & 7 & 0 & 0 & -1 & 3 & 46 & 2 & -2 & 0 & 0 & -2 & -4 \\
19 & -2 & 0 & 0 & -1 & 0 & 1 & 47 & -4 & 8 & -2 & -2 & 0 & 0 \\
20 & 4 & 0 & 0 & 2 & 0 & -2 & 48 & -8 & 0 & 0 & -4 & 0 & 4 \\
21 & 4 & -8 & 1 & 1 & 2 & 0 & 49 & 8 & -7 & 0 & 2 & -4 & 3 \\
22 & -4 & -8 & 0 & 0 & 0 & -2 & 50 & -5 & -9 & 0 & 0 & -1 & -1 \\
23 & 6 & 2 & 0 & 0 & 2 & 0 & 51 & 4 & 0 & 0 & 0 & -4 & 12 \\
24 & 8 & -8 & 2 & 4 & 0 & -2 & 52 & -4 & 0 & 0 & -2 & 0 & 2 \\
25 & 2 & -5 & 0 & 0 & -2 & 1 & 53 & -8 & -12 & 0 & 0 & 0 & 0 \\
26 & 4 & -4 & 1 & 2 & 0 & -1 & 54 & 0 & 0 & 0 & 0 & 0 & 0 \\
27 & 0 & 0 & 0 & 0 & 0 & 0 & 55 & 4 & -8 & 2 & 2 & 0 & 0 \\
28 & -1 & 7 & 0 & -2 & 1 & 4 & 56 & 7 & 9 & -2 & 0 & 1 & -4 \\
\hline
\end{tabular}

Theorem 3.5.1. Let $\left(a_{1}, a_{2}, a_{3}, a_{4}\right)$ be as in the fourth column of Table 3.1.1. Then $\varphi\left(q^{a_{1}}\right) \varphi\left(q^{a_{2}}\right) \varphi\left(q^{a_{3}}\right) \varphi\left(q^{a_{4}}\right) \in M_{2}\left(\Gamma_{0}(56), \chi_{6}\right)$.

Proof. We appeal to (2.1.9) for each quadratic forms and then check the conditions (L1), (L2) and (L3) of Theorem 3.1.1 for each quadratic form. We have $N=56$.

First we consider $(1,1,2,7)$

$$
\varphi^{2}(q) \varphi\left(q^{2}\right) \varphi\left(q^{7}\right)=\frac{\eta^{8}(2 z) \eta(4 z) \eta^{5}(14 z)}{\eta^{4}(z) \eta^{2}(7 z) \eta^{2}(8 z) \eta^{2}(28 z)}
$$

Then we have

Table 3.5.2(a)

\begin{tabular}{r|rrrrrrr}
\hline$\delta$ & 1 & 2 & 4 & 7 & 8 & 14 & 28 \\
\hline$r_{\delta}$ & -4 & 8 & 1 & -2 & -2 & 5 & -2 \\
\hline
\end{tabular}

It can be seen from Table 3.5.2(a) that conditions ( $L 1)$ and $(L 2)$ are satisfied. 
Table 3.5.2(b)

\begin{tabular}{r|rrrrrrrr}
\hline$d \mid 56$ & 1 & 2 & 4 & 7 & 8 & 14 & 28 & 56 \\
\hline$\sum_{1 \leq \delta \mid 56} \frac{\operatorname{gcd}(d, \delta)^{2} \cdot r_{\delta}}{\delta}$ & 0 & $90 / 7$ & 12 & 0 & 0 & 54 & 12 & 0 \\
\hline
\end{tabular}

From Table 3.5.2(b) the condition (L3) is also satisfied. Thus $\varphi^{2}(q) \varphi\left(q^{2}\right) \varphi\left(q^{7}\right) \in$ $M_{2}\left(\Gamma_{0}(56), \chi_{6}\right)$. Secondly we consider $(2,2,2,7)$. We have

$$
\varphi^{3}\left(q^{2}\right) \varphi\left(q^{7}\right)=\frac{\eta^{15}(4 z) \eta^{5}(14 z)}{\eta^{6}(2 z) \eta^{2}(7 z) \eta^{6}(8 z) \eta^{2}(28 z)}
$$

Then we have

Table 3.5.3(a)

\begin{tabular}{r|rrrrrr}
\hline$\delta$ & 2 & 4 & 7 & 8 & 14 & 28 \\
\hline$r_{\delta}$ & -6 & 15 & -2 & -6 & 5 & -2 \\
\hline
\end{tabular}

It can be seen from Table 3.5.3(a) that conditions $(L 1)$ and $(L 2)$ are satisfied.

Table 3.5.3(b)

\begin{tabular}{r|rrrrrrrr}
\hline$d \mid 56$ & 1 & 2 & 4 & 7 & 8 & 14 & 28 & 56 \\
\hline$\sum_{1 \leq \delta \mid 56} \frac{\operatorname{gcd}(d, \delta)^{2} \cdot r_{\delta}}{\delta}$ & 0 & $6 / 7$ & 36 & 0 & 0 & 42 & 36 & 0 \\
\hline
\end{tabular}

From Table 3.5.3(b) the condition (L3) is also satisfied. Thus $\varphi^{3}\left(q^{2}\right) \varphi\left(q^{7}\right) \in$ $M_{2}\left(\Gamma_{0}(56), \chi_{6}\right)$. Thirdly we consider $(2,7,7,7)$. We have

$$
\varphi\left(q^{2}\right) \varphi^{3}\left(q^{7}\right)=\frac{\eta^{5}(4 z) \eta^{15}(14 z)}{\eta^{2}(2 z) \eta^{6}(7 z) \eta^{2}(8 z) \eta^{6}(28 z)}
$$

Then we have

\begin{tabular}{r|rrrrrr}
\multicolumn{7}{c}{ Table 3.5.4(a) } \\
\hline$\delta$ & 2 & 4 & 7 & 8 & 14 & 28 \\
\hline$r_{\delta}$ & -2 & 5 & -6 & -2 & 15 & -6 \\
\hline
\end{tabular}

It can be seen from Table 3.5.4(a) that conditions (L1) and (L2) are satisfied. 
Table 3.5.4(b)

\begin{tabular}{r|rrrrrrrr}
\hline$d \mid 56$ & 1 & 2 & 4 & 7 & 8 & 14 & 28 & 56 \\
\hline$\sum_{1 \leq \delta \mid 56} \frac{\operatorname{gcd}(d, \delta)^{2} \cdot r_{\delta}}{\delta}$ & 0 & $18 / 7$ & 12 & 0 & 0 & 126 & 12 & 0 \\
\hline
\end{tabular}

From Table 3.5.4(b) the condition (L3) is also satisfied. Thus $\varphi\left(q^{2}\right) \varphi^{3}\left(q^{7}\right) \in$ $M_{2}\left(\Gamma_{0}(56), \chi_{6}\right)$. Fourthly we consider $(3,0,0,1)$. We have

$$
\varphi^{3}(q) \varphi\left(q^{14}\right)=\frac{\eta^{15}(2 z) \eta^{5}(28 z)}{\eta^{6}(z) \eta^{6}(4 z) \eta^{2}(14 z) \eta^{2}(56 z)}
$$

Then we have

Table 3.5.5(a)

\begin{tabular}{r|rrrrrr}
\hline$\delta$ & 1 & 2 & 4 & 14 & 28 & 56 \\
\hline$r_{\delta}$ & -6 & 15 & -6 & -2 & 5 & -2 \\
\hline
\end{tabular}

It can be seen from Table 3.5.5(a) that conditions (L1) and (L2) are satisfied.

Table 3.5.5(b)

\begin{tabular}{r|rrrrrrrr}
\hline$d \mid 56$ & 1 & 2 & 4 & 7 & 8 & 14 & 28 & 56 \\
\hline$\sum_{1 \leq \delta \mid 56} \frac{\operatorname{gcd}(d, \delta)^{2} \cdot r_{\delta}}{\delta}$ & 0 & 18 & $12 / 7$ & 0 & 0 & 18 & 84 & 0 \\
\hline
\end{tabular}

From Table 3.5.5(b) the condition (L3) is also satisfied. Thus $\varphi^{3}(q) \varphi\left(q^{14}\right) \in$ $M_{2}\left(\Gamma_{0}(56), \chi_{6}\right)$. Fifthly we consider $(1,2,2,14)$. We have

$$
\varphi(q) \varphi^{2}\left(q^{2}\right) \varphi\left(q^{14}\right)=\frac{\eta(2 z) \eta^{8}(4 z) \eta^{5}(28 z)}{\eta^{2}(z) \eta^{4}(8 z) \eta^{2}(14 z) \eta^{2}(56 z)}
$$

Then we have

Table 3.5.6(a)

\begin{tabular}{r|rrrrrrr}
\hline$\delta$ & 1 & 2 & 4 & 8 & 14 & 28 & 56 \\
\hline$r_{\delta}$ & -2 & 1 & 8 & -4 & -2 & 5 & -2 \\
\hline
\end{tabular}

It can be seen from Table 3.5.6(a) that conditions $(L 1)$ and $(L 2)$ are satisfied. 
Table 3.5.6(b)

\begin{tabular}{r|rrrrrrrr}
\hline$d \mid 56$ & 1 & 2 & 4 & 7 & 8 & 14 & 28 & 56 \\
\hline$\sum_{1 \leq \delta \mid 56} \frac{\operatorname{gcd}(d, \delta)^{2} \cdot r_{\delta}}{\delta}$ & 0 & 6 & $180 / 7$ & 0 & 0 & 6 & 108 & 0 \\
\hline
\end{tabular}

From Table 3.5.6(b) the condition (L3) is also satisfied. Thus $\varphi(q) \varphi^{2}\left(q^{2}\right) \varphi\left(q^{14}\right) \in$ $M_{2}\left(\Gamma_{0}(56), \chi_{6}\right)$. Sixthly we consider $(1,7,7,14)$. We have

$$
\varphi(q) \varphi^{2}\left(q^{7}\right) \varphi\left(q^{14}\right)=\frac{\eta^{5}(2 z) \eta^{8}(14 z) \eta(28 z)}{\eta^{2}(z) \eta^{2}(4 z) \eta^{4}(7 z) \eta^{2}(56 z)}
$$

Then we have

Table 3.5.7(a)

\begin{tabular}{r|rrrrrrr}
\hline$\delta$ & 1 & 2 & 4 & 7 & 14 & 28 & 56 \\
\hline$r_{\delta}$ & -2 & 5 & -2 & -4 & 8 & 1 & -2 \\
\hline
\end{tabular}

It can be seen from Table 3.5.7(a) that conditions $(L 1)$ and $(L 2)$ are satisfied.

Table 3.5.7(b)

\begin{tabular}{r|rrrrrrrr}
\hline$d \mid 56$ & 1 & 2 & 4 & 7 & 8 & 14 & 28 & 56 \\
\hline$\sum_{1 \leq \delta \mid 56} \frac{\operatorname{gcd}(d, \delta)^{2} \cdot r_{\delta}}{\delta}$ & 0 & $54 / 7$ & $12 / 7$ & 0 & 0 & 90 & 84 & 0 \\
\hline
\end{tabular}

From Table 3.5.7(b) the condition (L3) is also satisfied. Thus $\varphi(q) \varphi^{2}\left(q^{7}\right) \varphi\left(q^{14}\right) \in$ $M_{2}\left(\Gamma_{0}(56), \chi_{6}\right)$. Seventhly we consider $(2,7,14,14)$. Then

$$
\varphi\left(q^{2}\right) \varphi\left(q^{7}\right) \varphi^{2}\left(q^{14}\right)=\frac{\eta^{5}(4 z) \eta(14 z) \eta^{8}(28 z)}{\eta^{2}(2 z) \eta^{2}(7 z) \eta^{2}(8 z) \eta^{4}(56 z)}
$$

Then we have

Table 3.5.8(a)

\begin{tabular}{r|rrrrrrr}
\hline$\delta$ & 2 & 4 & 7 & 8 & 14 & 28 & 56 \\
\hline$r_{\delta}$ & -2 & 5 & -2 & -2 & 1 & 8 & -4 \\
\hline
\end{tabular}

It can be seen from Table 3.5.8(a) that conditions $(L 1)$ and $(L 2)$ are satisfied. 
Table 3.5.8(b)

\begin{tabular}{r|rrrrrrrr}
\hline$d \mid 56$ & 1 & 2 & 4 & 7 & 8 & 14 & 28 & 56 \\
\hline$\sum_{1 \leq \delta \mid 56} \frac{\operatorname{gcd}(d, \delta)^{2} \cdot r_{\delta}}{\delta}$ & 0 & $6 / 7$ & $108 / 7$ & 0 & 0 & 42 & 180 & 0 \\
\hline
\end{tabular}

From Table 3.5.8(b) the condition (L3) is also satisfied. Thus $\varphi(q) \varphi\left(q^{2}\right) \varphi\left(q^{7}\right) \varphi\left(q^{14}\right) \in$ $M_{2}\left(\Gamma_{0}(56), \chi_{6}\right)$. Eighthly we consider $(1,14,14,14)$. We have

$$
\varphi(q) \varphi^{3}\left(q^{14}\right)=\frac{\eta^{5}(2 z) \eta^{15}(28 z)}{\eta^{2}(z) \eta^{2}(4 z) \eta^{6}(14 z) \eta^{6}(56 z)}
$$

Then we have

Table 3.5.9(a)

\begin{tabular}{r|rrrrrr}
\hline$\delta$ & 1 & 2 & 4 & 14 & 28 & 56 \\
\hline$r_{\delta}$ & -2 & 5 & -2 & -6 & 15 & -6 \\
\hline
\end{tabular}

It can be seen from Table 3.5.9(a) that conditions $(L 1)$ and $(L 2)$ are satisfied.

Table 3.5.9(b)

\begin{tabular}{r|rrrrrrrr}
\hline$d \mid 56$ & 1 & 2 & 4 & 7 & 8 & 14 & 28 & 56 \\
\hline$\sum_{1 \leq \delta \mid 56} \frac{\operatorname{gcd}(d, \delta)^{2} \cdot r_{\delta}}{\delta}$ & 0 & 6 & $36 / 7$ & 0 & 0 & 6 & 252 & 0 \\
\hline
\end{tabular}

From Table 3.5.9(b) the condition (L3) is also satisfied. Thus $\varphi(q) \varphi^{3}\left(q^{14}\right) \in$ $M_{2}\left(\Gamma_{0}(56), \chi_{6}\right)$.

Theorem 3.5.2. $D_{k}(q)(1 \leq k \leq 6)$ given by $(3.5 .5)-(3.5 .10)$ are in $S_{2}\left(\Gamma_{0}(56), \chi_{6}\right)$.

Proof. We will check conditions (L1), (L2) and (L4) of Theorem 3.1.1. We have $N=56$. First we consider

$$
D_{1}(q)=\frac{\eta^{4}(2 z) \eta^{2}(4 z) \eta(14 z) \eta(56 z)}{\eta^{2}(z) \eta(8 z) \eta(28 z)} .
$$

Then we have 
Table 3.5.10(a)

\begin{tabular}{r|rrrrrrr}
\hline$\delta$ & 1 & 2 & 4 & 8 & 14 & 28 & 56 \\
\hline$r_{\delta}$ & -2 & 4 & 2 & -1 & 1 & -1 & 1 \\
\hline
\end{tabular}

It can be seen from Table 3.5.10(a) that conditions (L1) and (L2) are satisfied.

Table 3.5.10(b)

\begin{tabular}{r|rrrrrrrr}
\hline$d \mid 56$ & 1 & 2 & 4 & 7 & 8 & 14 & 28 & 56 \\
\hline$\sum_{1 \leq \delta \mid 56} \frac{\operatorname{gcd}(d, \delta)^{2} \cdot r_{\delta}}{\delta}$ & $3 / 7$ & $54 / 7$ & 12 & 3 & $48 / 7$ & 18 & 12 & 48 \\
\hline
\end{tabular}

From Table 3.5.10(b) the condition $(L 4)$ is also satisfied. Thus $D_{1}(q) \in S_{2}\left(\Gamma_{0}(56), \chi_{6}\right)$. Secondly we consider

$$
D_{2}(q)=\frac{\eta^{2}(2 z) \eta^{4}(4 z) \eta(7 z) \eta(28 z)}{\eta(z) \eta^{2}(8 z) \eta(14 z)}
$$

Then we have

Table 3.5.11(a)

\begin{tabular}{r|rrrrrrr}
\hline$\delta$ & 1 & 2 & 4 & 7 & 8 & 14 & 28 \\
\hline$r_{\delta}$ & -1 & 2 & 4 & 1 & -2 & -1 & 1 \\
\hline
\end{tabular}

It can be seen from Table 3.5.11(a) that conditions (L1) and (L2) are satisfied.

Table 3.5.11(b)

\begin{tabular}{r|rrrrrrrr}
\hline$d \mid 56$ & 1 & 2 & 4 & 7 & 8 & 14 & 28 & 56 \\
\hline$\sum_{1 \leq \delta \mid 56} \frac{\operatorname{gcd}(d, \delta)^{2} \cdot r_{\delta}}{\delta}$ & $6 / 7$ & 6 & $108 / 7$ & 6 & $24 / 7$ & 6 & 36 & 24 \\
\hline
\end{tabular}

From Table 3.5.11(b) the condition $(L 4)$ is also satisfied. Thus $D_{2}(q) \in S_{2}\left(\Gamma_{0}(56), \chi_{6}\right)$.

Thirdly we consider

$$
D_{3}(q)=\frac{\eta^{3}(2 z) \eta(7 z) \eta^{2}(56 z)}{\eta(z) \eta(4 z)}
$$

Then we have 
Table 3.5.12(a)

\begin{tabular}{r|rrrrr}
\hline$\delta$ & 1 & 2 & 4 & 7 & 56 \\
\hline$r_{\delta}$ & -1 & 3 & -1 & 1 & 2 \\
\hline
\end{tabular}

It can be seen from Table 3.5.12(a) that conditions (L1) and (L2) are satisfied.

Table 3.5.12(b)

\begin{tabular}{r|rrrrrrrr}
\hline$d \mid 56$ & 1 & 2 & 4 & 7 & 8 & 14 & 28 & 56 \\
\hline$\sum_{1 \leq \delta \mid 56} \frac{\operatorname{gcd}(d, \delta)^{2} \cdot r_{\delta}}{\delta}$ & $3 / 7$ & $30 / 7$ & $12 / 7$ & 9 & $24 / 7$ & 18 & 36 & 120 \\
\hline
\end{tabular}

From Table 3.5.12(b) the condition $(L 4)$ is also satisfied. Thus $D_{3}(q) \in S_{2}\left(\Gamma_{0}(56), \chi_{6}\right)$.

Fourthly we consider

$$
D_{4}(q)=\frac{\eta^{3}(4 z) \eta^{2}(7 z) \eta(56 z)}{\eta(2 z) \eta(8 z)}
$$

Then we have

Table 3.5.13(a)

\begin{tabular}{r|rrrrr}
\hline$\delta$ & 2 & 4 & 7 & 8 & 56 \\
\hline$r_{\delta}$ & -1 & 3 & 2 & -1 & 1 \\
\hline
\end{tabular}

It can be seen from Table 3.5.13(a) that conditions (L1) and (L2) are satisfied.

Table 3.5.13(b)

\begin{tabular}{r|rrrrrrrr}
\hline$d \mid 56$ & 1 & 2 & 4 & 7 & 8 & 14 & 28 & 56 \\
\hline$\sum_{1 \leq \delta \mid 56} \frac{\operatorname{gcd}(d, \delta)^{2} \cdot r_{\delta}}{\delta}$ & $3 / 7$ & $6 / 7$ & $60 / 7$ & 15 & $24 / 7$ & 18 & 36 & 72 \\
\hline
\end{tabular}

From Table 3.5.13(b) the condition $(L 4)$ is also satisfied. Thus $D_{4}(q) \in S_{2}\left(\Gamma_{0}(56), \chi_{6}\right)$.

Fifthly we consider

$$
D_{5}(q)=\frac{\eta(2 z) \eta(8 z) \eta^{4}(14 z) \eta^{2}(28 z)}{\eta(4 z) \eta^{2}(7 z) \eta(56 z)}
$$

Then we have 
Table 3.5.14(a)

\begin{tabular}{r|rrrrrrr}
\hline$\delta$ & 2 & 4 & 7 & 8 & 14 & 28 & 56 \\
\hline$r_{\delta}$ & 1 & -1 & -2 & 1 & 4 & 2 & -1 \\
\hline
\end{tabular}

It can be seen from Table 3.5.14(a) that conditions $(L 1)$ and $(L 2)$ are satisfied.

Table 3.5.14(b)

\begin{tabular}{r|rrrrrrrr}
\hline$d \mid 56$ & 1 & 2 & 4 & 7 & 8 & 14 & 28 & 56 \\
\hline$\sum_{1 \leq \delta \mid 56} \frac{\operatorname{gcd}(d, \delta)^{2} \cdot r_{\delta}}{\delta}$ & $3 / 7$ & $18 / 7$ & $12 / 7$ & 3 & $48 / 7$ & 54 & 84 & 48 \\
\hline
\end{tabular}

From Table 3.5.14(b) the condition ( $L 4)$ is also satisfied. Thus $D_{5}(q) \in S_{2}\left(\Gamma_{0}(56), \chi_{6}\right)$. Sixthly we consider

$$
D_{6}(q)=\frac{\eta(z) \eta^{2}(8 z) \eta^{3}(14 z)}{\eta(7 z) \eta(28 z)}
$$

Then we have

Table 3.5.15(a)

\begin{tabular}{r|rrrrr}
\hline$\delta$ & 1 & 7 & 8 & 14 & 28 \\
\hline$r_{\delta}$ & 1 & -1 & 2 & 3 & -1 \\
\hline
\end{tabular}

It can be seen from Table 3.5.15(a) that conditions (L1) and (L2) are satisfied.

Table 3.5.15(b)

\begin{tabular}{r|rrrrrrrr}
\hline$d \mid 56$ & 1 & 2 & 4 & 7 & 8 & 14 & 28 & 56 \\
\hline$\sum_{1 \leq \delta \mid 56} \frac{\operatorname{gcd}(d, \delta)^{2} \cdot r_{\delta}}{\delta}$ & $9 / 7$ & $18 / 7$ & $36 / 7$ & 3 & $120 / 7$ & 30 & 12 & 24 \\
\hline
\end{tabular}

From Table 3.5.15(b) the condition $(L 4)$ is also satisfied. Thus $D_{6}(q) \in$ $S_{2}\left(\Gamma_{0}(56), \chi_{6}\right)$.

Theorem 3.5.3. (a) $\left\{D_{1}(q), \ldots, D_{6}(q)\right\}$ is a basis for $S_{2}\left(\Gamma_{0}(56), \chi_{6}\right)$.

(b) $\left\{E_{2, \chi_{4}, \chi_{1}}(q), E_{2, \chi_{1}, \chi_{4}}(q), E_{2, \chi_{6}, \chi_{0}}(q), E_{2, \chi_{0}, \chi_{6}}(q)\right\}$ is a basis for $E_{2}\left(\Gamma_{0}(56), \chi_{6}\right)$. 
(c) $\left\{E_{2, \chi_{4}, \chi_{1}}(q), E_{2, \chi_{1}, \chi_{4}}(q), E_{2, \chi_{6}, \chi_{0}}(q), E_{2, \chi_{0}, \chi_{6}}(q)\right\}$ together with $D_{k}(q)(1 \leq k \leq 6)$ constitute a basis for $M_{2}\left(\Gamma_{0}(56), \chi_{6}\right)$.

Proof. (a) By Theorem 3.5.2, $D_{k}(q)(1 \leq k \leq 6) \in S_{2}\left(\Gamma_{0}(56), \chi_{6}\right)$. There is no linear relationship among them. By Example 2.3.3, we have $\operatorname{dim} S_{2}\left(\Gamma_{0}(56), \chi_{6}\right)=6$. Thus $D_{k}(q)(1 \leq k \leq 6)$ constitute a basis for $S_{2}\left(\Gamma_{0}(56), \chi_{6}\right)$.

(b) By Example 2.3.3, we have $\operatorname{dim} E_{2}\left(\Gamma_{0}(56), \chi_{6}\right)=4$. By Theorem 2.2.3, $\left\{E_{2, \chi_{4}, \chi_{1}}(q)\right.$, $\left.E_{2, \chi_{1}, \chi_{4}}(q), E_{2, \chi_{6}, \chi_{0}}(q), E_{2, \chi_{0}, \chi_{6}}(q)\right\}$ constitute a basis for $E_{2}\left(\Gamma_{0}(56), \chi_{6}\right)$.

(c) By Example 2.3.3, we have $\operatorname{dim} M_{2}\left(\Gamma_{0}(56), \chi_{6}\right)=10$. Therefore, by (2.1.1) $\left\{E_{2, \chi_{4}, \chi_{1}}(q), E_{2, \chi_{1}, \chi_{4}}(q), E_{2, \chi_{6}, \chi_{0}}(q), E_{2, \chi_{0}, \chi_{6}}(q)\right\}$ together with $D_{k}(q)(1 \leq k \leq 6)$ constitute a basis for $M_{2}\left(\Gamma_{0}(56), \chi_{6}\right)$.

Theorem 3.5.4. Let $\chi_{0}$ be the trivial character and $\chi_{1}, \chi_{4}, \chi_{6}$ be as in (3.1.3) and (3.1.4). Then

$$
\begin{aligned}
\text { (a) } \varphi^{2}(q) \varphi\left(q^{2}\right) \varphi\left(q^{7}\right)= & -\frac{2}{5} E_{2, \chi_{4}, \chi_{1}}(q)+\frac{7}{10} E_{2, \chi_{1}, \chi_{4}}(q)+\frac{14}{5} E_{2, \chi_{6}, \chi_{0}}(q)-\frac{1}{10} E_{2, \chi_{0}, \chi_{6}}(q) \\
& -\frac{1}{10} D_{1}(q)+\frac{6}{5} D_{2}(q)-\frac{7}{5} D_{3}(q)-\frac{7}{10} D_{5}(q)-\frac{1}{5} D_{6}(q), \\
\text { (b) } \varphi^{3}\left(q^{2}\right) \varphi\left(q^{7}\right)=- & \frac{1}{5} E_{2, \chi_{4}, \chi_{1}}(q)+\frac{7}{10} E_{2, \chi_{1}, \chi_{4}}(q)+\frac{7}{5} E_{2, \chi_{6}, \chi_{0}}(q)-\frac{1}{10} E_{2, \chi_{0}, \chi_{6}}(q) \\
& +\frac{51}{20} D_{1}(q)+\frac{9}{10} D_{2}(q)+\frac{63}{10} D_{3}(q)-\frac{63}{5} D_{4}(q)-\frac{63}{20} D_{5}(q)-\frac{27}{10} D_{6}(q), \\
& -\frac{3}{2} D_{1}(q)+\frac{3}{5} D_{3}(q)+\frac{12}{5} D_{4}(q)+\frac{3}{2} D_{5}(q)-\frac{3}{5} D_{6}(q), \\
\text { (c) } \varphi\left(q^{2}\right) \varphi^{3}\left(q^{7}\right)= & \frac{2}{5} E_{2, \chi_{4}, \chi_{1}}(q)-\frac{1}{10} E_{2, \chi_{1}, \chi_{4}}(q)+\frac{2}{5} E_{2, \chi_{6}, \chi_{0}}(q)-\frac{1}{10} E_{2, \chi_{0}, \chi_{6}}(q) \\
\text { (d) } \varphi^{3}(q) \varphi\left(q^{14}\right)= & -\frac{2}{5} E_{2, \chi_{4}, \chi_{1}}(q)+\frac{7}{10} E_{2, \chi_{1}, \chi_{4}}(q)+\frac{14}{5} E_{2, \chi_{6}, \chi_{0}}(q)-\frac{1}{10} E_{2, \chi_{0}, \chi_{6}}(q) \\
& +\frac{9}{10} D_{1}(q)+\frac{6}{5} D_{2}(q)+\frac{63}{5} D_{3}(q)+\frac{63}{10} D_{5}(q)+\frac{9}{5} D_{6}(q),
\end{aligned}
$$


(e) $\varphi(q) \varphi^{2}\left(q^{2}\right) \varphi\left(q^{14}\right)=-\frac{1}{5} E_{2, \chi_{4}, \chi_{1}}(q)+\frac{7}{10} E_{2, \chi_{1}, \chi_{4}}(q)+\frac{7}{5} E_{2, \chi_{6}, \chi_{0}}(q)-\frac{1}{10} E_{2, \chi_{0}, \chi_{6}}(q)$

$+\frac{21}{20} D_{1}(q)-\frac{1}{10} D_{2}(q)-\frac{7}{10} D_{3}(q)+\frac{7}{5} D_{4}(q)+\frac{7}{20} D_{5}(q)$

$+\frac{3}{10} D_{6}(q)$

(f) $\varphi(q) \varphi^{2}\left(q^{7}\right) \varphi\left(q^{14}\right)=\frac{2}{5} E_{2, \chi_{4}, \chi_{1}}(q)-\frac{1}{10} E_{2, \chi_{1}, \chi_{4}}(q)+\frac{2}{5} E_{2, \chi_{6}, \chi_{0}}(q)-\frac{1}{10} E_{2, \chi_{0}, \chi_{6}}(q)$

$-\frac{1}{2} D_{1}(q)-\frac{7}{5} D_{3}(q)+\frac{12}{5} D_{4}(q)+\frac{1}{2} D_{5}(q)+\frac{7}{5} D_{6}(q)$

(g) $\varphi\left(q^{2}\right) \varphi\left(q^{7}\right) \varphi^{2}\left(q^{14}\right)=\frac{1}{5} E_{2, \chi_{4}, \chi_{1}}(q)-\frac{1}{10} E_{2, \chi_{1}, \chi_{4}}(q)+\frac{1}{5} E_{2, \chi_{6}, \chi_{0}}(q)-\frac{1}{10} E_{2, \chi_{0}, \chi_{6}}(q)$

$+\frac{1}{20} D_{1}(q)-\frac{1}{10} D_{2}(q)-\frac{3}{10} D_{3}(q)-\frac{1}{5} D_{4}(q)+\frac{27}{20} D_{5}(q)$ $-\frac{1}{10} D_{6}(q)$

(h) $\varphi(q) \varphi^{3}\left(q^{14}\right)=\frac{1}{5} E_{2, \chi_{4}, \chi_{1}}(q)-\frac{1}{10} E_{2, \chi_{1}, \chi_{4}}(q)+\frac{1}{5} E_{2, \chi_{6}, \chi_{0}}(q)-\frac{1}{10} E_{2, \chi_{0}, \chi_{6}}(q)$

$$
\begin{aligned}
& -\frac{9}{20} D_{1}(q)+\frac{9}{10} D_{2}(q)+\frac{27}{10} D_{3}(q)+\frac{9}{5} D_{4}(q)-\frac{3}{20} D_{5}(q) \\
& +\frac{9}{10} D_{6}(q) .
\end{aligned}
$$

Proof. Let $\left(a_{1}, a_{2}, a_{3}, a_{4}\right)$ be one of the quadratic forms listed in the fourth column of Table 3.1.1. By Theorem 3.5.1, $\varphi\left(q^{a_{1}}\right) \varphi\left(q^{a_{2}}\right) \varphi\left(q^{a_{3}}\right) \varphi\left(q^{a_{4}}\right) \in M_{2}\left(\Gamma_{0}(56), \chi_{6}\right)$. Therefore, by Theorem 3.5.3(c), $\varphi\left(q^{a_{1}}\right) \varphi\left(q^{a_{2}}\right) \varphi\left(q^{a_{3}}\right) \varphi\left(q^{a_{4}}\right)$ must be a linear combination of $E_{2, \chi_{1}, \chi_{4}}(q), E_{2, \chi_{4}, \chi_{1}}(q), E_{2, \chi_{6}, \chi_{0}}(q), E_{2, \chi_{0}, \chi_{6}}(q)$ and $D_{k}(q)(1 \leq k \leq 6)$, namely

$$
\begin{aligned}
\varphi\left(q^{a_{1}}\right) \varphi\left(q^{a_{2}}\right) \varphi\left(q^{a_{3}}\right) \varphi\left(q^{a_{4}}\right)= & x_{1} E_{2, \chi_{4}, \chi_{1}}(q)+x_{2} E_{2, \chi_{1}, \chi_{4}}(q)+x_{3} E_{2, \chi_{6}, \chi_{0}}(q)+x_{4} E_{2, \chi_{0}, \chi_{6}}(q) \\
& +y_{1} D_{1}(q)+y_{2} D_{2}(q)+y_{3} D_{3}(q)+y_{4} D_{4}(q)+y_{5} D_{5}(q) \\
& +y_{6} D_{6}(q) .
\end{aligned}
$$

We equate the first fourteen coefficients of $q^{n}$ on both sides of the equation above to obtain a system of linear equations with the unknowns $x_{1}, x_{2}, x_{3}, x_{4}, y_{1}, y_{2}, y_{3}, y_{4}, y_{5}, y_{6}$. Then, using MAPLE we solve the system to find the asserted coefficients. 
Theorem 3.5.5. Let $n \in \mathbb{N}$. Let $\sigma_{\chi_{i}, \chi_{j}}(n)$ be as in (2.2.1) for $i, j \in\{0,1,4,6\}$. Then

(a) $N(1,1,2,7 ; n)=-\frac{2}{5} \sigma_{\chi_{4}, \chi_{1}}(n)+\frac{7}{10} \sigma_{\chi_{1}, \chi_{4}}(n)+\frac{14}{5} \sigma_{\chi_{6}, \chi_{0}}(n)-\frac{1}{10} \sigma_{\chi_{0}, \chi_{6}}(n)$

$$
-\frac{1}{10} d_{1}(n)+\frac{6}{5} d_{2}(n)-\frac{7}{5} d_{3}(n)-\frac{7}{10} d_{5}(n)-\frac{1}{5} d_{6}(n)
$$

(b) $N(2,2,2,7 ; n)=-\frac{1}{5} \sigma_{\chi_{4}, \chi_{1}}(n)+\frac{7}{10} \sigma_{\chi_{1}, \chi_{4}}(n)+\frac{7}{5} \sigma_{\chi_{6}, \chi_{0}}(n)-\frac{1}{10} \sigma_{\chi_{0}, \chi_{6}}(n)$

$$
\begin{aligned}
& +\frac{51}{20} d_{1}(n)+\frac{9}{10} d_{2}(n)+\frac{63}{10} d_{3}(n)-\frac{63}{5} d_{4}(n)-\frac{63}{20} d_{5}(n) \\
& -\frac{27}{10} d_{6}(n),
\end{aligned}
$$

(c) $N(2,7,7,7 ; n)=\frac{2}{5} \sigma_{\chi_{4}, \chi_{1}}(n)-\frac{1}{10} \sigma_{\chi_{1}, \chi_{4}}(n)+\frac{2}{5} \sigma_{\chi_{6}, \chi_{0}}(n)-\frac{1}{10} \sigma_{\chi_{0}, \chi_{6}}(n)$

$$
-\frac{3}{2} d_{1}(n)+\frac{3}{5} d_{3}(n)+\frac{12}{5} d_{4}(n)+\frac{3}{2} d_{5}(n)-\frac{3}{5} d_{6}(n),
$$

(d) $N(1,1,1,14 ; n)=-\frac{2}{5} \sigma_{\chi_{4}, \chi_{1}}(n)+\frac{7}{10} \sigma_{\chi_{1}, \chi_{4}}(n)+\frac{14}{5} \sigma_{\chi_{6}, \chi_{0}}(n)-\frac{1}{10} \sigma_{\chi_{0}, \chi_{6}}(n)$

$$
+\frac{9}{10} d_{1}(n)+\frac{6}{5} d_{2}(n)+\frac{63}{5} d_{3}(n)+\frac{63}{10} d_{5}(n)+\frac{9}{5} d_{6}(n),
$$

(e) $N(1,2,2,14 ; n)=-\frac{1}{5} \sigma_{\chi_{4}, \chi_{1}}(n)+\frac{7}{10} \sigma_{\chi_{1}, \chi_{4}}(n)+\frac{7}{5} \sigma_{\chi_{6}, \chi_{0}}(n)-\frac{1}{10} \sigma_{\chi_{0}, \chi_{6}}(n)$

$$
\begin{aligned}
+ & \frac{21}{20} d_{1}(n)-\frac{1}{10} d_{2}(n)-\frac{7}{10} d_{3}(n)+\frac{7}{5} d_{4}(n)+\frac{7}{20} d_{5}(n) \\
& +\frac{3}{10} d_{6}(n),
\end{aligned}
$$

(f) $N(1,7,7,14 ; n)=\frac{2}{5} \sigma_{\chi_{4}, \chi_{1}}(n)-\frac{1}{10} \sigma_{\chi_{1}, \chi_{4}}(n)+\frac{2}{5} \sigma_{\chi_{6}, \chi_{0}}(n)-\frac{1}{10} \sigma_{\chi_{0}, \chi_{6}}(n)$

$$
-\frac{1}{2} d_{1}(n)-\frac{7}{5} d_{3}(n)+\frac{12}{5} d_{4}(n)+\frac{1}{2} d_{5}(n)+\frac{7}{5} d_{6}(n),
$$

(g) $N(2,7,14,14 ; n)=\frac{1}{5} \sigma_{\chi_{4}, \chi_{1}}(n)-\frac{1}{10} \sigma_{\chi_{1}, \chi_{4}}(n)+\frac{1}{5} \sigma_{\chi_{6}, \chi_{0}}(n)-\frac{1}{10} \sigma_{\chi_{0}, \chi_{6}}(n)$

$$
\begin{aligned}
+ & \frac{1}{20} d_{1}(n)-\frac{1}{10} d_{2}(n)-\frac{3}{10} d_{3}(n)-\frac{1}{5} d_{4}(n)+\frac{27}{20} d_{5}(n) \\
& -\frac{1}{10} d_{6}(n),
\end{aligned}
$$


(h) $N(1,14,14,14 ; n)=\frac{1}{5} \sigma_{\chi_{4}, \chi_{1}}(n)-\frac{1}{10} \sigma_{\chi_{1}, \chi_{4}}(n)+\frac{1}{5} \sigma_{\chi_{6}, \chi_{0}}(n)-\frac{1}{10} \sigma_{\chi_{0}, \chi_{6}}(n)$

$$
\begin{aligned}
& -\frac{9}{20} d_{1}(n)+\frac{9}{10} d_{2}(n)+\frac{27}{10} d_{3}(n)+\frac{9}{5} d_{4}(n)-\frac{3}{20} d_{5}(n) \\
& +\frac{9}{10} d_{6}(n) .
\end{aligned}
$$

Proof. Appealing to (2.1.6), (3.5.1)-(3.5.4) and Theorem 3.5.4, we have

(a) $\sum_{n=0}^{\infty} N(1,1,2,7 ; n) q^{n}=\varphi^{2}(q) \varphi\left(q^{2}\right) \varphi\left(q^{7}\right)$

$$
\begin{aligned}
= & -\frac{2}{5} E_{2, \chi_{4}, \chi_{1}}(q)+\frac{7}{10} E_{2, \chi_{1}, \chi_{4}}(q)+\frac{14}{5} E_{2, \chi_{6}, \chi_{0}}(q)-\frac{1}{10} E_{2, \chi_{0}, \chi_{6}}(q) \\
& -\frac{1}{10} D_{1}(q)+\frac{6}{5} D_{2}(q)-\frac{7}{5} D_{3}(q)-\frac{7}{10} D_{5}(q)-\frac{1}{5} D_{6}(q), \\
= & 1+\sum_{n=1}^{\infty}\left(-\frac{2}{5} \sigma_{\chi_{4}, \chi_{1}}(n)+\frac{7}{10} \sigma_{\chi_{1}, \chi_{4}}(n)+\frac{14}{5} \sigma_{\chi_{6}, \chi_{0}}(n)-\frac{1}{10} \sigma_{\chi_{0}, \chi_{6}}(n)\right. \\
& \left.-\frac{1}{10} d_{1}(n)+\frac{6}{5} d_{2}(n)-\frac{7}{5} d_{3}(n)-\frac{7}{10} d_{5}(n)-\frac{1}{5} d_{6}(n)\right) q^{n},
\end{aligned}
$$

(b) $\sum_{n=0}^{\infty} N(2,2,2,7 ; n) q^{n}=\varphi^{3}\left(q^{2}\right) \varphi\left(q^{7}\right)$

$$
\begin{aligned}
= & -\frac{1}{5} E_{2, \chi_{4}, \chi_{1}}(q)+\frac{7}{10} E_{2, \chi_{1}, \chi_{4}}(q)+\frac{7}{5} E_{2, \chi_{6}, \chi_{0}}(q)-\frac{1}{10} E_{2, \chi_{0}, \chi_{6}}(q)+\frac{51}{20} D_{1}(q) \\
& +\frac{9}{10} D_{2}(q)+\frac{63}{10} D_{3}(q)-\frac{63}{5} D_{4}(q)-\frac{63}{20} D_{5}(q)-\frac{27}{10} D_{6}(q), \\
= & 1+\sum_{n=1}^{\infty}\left(-\frac{1}{5} \sigma_{\chi_{4}, \chi_{1}}(n)+\frac{7}{10} \sigma_{\chi_{1}, \chi_{4}}(n)+\frac{7}{5} \sigma_{\chi_{6}, \chi_{0}}(n)-\frac{1}{10} \sigma_{\chi_{0}, \chi_{6}}(n)+\frac{51}{20} d_{1}(n)\right. \\
& \left.+\frac{9}{10} d_{2}(n)+\frac{63}{10} d_{3}(n)-\frac{63}{5} d_{4}(n)-\frac{63}{20} d_{5}(n)-\frac{27}{10} d_{6}(n)\right) q^{n},
\end{aligned}
$$


(c) $\sum_{n=0}^{\infty} N(2,7,7,7 ; n) q^{n}=\varphi\left(q^{2}\right) \varphi^{3}\left(q^{7}\right)$

$=\frac{2}{5} E_{2, \chi_{4}, \chi_{1}}(q)-\frac{1}{10} E_{2, \chi_{1}, \chi_{4}}(q)+\frac{2}{5} E_{2, \chi_{6}, \chi_{0}}(q)-\frac{1}{10} E_{2, \chi_{0}, \chi_{6}}(q)-\frac{3}{2} D_{1}(q)$

$+\frac{3}{5} D_{3}(q)+\frac{12}{5} D_{4}(q)+\frac{3}{2} D_{5}(q)-\frac{3}{5} D_{6}(q)$,

$=1+\sum_{n=1}^{\infty}\left(\frac{2}{5} \sigma_{\chi_{4}, \chi_{1}}(n)-\frac{1}{10} \sigma_{\chi_{1}, \chi_{4}}(n)+\frac{2}{5} \sigma_{\chi_{6}, \chi_{0}}(n)-\frac{1}{10} \sigma_{\chi_{0}, \chi_{6}}(n)\right.$

$\left.-\frac{3}{2} d_{1}(n)+\frac{3}{5} d_{3}(n)+\frac{12}{5} d_{4}(n)+\frac{3}{2} d_{5}(n)-\frac{3}{5} d_{6}(n)\right) q^{n}$,

(d) $\sum_{n=0}^{\infty} N(1,1,1,14 ; n) q^{n}=\varphi^{3}(q) \varphi\left(q^{14}\right)$

$=-\frac{2}{5} E_{2, \chi_{4}, \chi_{1}}(q)+\frac{7}{10} E_{2, \chi_{1}, \chi_{4}}(q)+\frac{14}{5} E_{2, \chi_{6}, \chi_{0}}(q)-\frac{1}{10} E_{2, \chi_{0}, \chi_{6}}(q)+\frac{9}{10} D_{1}(q)$

$+\frac{6}{5} D_{2}(q)+\frac{63}{5} D_{3}(q)+\frac{63}{10} D_{5}(q)+\frac{9}{5} D_{6}(q)$,

$=1+\sum_{n=1}^{\infty}\left(-\frac{2}{5} \sigma_{\chi_{4}, \chi_{1}}(n)+\frac{7}{10} \sigma_{\chi_{1}, \chi_{4}}(n)+\frac{14}{5} \sigma_{\chi_{6}, \chi_{0}}(n)-\frac{1}{10} \sigma_{\chi_{0}, \chi_{6}}(n)+\frac{9}{10} d_{1}(n)\right.$

$\left.+\frac{6}{5} d_{2}(n)+\frac{63}{5} d_{3}(n)+\frac{63}{10} d_{5}(n)+\frac{9}{5} d_{6}(n)\right) q^{n}$

(e) $\sum_{n=0}^{\infty} N(1,2,2,14 ; n) q^{n}=\varphi(q) \varphi^{2}\left(q^{2}\right) \varphi\left(q^{14}\right)$

$=-\frac{1}{5} E_{2, \chi_{4}, \chi_{1}}(q)+\frac{7}{10} E_{2, \chi_{1}, \chi_{4}}(q)+\frac{7}{5} E_{2, \chi_{6}, \chi_{0}}(q)-\frac{1}{10} E_{2, \chi_{0}, \chi_{6}}(q)$

$+\frac{21}{20} D_{1}(q)-\frac{1}{10} D_{2}(q)-\frac{7}{10} D_{3}(q)+\frac{7}{5} D_{4}(q)+\frac{7}{20} D_{5}(q)+\frac{3}{10} D_{6}(q)$,

$=1+\sum_{n=1}^{\infty}\left(-\frac{1}{5} \sigma_{\chi_{4}, \chi_{1}}(n)+\frac{7}{10} \sigma_{\chi_{1}, \chi_{4}}(n)+\frac{7}{5} \sigma_{\chi_{6}, \chi_{0}}(n)-\frac{1}{10} \sigma_{\chi_{0}, \chi_{6}}(n)\right.$

$\left.+\frac{21}{20} d_{1}(n)-\frac{1}{10} d_{2}(n)-\frac{7}{10} d_{3}(n)+\frac{7}{5} d_{4}(n)+\frac{7}{20} d_{5}(n)+\frac{3}{10} d_{6}(n)\right) q^{n}$, 
(f)

$$
\begin{aligned}
\sum_{n=0}^{\infty} N(1,7,7,14 ; n) q^{n}=\varphi(q) \varphi^{2}\left(q^{7}\right) \varphi\left(q^{14}\right) \\
=\frac{2}{5} E_{2, \chi_{4}, \chi_{1}}(q)-\frac{1}{10} E_{2, \chi_{1}, \chi_{4}}(q)+\frac{2}{5} E_{2, \chi_{6}, \chi_{0}}(q)-\frac{1}{10} E_{2, \chi_{0}, \chi_{6}}(q)-\frac{1}{2} D_{1}(q) \\
\quad-\frac{7}{5} D_{3}(q)+\frac{12}{5} D_{4}(q)+\frac{1}{2} D_{5}(q)+\frac{7}{5} D_{6}(q), \\
=1+\sum_{n=1}^{\infty}\left(\frac{2}{5} \sigma_{\chi_{4}, \chi_{1}}(n)-\frac{1}{10} \sigma_{\chi_{1}, \chi_{4}}(n)+\frac{2}{5} \sigma_{\chi_{6}, \chi_{0}}(n)-\frac{1}{10} \sigma_{\chi_{0}, \chi_{6}}(n)-\frac{1}{2} d_{1}(n)\right. \\
\left.\quad-\frac{7}{5} d_{3}(n)+\frac{12}{5} d_{4}(n)+\frac{1}{2} d_{5}(n)+\frac{7}{5} d_{6}(n)\right) q^{n}
\end{aligned}
$$

(g) $\sum_{n=0}^{\infty} N(2,7,14,14 ; n) q^{n}=\varphi\left(q^{2}\right) \varphi\left(q^{7}\right) \varphi^{2}\left(q^{14}\right)$

$$
\begin{aligned}
= & \frac{1}{5} E_{2, \chi_{4}, \chi_{1}}(q)-\frac{1}{10} E_{2, \chi_{1}, \chi_{4}}(q)+\frac{1}{5} E_{2, \chi_{6}, \chi_{0}}(q)-\frac{1}{10} E_{2, \chi_{0}, \chi_{6}}(q) \\
& +\frac{1}{20} D_{1}(q)-\frac{1}{10} D_{2}(q)-\frac{3}{10} D_{3}(q)-\frac{1}{5} D_{4}(q)+\frac{27}{20} D_{5}(q)-\frac{1}{10} D_{6}(q), \\
= & 1+\sum_{n=1}^{\infty}\left(\frac{1}{5} \sigma_{\chi_{4}, \chi_{1}}(n)-\frac{1}{10} \sigma_{\chi_{1}, \chi_{4}}(n)+\frac{1}{5} \sigma_{\chi_{6}, \chi_{0}}(n)-\frac{1}{10} \sigma_{\chi_{0}, \chi_{6}}(n)\right. \\
& \left.+\frac{1}{20} d_{1}(n)-\frac{1}{10} d_{2}(n)-\frac{3}{10} d_{3}(n)-\frac{1}{5} d_{4}(n)+\frac{27}{20} d_{5}(n)-\frac{1}{10} d_{6}(n)\right) q^{n}
\end{aligned}
$$

(h) $\sum_{n=0}^{\infty} N(1,14,14,14 ; n) q^{n}=\varphi(q) \varphi^{3}\left(q^{14}\right)$

$$
\begin{aligned}
= & \frac{1}{5} E_{2, \chi_{4}, \chi_{1}}(q)-\frac{1}{10} E_{2, \chi_{1}, \chi_{4}}(q)+\frac{1}{5} E_{2, \chi_{6}, \chi_{0}}(q)-\frac{1}{10} E_{2, \chi_{0}, \chi_{6}}(q)-\frac{9}{20} D_{1}(q) \\
& +\frac{9}{10} D_{2}(q)+\frac{27}{10} D_{3}(q)+\frac{9}{5} D_{4}(q)-\frac{3}{20} D_{5}(q)+\frac{9}{10} D_{6}(q), \\
= & 1+\sum_{n=1}^{\infty}\left(\frac{1}{5} \sigma_{\chi_{4}, \chi_{1}}(n)-\frac{1}{10} \sigma_{\chi_{1}, \chi_{4}}(n)+\frac{1}{5} \sigma_{\chi_{6}, \chi_{0}}(n)-\frac{1}{10} \sigma_{\chi_{0}, \chi_{6}}(n)-\frac{9}{20} d_{1}(n)\right. \\
& \left.+\frac{9}{10} d_{2}(n)+\frac{27}{10} d_{3}(n)+\frac{9}{5} d_{4}(n)-\frac{3}{20} d_{5}(n)+\frac{9}{10} d_{6}(n)\right) q^{n} .
\end{aligned}
$$

Equating the coefficients of $q^{n}$ on both sides of equations (a)-(h) yields the results.

The values of $N\left(a_{1}, a_{2}, a_{3}, a_{4} ; n\right)$ for $1 \leq n \leq 20$ for the quadratic forms $\left(a_{1}, a_{2}, a_{3}, a_{4}\right)$ in Theorem 3.5.5 are given in Table 3.5.16. 
Table 3.5.16

\begin{tabular}{l|rrrrrrrrrrrrrrrrrrrr}
\hline \multicolumn{1}{c|}{$n$} & 1 & 2 & 3 & 4 & 5 & 6 & 7 & 8 & 9 & 10 & 11 & 12 & 13 & 14 & 15 & 16 & 17 & 18 & 19 & 20 \\
\hline$N(1,1,2,7 ; n)$ & 4 & 6 & 8 & 12 & 8 & 8 & 18 & 14 & 24 & 40 & 32 & 40 & 40 & 32 & 28 & 36 & 64 & 46 & 72 & 72 \\
$N(2,2,2,7 ; n)$ & 0 & 6 & 0 & 12 & 0 & 8 & 2 & 6 & 12 & 24 & 24 & 24 & 16 & 0 & 12 & 12 & 48 & 30 & 48 & 24 \\
$N(2,7,7,7 ; n)$ & 0 & 2 & 0 & 0 & 0 & 0 & 6 & 2 & 12 & 0 & 0 & 0 & 0 & 12 & 12 & 24 & 0 & 2 & 0 & 0 \\
$N(1,1,1,14 ; n)$ & 6 & 12 & 8 & 6 & 24 & 24 & 0 & 12 & 30 & 24 & 24 & 8 & 24 & 50 & 12 & 30 & 64 & 48 & 72 & 72 \\
$N(1,2,2,14 ; n)$ & 2 & 4 & 8 & 6 & 8 & 8 & 0 & 12 & 10 & 8 & 24 & 8 & 8 & 18 & 4 & 14 & 32 & 24 & 40 & 40 \\
$N(1,7,7,14 ; n)$ & 2 & 0 & 0 & 2 & 0 & 0 & 4 & 8 & 2 & 0 & 8 & 0 & 0 & 6 & 12 & 10 & 0 & 12 & 0 & 0 \\
$N(2,7,14,14 ; n)$ & 0 & 2 & 0 & 0 & 0 & 0 & 2 & 2 & 4 & 0 & 0 & 0 & 0 & 4 & 4 & 8 & 0 & 2 & 0 & 0 \\
$N(1,14,14,14 ; n)$ & 2 & 0 & 0 & 2 & 0 & 0 & 0 & 0 & 2 & 0 & 0 & 0 & 0 & 6 & 12 & 2 & 0 & 12 & 0 & 0 \\
\hline
\end{tabular}

Again one can verify the values in Table 3.5.16 by using (2.2.1), (3.1.3), (3.1.4) and

Table 3.5.1. 


\section{Chapter 4}

\section{Representations by Octonary}

\section{Quadratic Forms and Future Work}

In this chapter we extend our work to determine explicit formulae for the number of representations of $n$ by the octonary quadratic forms $x_{1}^{2}+x_{2}^{2}+x_{3}^{2}+x_{4}^{2}+7\left(x_{5}^{2}+x_{6}^{2}+x_{7}^{2}+\right.$ $\left.x_{8}^{2}\right), x_{1}^{2}+x_{2}^{2}+7\left(x_{3}^{2}+x_{4}^{2}+x_{5}^{2}+x_{6}^{2}+x_{7}^{2}+x_{8}^{2}\right)$ and $x_{1}^{2}+x_{2}^{2}+x_{3}^{2}+x_{4}^{2}+x_{5}^{2}+x_{6}^{2}+7\left(x_{7}^{2}+x_{8}^{2}\right)$, which we denote by $N\left(1^{4}, 7^{4} ; n\right), N\left(1^{2}, 7^{6} ; n\right)$ and $N\left(1^{6}, 7^{2} ; n\right)$ respectively. A formula for $N\left(1^{8} ; n\right)$ is given in $[2]$ and $[30]$.

\subsection{Representations by Octonary Quadratic Forms}

\section{with Coefficients 1 and 7}

For $a_{1}, \ldots, a_{8} \in \mathbb{N}$ and $n \in \mathbb{N}_{0}$, we define

$$
N\left(a_{1}, \ldots, a_{8} ; n\right):=\operatorname{card}\left\{\left(x_{1}, \ldots, x_{8}\right) \in \mathbb{Z}^{8} \mid n=a_{1} x_{1}^{2}+\cdots+a_{8} x_{8}^{2}\right\}
$$


For $i, j \in \mathbb{N}_{0}$ with $i+j=8$ we define

$$
N\left(1^{i}, 7^{j} ; n\right):=N(\underbrace{1, \ldots, 1}_{i}, \underbrace{7, \ldots, 7}_{j} ; n) .
$$

We give formulae for $N\left(1^{i}, 7^{j} ; n\right)$ in the cases $(i, j)=(4,4),(6,2)$ and $(2,6)$ in terms of $\sigma_{3}(n), \sigma_{3}(n / 2), \sigma_{3}(n / 4), \sigma_{3}(n / 7), \sigma_{3}(n / 14), \sigma_{3}(n / 28)$ and $v_{k}(n)(1 \leq k \leq 9)$ defined by

$$
\begin{aligned}
& V_{1}(q)=\sum_{n=1}^{\infty} v_{1}(n) q^{n}=\eta^{2}(z) \eta^{2}(2 z) \eta^{2}(7 z) \eta^{2}(14 z), \\
& V_{2}(q)=\sum_{n=1}^{\infty} v_{2}(n) q^{n}=\eta^{2}(2 z) \eta^{2}(4 z) \eta^{2}(14 z) \eta^{2}(28 z), \\
& V_{3}(q)=\sum_{n=1}^{\infty} v_{3}(n) q^{n}=\eta^{2}(z) \eta^{2}(7 z) \eta^{4}(14 z), \\
& V_{4}(q)=\sum_{n=1}^{\infty} v_{4}(n) q^{n}=\eta^{4}(2 z) \eta^{2}(4 z) \eta^{2}(28 z), \\
& V_{5}(q)=\sum_{n=1}^{\infty} v_{5}(n) q^{n}=\eta^{2}(z) \eta^{4}(2 z) \eta^{2}(7 z), \\
& V_{6}(q)=\sum_{n=1}^{\infty} v_{6}(n) q^{n}=\eta^{2}(4 z) \eta^{4}(14 z) \eta^{2}(28 z), \\
& V_{7}(q)=\sum_{n=1}^{\infty} v_{7}(n) q^{n}=\eta(z) \eta(4 z) \eta(7 z) \eta^{4}(14 z) \eta(28 z), \\
& V_{8}(q)=\sum_{n=1}^{\infty} v_{8}(n) q^{n}=\eta(z) \eta^{4}(2 z) \eta(4 z) \eta(7 z) \eta(28 z), \\
& V_{9}(q)=\sum_{n=1}^{\infty} v_{9}(n) q^{n}=\frac{\eta(z) \eta(2 z) \eta^{9}(7 z)}{\eta^{3}(14 z)} .
\end{aligned}
$$

Note that

$$
V_{2}(q)=V_{1}\left(q^{2}\right)
$$


so that

$$
v_{1}(2 n)=v_{2}(n)
$$

There is no linear relationship among the $V_{k}(q), 1 \leq k \leq 9$. The first twenty-eight values of $v_{k}(n)$, are given in Table 4.4.1.

Table 4.4.1

\begin{tabular}{|c|c|c|c|c|c|c|c|c|c|}
\hline$n$ & $v_{1}(n)$ & $v_{2}(n)$ & $v_{3}(n)$ & $v_{4}(n)$ & $v_{5}(n)$ & $v_{6}(n)$ & $v_{7}(n)$ & $v_{8}(n)$ & $v_{9}(n)$ \\
\hline 1 & 0 & 0 & 0 & 0 & 1 & 0 & 0 & 0 & 1 \\
\hline 2 & 1 & 0 & 0 & 0 & -2 & 0 & 0 & 1 & -1 \\
\hline 3 & -2 & 0 & 1 & 1 & -5 & 0 & 0 & -1 & -2 \\
\hline 4 & -3 & 1 & -2 & 0 & 10 & 0 & 1 & -5 & 1 \\
\hline 5 & 6 & 0 & -1 & -4 & 7 & 1 & -1 & 4 & 0 \\
\hline 6 & 2 & -2 & 2 & 0 & -10 & 0 & -1 & 5 & 2 \\
\hline 7 & 0 & 0 & 1 & 0 & 0 & 0 & 0 & 0 & 1 \\
\hline 8 & -1 & -3 & 2 & 0 & -22 & 0 & -1 & 11 & -9 \\
\hline 9 & -12 & 0 & -2 & 16 & -1 & -2 & 2 & -16 & 9 \\
\hline 10 & 4 & 6 & -2 & 0 & 38 & 0 & 1 & -19 & 16 \\
\hline 11 & 4 & 0 & 2 & -10 & -6 & 0 & 0 & 10 & -8 \\
\hline 12 & -2 & 2 & 0 & 0 & 8 & 0 & 0 & -4 & -2 \\
\hline 13 & 2 & 0 & -3 & -16 & -15 & -1 & 1 & 16 & -20 \\
\hline 14 & -7 & 0 & -2 & 0 & 0 & 0 & 1 & 0 & -9 \\
\hline 15 & 16 & 0 & -4 & 0 & 52 & 0 & 0 & 0 & 32 \\
\hline 16 & 17 & -1 & 6 & 0 & -58 & 0 & -3 & 29 & -31 \\
\hline 17 & -4 & 0 & -2 & 0 & -44 & 2 & -2 & 0 & -42 \\
\hline 18 & 1 & -12 & 12 & 0 & -38 & 0 & -6 & 19 & 23 \\
\hline 19 & -38 & 0 & 11 & 39 & -31 & -4 & 4 & -39 & 18 \\
\hline 20 & -24 & 4 & -12 & 0 & 132 & 0 & 6 & -66 & 80 \\
\hline 21 & 14 & 0 & -5 & 0 & 49 & 1 & -1 & 0 & 30 \\
\hline 22 & -16 & 4 & -12 & 0 & -12 & 0 & 6 & 6 & -56 \\
\hline 23 & 32 & 0 & 4 & 0 & 76 & 8 & -8 & 0 & 48 \\
\hline 24 & 18 & -2 & 4 & 0 & -12 & 0 & -2 & 6 & 18 \\
\hline 25 & 12 & 0 & -6 & -32 & -11 & 2 & -2 & 32 & -29 \\
\hline 26 & 24 & 2 & -6 & 0 & -70 & 0 & 3 & 35 & -60 \\
\hline 27 & 4 & 0 & 14 & -70 & -166 & 4 & -4 & 70 & -156 \\
\hline 28 & 21 & -7 & 10 & 0 & -98 & 0 & -5 & 49 & -39 \\
\hline
\end{tabular}

Theorem 4.1.1. For $(i, j)=(4,4),(6,2),(2,6)$, we have $\varphi^{i}(q) \varphi^{j}\left(q^{7}\right) \in M_{4}\left(\Gamma_{0}(28)\right)$. 
Proof. Appealing to (2.1.9) we then check conditions (L1), (L2) and (L3) of Theorem 3.1.1 for each quadratic form. We have $N=28$. First

$$
\varphi^{4}(q) \varphi^{4}\left(q^{7}\right)=\frac{\eta^{20}(2 z) \eta^{20}(14 z)}{\eta^{8}(z) \eta^{8}(4 z) \eta^{8}(7 z) \eta^{8}(28 z)} .
$$

Then we have

Table 4.4.2(a)

\begin{tabular}{r|rrrrrr}
\hline$\delta$ & 1 & 2 & 4 & 7 & 14 & 28 \\
\hline$r_{\delta}$ & -8 & 20 & -8 & -8 & 20 & -8 \\
\hline
\end{tabular}

It can be seen from Table 4.4.2(a) that conditions $(L 1)$ and $(L 2)$ are satisfied.

\begin{tabular}{r|rrrrrr} 
Table $4.4 .2(\mathrm{~b})$ \\
\hline$d \mid 28$ & 1 & 2 & 4 & 7 & 14 & 28 \\
\hline$\sum_{1 \leq \delta \mid 28} \frac{\operatorname{gcd}(d, \delta)^{2} \cdot r_{\delta}}{\delta}$ & 0 & $192 / 7$ & 0 & 0 & 192 & 0 \\
\hline
\end{tabular}

From Table 4.4.2(b) the condition (L3) is also satisfied. Thus $\varphi^{4}(q) \varphi^{4}\left(q^{7}\right) \in M_{4}\left(\Gamma_{0}(28)\right)$. Secondly we have

$$
\varphi^{2}(q) \varphi^{6}\left(q^{7}\right)=\frac{\eta^{10}(2 z) \eta^{30}(14 z)}{\eta^{4}(z) \eta^{4}(4 z) \eta^{12}(7 z) \eta^{12}(28 z)}
$$

Then we have

$$
\text { Table 4.4.3(a) }
$$

\begin{tabular}{r|rrrrrr}
\hline$\delta$ & 1 & 2 & 4 & 7 & 14 & 28 \\
\hline$r_{\delta}$ & -4 & 10 & -4 & -12 & 30 & -12 \\
\hline
\end{tabular}

It can be seen from Table 4.4.3(a) that conditions (L1) and (L2) are satisfied. 
Table 4.4.3(b)

\begin{tabular}{r|rrrrrr}
\hline$d \mid 28$ & 1 & 2 & 4 & 7 & 14 & 28 \\
\hline$\sum_{1 \leq \delta \mid 28} \frac{\operatorname{gcd}(d, \delta)^{2} \cdot r_{\delta}}{\delta}$ & 0 & $120 / 7$ & 0 & 0 & 264 & 0 \\
\hline
\end{tabular}

From Table 4.4.3(b) the condition (L3) is also satisfied. Thus $\varphi^{2}(q) \varphi^{6}\left(q^{7}\right) \in M_{4}\left(\Gamma_{0}(28)\right)$.

Thirdly we have

$$
\varphi^{6}(q) \varphi^{2}\left(q^{7}\right)=\frac{\eta^{30}(2 z) \eta^{10}(14 z)}{\eta^{12}(z) \eta^{12}(4 z) \eta^{4}(7 z) \eta^{4}(28 z)}
$$

Then we have

Table 4.4.4(a)

\begin{tabular}{r|rrrrrr}
\hline$\delta$ & 1 & 2 & 4 & 7 & 14 & 28 \\
\hline$r_{\delta}$ & -12 & 30 & -12 & -4 & 10 & -4 \\
\hline
\end{tabular}

It can be seen from Table 4.4.4(a) that conditions (L1) and (L2) are satisfied.

Table 4.4.4(b)

\begin{tabular}{r|rrrrrr}
\hline$d \mid 28$ & 1 & 2 & 4 & 7 & 14 & 28 \\
\hline$\sum_{1 \leq \delta \mid 28} \frac{\operatorname{gcd}(d, \delta)^{2} \cdot r_{\delta}}{\delta}$ & 0 & $264 / 7$ & 0 & 0 & 120 & 0 \\
\hline
\end{tabular}

From Table 4.4.4(b) the condition (L3) is also satisfied. Thus $\varphi^{6}(q) \varphi^{2}\left(q^{7}\right) \in$ $M_{4}\left(\Gamma_{0}(28)\right)$.

Theorem 4.1.2. $V_{k}(q)(1 \leq k \leq 9)$ given by $(4.1 .1)-(4.1 .9)$ are in $S_{4}\left(\Gamma_{0}(28)\right)$.

Proof. We will check conditions (L1), (L2) and (L4) of Theorem 3.1.1. We have $N=28$. Firstly we have

$$
V_{1}(q)=\eta^{2}(z) \eta^{2}(2 z) \eta^{2}(7 z) \eta^{2}(14 z)
$$

Then we have 
Table 4.4.5(a)

\begin{tabular}{r|rrrr}
\hline$\delta$ & 1 & 2 & 7 & 14 \\
\hline$r_{\delta}$ & 2 & 2 & 2 & 2 \\
\hline
\end{tabular}

It can be seen from Table 4.4.5(a) that conditions $(L 1)$ and $(L 2)$ are satisfied.

Table 4.4.5(b)

\begin{tabular}{r|rrrrrr}
\hline$d \mid 28$ & 1 & 2 & 4 & 7 & 14 & 28 \\
\hline$\sum_{1 \leq \delta \mid 28} \frac{\operatorname{gcd}(d, \delta)^{2} \cdot r_{\delta}}{\delta}$ & $24 / 7$ & $48 / 7$ & $48 / 7$ & 24 & 48 & 48 \\
\hline
\end{tabular}

From Table 4.4.5(b) the condition $(L 4)$ is also satisfied. Thus $V_{1}(q) \in S_{4}\left(\Gamma_{0}(28)\right)$.

Secondly we consider

$$
V_{2}(q)=\eta^{2}(2 z) \eta^{2}(4 z) \eta^{2}(14 z) \eta^{2}(28 z)
$$

Then we have

Table 4.4.6(a)

\begin{tabular}{r|rrrr}
\hline$\delta$ & 2 & 4 & 14 & 28 \\
\hline$r_{\delta}$ & 2 & 2 & 2 & 2 \\
\hline
\end{tabular}

It can be seen from Table 4.4.6(a) that conditions $(L 1)$ and $(L 2)$ are satisfied.

Table 4.4.6(b)

\begin{tabular}{r|rrrrrr}
\hline$d \mid 28$ & 1 & 2 & 4 & 7 & 14 & 28 \\
\hline$\sum_{1 \leq \delta \mid 28} \frac{\operatorname{gcd}(d, \delta)^{2} \cdot r_{\delta}}{\delta}$ & $12 / 7$ & $48 / 7$ & $96 / 7$ & 12 & 48 & 96 \\
\hline
\end{tabular}

From Table 4.4.6(b) the condition $(L 4)$ is also satisfied. Thus $V_{2}(q) \in S_{4}\left(\Gamma_{0}(28)\right)$.

Thirdly we consider

$$
V_{3}(q)=\eta^{2}(z) \eta^{2}(7 z) \eta^{4}(14 z)
$$

Then we have 
Table $4.4 .7(\mathrm{a})$

\begin{tabular}{r|rrr}
\hline$\delta$ & 1 & 7 & 14 \\
\hline$r_{\delta}$ & 2 & 2 & 4 \\
\hline
\end{tabular}

It can be seen from Table 4.4.7(a) that conditions $(L 1)$ and $(L 2)$ are satisfied.

\begin{tabular}{r|rrrrrr} 
Table $4.4 .7(\mathrm{~b})$ \\
\hline$d \mid 28$ & 1 & 2 & 4 & 7 & 14 & 28 \\
\hline$\sum_{1 \leq \delta \mid 28} \frac{\operatorname{gcd}(d, \delta)^{2} \cdot r_{\delta}}{\delta}$ & $18 / 7$ & $24 / 7$ & $24 / 7$ & 30 & 72 & 72 \\
\hline
\end{tabular}

From Table 4.4.7(b) the condition $(L 4)$ is also satisfied. Thus $V_{3}(q) \in S_{4}\left(\Gamma_{0}(28)\right)$.

Fourthly we consider

$$
V_{4}(q)=\eta^{4}(2 z) \eta^{2}(4 z) \eta^{2}(28 z)
$$

Then we have

Table 4.4.8(a)

\begin{tabular}{r|rrr}
\hline$\delta$ & 2 & 4 & 28 \\
\hline$r_{\delta}$ & 4 & 2 & 2 \\
\hline
\end{tabular}

It can be seen from Table 4.4.8(a) that conditions (L1) and (L2) are satisfied.

Table $4.4 .8(\mathrm{~b})$

\begin{tabular}{r|rrrrrr}
\hline$d \mid 28$ & 1 & 2 & 4 & 7 & 14 & 28 \\
\hline$\sum_{1 \leq \delta \mid 28} \frac{\operatorname{gcd}(d, \delta)^{2} \cdot r_{\delta}}{\delta}$ & $18 / 7$ & $72 / 7$ & $120 / 7$ & 6 & 24 & 72 \\
\hline
\end{tabular}

From Table $4.4 .8(\mathrm{~b})$ the condition $(L 4)$ is also satisfied. Thus $V_{4}(q) \in S_{4}\left(\Gamma_{0}(28)\right)$.

Fifthly we consider

$$
V_{5}(q)=\eta^{2}(z) \eta^{4}(2 z) \eta^{2}(7 z)
$$

Then we have 
Table 4.4.9(a)

\begin{tabular}{r|lll}
\hline$\delta$ & 1 & 2 & 7 \\
\hline$r_{\delta}$ & 2 & 4 & 2 \\
\hline
\end{tabular}

It can be seen from Table 4.4.9(a) that conditions $(L 1)$ and $(L 2)$ are satisfied.

Table $4.4 .9(\mathrm{~b})$

\begin{tabular}{r|rrrrrr}
\hline$d \mid 28$ & 1 & 2 & 4 & 7 & 14 & 28 \\
\hline$\sum_{1 \leq \delta \mid 28} \frac{\operatorname{gcd}(d, \delta)^{2} \cdot r_{\delta}}{\delta}$ & $30 / 7$ & $72 / 7$ & $72 / 7$ & 18 & 24 & 24 \\
\hline
\end{tabular}

From Table 4.4.9(b) the condition $(L 4)$ is also satisfied. Thus $V_{5}(q) \in S_{4}\left(\Gamma_{0}(28)\right)$. Sixthly we consider

$$
V_{6}(q)=\eta^{2}(4 z) \eta^{4}(14 z) \eta^{2}(28 z)
$$

Then we have

\begin{tabular}{r|rrr}
\multicolumn{4}{c}{ Table $4.4 .10(\mathrm{a})$} \\
\hline$\delta$ & 4 & 14 & 28 \\
\hline$r_{\delta}$ & 2 & 4 & 2 \\
\hline
\end{tabular}

It can be seen from Table 4.4.10(a) that conditions (L1) and (L2) are satisfied.

Table 4.4.10(b)

\begin{tabular}{r|rrrrrr}
\hline$d \mid 28$ & 1 & 2 & 4 & 7 & 14 & 28 \\
\hline$\sum_{1 \leq \delta \mid 28} \frac{\operatorname{gcd}(d, \delta)^{2} \cdot r_{\delta}}{\delta}$ & $6 / 7$ & $24 / 7$ & $72 / 7$ & 18 & 72 & 120 \\
\hline
\end{tabular}

From Table 4.4.10(b) the condition $(L 4)$ is also satisfied. Thus $V_{6}(q) \in S_{4}\left(\Gamma_{0}(28)\right)$.

Seventhly we have

$$
V_{7}(q)=\eta(z) \eta(4 z) \eta(7 z) \eta^{4}(14 z) \eta(28 z)
$$

Then we have 
Table 4.4.11(a)

\begin{tabular}{r|rrrrr}
\hline$\delta$ & 1 & 4 & 7 & 14 & 28 \\
\hline$r_{\delta}$ & 1 & 1 & 1 & 4 & 1 \\
\hline
\end{tabular}

It can be seen from Table 4.4.11(a) that conditions (L1) and (L2) are satisfied.

Table 4.4.11(b)

\begin{tabular}{r|rrrrrr}
\hline$d \mid 28$ & 1 & 2 & 4 & 7 & 14 & 28 \\
\hline$\sum_{1 \leq \delta \mid 28} \frac{\operatorname{gcd}(d, \delta)^{2} \cdot r_{\delta}}{\delta}$ & $12 / 7$ & $24 / 7$ & $48 / 7$ & 24 & 72 & 96 \\
\hline
\end{tabular}

From Table 4.4.11(b) the condition ( $L 4)$ is also satisfied. Thus $V_{7}(q) \in S_{4}\left(\Gamma_{0}(28)\right)$.

Eighthly we consider

$$
V_{8}(q)=\eta(z) \eta^{4}(2 z) \eta(4 z) \eta(7 z) \eta(28 z)
$$

Then we have

Table 4.4.12(a)

\begin{tabular}{r|rrrrr}
\hline$\delta$ & 1 & 2 & 4 & 7 & 28 \\
\hline$r_{\delta}$ & 1 & 4 & 1 & 1 & 1 \\
\hline
\end{tabular}

It can be seen from Table 4.4.12(a) that conditions (L1) and (L2) are satisfied.

Table 4.4.12(b)

\begin{tabular}{r|rrrrrr}
\hline$d \mid 28$ & 1 & 2 & 4 & 7 & 14 & 28 \\
\hline$\sum_{1 \leq \delta \mid 28} \frac{\operatorname{gcd}(d, \delta)^{2} \cdot r_{\delta}}{\delta}$ & $24 / 7$ & $72 / 7$ & $96 / 7$ & 12 & 24 & 24 \\
\hline
\end{tabular}

From Table 4.4.12(b) the condition $(L 4)$ is also satisfied. Thus $V_{8}(q) \in S_{4}\left(\Gamma_{0}(28)\right)$.

Ninthly we consider

$$
V_{9}(q)=\frac{\eta(z) \eta(2 z) \eta^{9}(7 z)}{\eta^{3}(14 z)}
$$

Then we have 
Table 4.4.13(a)

\begin{tabular}{r|rrrr}
\hline$\delta$ & 1 & 2 & 7 & 14 \\
\hline$r_{\delta}$ & 1 & 1 & 9 & -3 \\
\hline
\end{tabular}

It can be seen from Table 4.4.13(a) that conditions $(L 1)$ and $(L 2)$ are satisfied.

Table 4.4.13(b)

\begin{tabular}{r|rrrrrr}
\hline$d \mid 28$ & 1 & 2 & 4 & 7 & 14 & 28 \\
\hline$\sum_{1 \leq \delta \mid 28} \frac{\operatorname{gcd}(d, \delta)^{2} \cdot r_{\delta}}{\delta}$ & $18 / 7$ & $24 / 7$ & $24 / 7$ & 54 & 24 & 24 \\
\hline
\end{tabular}

From Table 4.4.13(b) the condition $(L 4)$ is also satisfied. Thus $V_{9}(q) \in$ $S_{4}\left(\Gamma_{0}(28)\right)$

Theorem 4.1.3. (a) $\left\{V_{1}(q), \ldots, V_{9}(q)\right\}$ constitute a basis for $S_{4}\left(\Gamma_{0}(28)\right)$.

(b) $E_{4}\left(q^{k}\right)(k=1,2,4,7,14,28)$ constitute a basis for $E_{4}\left(\Gamma_{0}(28)\right)$.

(c) $E_{4}\left(q^{k}\right)(k=1,2,4,7,14,28)$ together with $V_{k}(q)(1 \leq k \leq 9)$ constitute a basis for $M_{4}\left(\Gamma_{0}(28)\right)$.

Proof. (a) By Theorem 4.1.2, $V_{k}(q)(1 \leq k \leq 9) \in S_{4}\left(\Gamma_{0}(28)\right)$. There is no linear relationship among them. By Proposition 2.3.1, we have $\operatorname{dim} S_{4}\left(\Gamma_{0}(28)\right)=9$. Thus $V_{k}(q)(1 \leq k \leq 9)$ constitute a basis for $S_{4}\left(\Gamma_{0}(28)\right)$.

(b) By Proposition 2.3.1, we have $\operatorname{dim} E_{4}\left(\Gamma_{0}(28)\right)=6$. By Theorem 2.2.3, $E_{4}\left(q^{k}\right)$ $(k=1,2,4,7,14,28)$ constitute a basis for $E_{4}\left(\Gamma_{0}(28)\right)$.

(c) It follows from (a), (b) and (2.1.1) that the dimension of $M_{4}\left(\Gamma_{0}(28)\right)$ is 15 and therefore $E_{4}\left(q^{k}\right)(k=1,2,4,7,14,28)$ together with $V_{k}(q)(1 \leq k \leq 9)$ constitute a basis for $M_{4}\left(\Gamma_{0}(28)\right)$. 


\section{Theorem 4.1.4.}

(a) $\varphi^{4}(q) \varphi^{4}\left(q^{7}\right)=\frac{8}{25} E_{4}(q)-\frac{16}{25} E_{4}\left(q^{2}\right)+\frac{128}{25} E_{4}\left(q^{4}\right)+\frac{392}{25} E_{4}\left(q^{7}\right)-\frac{784}{25} E_{4}\left(q^{14}\right)$

$$
\begin{aligned}
& +\frac{6272}{25} E_{4}\left(q^{28}\right)-\frac{448}{25} V_{1}(q)+\frac{3712}{25} V_{2}(q)-\frac{544}{5} V_{3}(q)-\frac{768}{25} V_{4}(q) \\
& -\frac{1184}{25} V_{5}(q)+\frac{5376}{25} V_{6}(q)+\frac{1376}{25} V_{9}(q),
\end{aligned}
$$

(b) $\varphi^{2}(q) \varphi^{6}\left(q^{7}\right)=\frac{4}{75} E_{4}(q)-\frac{64}{75} E_{4}\left(q^{4}\right)-\frac{1204}{75} E_{4}\left(q^{7}\right)+\frac{19264}{75} E_{4}\left(q^{28}\right)-\frac{104}{25} V_{1}(q)$

$$
\begin{aligned}
& +\frac{416}{25} V_{2}(q)+\frac{728}{75} V_{3}(q)+\frac{1184}{75} V_{4}(q)+\frac{296}{75} V_{5}(q)+\frac{2912}{75} V_{6}(q) \\
& +\frac{2224}{75} V_{7}(q)+\frac{1168}{75} V_{8}(q),
\end{aligned}
$$

(c) $\varphi^{6}(q) \varphi^{2}\left(q^{7}\right)=\frac{172}{75} E_{4}(q)-\frac{2752}{75} E_{4}\left(q^{4}\right)-\frac{1372}{75} E_{4}\left(q^{7}\right)+\frac{21952}{75} E_{4}\left(q^{28}\right)+\frac{728}{25} V_{1}(q)$

$$
\begin{aligned}
& -\frac{2912}{25} V_{2}(q)+\frac{14504}{75} V_{3}(q)+\frac{2912}{75} V_{4}(q)+\frac{728}{75} V_{5}(q) \\
& +\frac{58016}{75} V_{6}(q)+\frac{57232}{75} V_{7}(q)+\frac{2224}{75} V_{8}(q) .
\end{aligned}
$$

Proof. By Theorem 4.1.1, we have $\varphi^{2 i}(q) \varphi^{2 j}\left(q^{7}\right) \in M_{4}\left(\Gamma_{0}(28)\right)$. Therefore by Theorem 4.1.3 $\varphi^{2 i}(q) \varphi^{2 j}\left(q^{7}\right)$ must be a linear combination of $E_{4}\left(q^{k}\right)(k=1,2,4$, $7,14,28)$ and $V_{k}(q)(1 \leq k \leq 9)$, namely

$$
\begin{aligned}
\varphi^{i}(q) \varphi^{j}\left(q^{7}\right)=x_{1} & E_{4}(q)+x_{2} E_{4}\left(q^{2}\right)+x_{3} E_{4}\left(q^{4}\right)+x_{4} E_{4}\left(q^{7}\right)+x_{5} E_{4}\left(q^{14}\right)+x_{6} E_{4}\left(q^{28}\right) \\
& +y_{1} V_{1}(q)+y_{2} V_{2}(q)+y_{3} V_{3}(q)+y_{4} V_{4}(q)+y_{5} V_{5}(q)+y_{6} V_{6}(q) \\
& +y_{7} V_{7}(q)+y_{8} V_{8}(q)+y_{9} V_{9}(q) .
\end{aligned}
$$

We equate the first twenty-eight coefficients of $q^{n}$ on both sides of the equation above to obtain a system of linear equations with the unknowns $x_{1}, x_{2}, x_{3}, x_{4}, x_{5}, x_{6}$ and $y_{1}, y_{2}, y_{3}, y_{4}, y_{5}, y_{6}, y_{7}, y_{8}, y_{9}$. Then, using MAPLE we solve the system to find the asserted coefficients. 
Theorem 4.1.5. Let $n \in \mathbb{N}$. Then

(a) $N\left(1^{4}, 7^{4} ; n\right)=\frac{8}{25} \sigma_{3}(n)-\frac{16}{25} \sigma_{3}(n / 2)+\frac{128}{25} \sigma_{3}(n / 4)+\frac{392}{25} \sigma_{3}(n / 7)$

$$
\begin{aligned}
& -\frac{784}{25} \sigma_{3}(n / 14)+\frac{6272}{25} \sigma_{3}(n / 28)-\frac{448}{25} v_{1}(n)+\frac{3712}{25} v_{2}(n) \\
& -\frac{544}{5} v_{3}(n)-\frac{768}{25} v_{4}(n)-\frac{1184}{25} v_{5}(n)+\frac{5376}{25} v_{6}(n)+\frac{1376}{25} v_{9}(n),
\end{aligned}
$$

(b) $N\left(1^{2}, 7^{6} ; n\right)=\frac{4}{75} \sigma_{3}(n)-\frac{64}{75} \sigma_{3}(n / 4)-\frac{1204}{75} \sigma_{3}(n / 7)+\frac{19264}{75} \sigma_{3}(n / 28)$

$$
\begin{aligned}
- & \frac{104}{25} v_{1}(n)+\frac{416}{25} v_{2}(n)+\frac{728}{75} v_{3}(n)+\frac{1184}{75} v_{4}(n)+\frac{296}{75} v_{5}(n) \\
& +\frac{2912}{75} v_{6}(n)+\frac{2224}{75} v_{7}(n)+\frac{1168}{75} v_{8}(n),
\end{aligned}
$$

(c) $N\left(1^{6}, 7^{2} ; n\right)=\frac{172}{75} \sigma_{3}(n)-\frac{2752}{75} \sigma_{3}(n / 4)-\frac{1372}{75} \sigma_{3}(n / 7)+\frac{21952}{75} \sigma_{3}(n / 28)$

$$
\begin{aligned}
& +\frac{728}{25} v_{1}(n)-\frac{2912}{25} v_{2}(n)+\frac{14504}{75} v_{3}(n)+\frac{2912}{75} v_{4}(n)+\frac{728}{75} v_{5}(n) \\
& +\frac{58016}{75} v_{6}(n)+\frac{57232}{75} v_{7}(n)+\frac{2224}{75} v_{8}(n) .
\end{aligned}
$$

Proof. Appealing (2.1.7), (2.2.4) and Theorem 4.1.4, we obtain

$$
\text { (a) } \begin{aligned}
\sum_{n=0}^{\infty} & N\left(1^{4}, 7^{4} ; n\right) q^{n}=\varphi^{4}(q) \varphi^{4}\left(q^{7}\right) \\
= & \frac{8}{25} E_{4}(q)-\frac{16}{25} E_{4}\left(q^{2}\right)+\frac{128}{25} E_{4}\left(q^{4}\right)+\frac{392}{25} E_{4}\left(q^{7}\right)-\frac{784}{25} E_{4}\left(q^{14}\right) \\
& +\frac{6272}{25} E_{4}\left(q^{28}\right)-\frac{448}{25} V_{1}(q)+\frac{3712}{25} V_{2}(q)-\frac{544}{5} V_{3}(q)-\frac{768}{25} V_{4}(q) \\
& -\frac{1184}{25} V_{5}(q)+\frac{5376}{25} V_{6}(q)+\frac{1376}{25} V_{9}(q), \\
= & 1+\sum_{n=1}^{\infty}\left(\frac{8}{25} \sigma_{3}(n)-\frac{16}{25} \sigma_{3}(n / 2)+\frac{128}{25} \sigma_{3}(n / 4)+\frac{392}{25} \sigma_{3}(n / 7)\right. \\
& -\frac{784}{25} \sigma_{3}(n / 14)+\frac{6272}{25} \sigma_{3}(n / 28)-\frac{448}{25} v_{1}(n)+\frac{3712}{25} v_{2}(n)-\frac{544}{5} v_{3}(n) \\
& \left.-\frac{768}{25} v_{4}(n)-\frac{1184}{25} v_{5}(n)+\frac{5376}{25} v_{6}(n)+\frac{1376}{25} v_{9}(n)\right) q^{n},
\end{aligned}
$$




$$
\text { (b) } \begin{aligned}
& \sum_{n=0}^{\infty} N\left(1^{2}, 7^{6} ; n\right) q^{n}=\varphi^{2}(q) \varphi^{6}\left(q^{7}\right) \\
&= \frac{4}{75} E_{4}(q)-\frac{64}{75} E_{4}\left(q^{4}\right)-\frac{1204}{75} E_{4}\left(q^{7}\right)+\frac{19264}{75} E_{4}\left(q^{28}\right)-\frac{104}{25} V_{1}(q) \\
&+\frac{416}{25} V_{2}(q)+\frac{728}{75} V_{3}(q)+\frac{1184}{75} V_{4}(q)+\frac{296}{75} V_{5}(q)+\frac{2912}{75} V_{6}(q) \\
&+\frac{2224}{75} V_{7}(q)+\frac{1168}{75} V_{8}(q), \\
&= 1+\sum_{n=1}^{\infty}\left(\frac{4}{75} \sigma_{3}(n)-\frac{64}{75} \sigma_{3}(n / 4)-\frac{1204}{75} \sigma_{3}(n / 7)+\frac{19264}{75} \sigma_{3}(n / 28)\right. \\
&-\frac{104}{25} v_{1}(n)+\frac{416}{25} v_{2}(n)+\frac{728}{75} v_{3}(n)+\frac{1184}{75} v_{4}(n)+\frac{296}{75} v_{5}(n) \\
&\left.+\frac{2912}{75} v_{6}(n)+\frac{2224}{75} v_{7}(n)+\frac{1168}{75} v_{8}(n)\right) q^{n}, \\
&(\mathbf{c}) \sum_{n=0}^{\infty} N\left(1^{6}, 7^{2} ; n\right) q^{n}=\varphi^{6}(q) \varphi^{2}\left(q^{7}\right) \\
&= \frac{172}{75} E_{4}(q)-\frac{2752}{75} E_{4}\left(q^{4}\right)-\frac{1372}{75} E_{4}\left(q^{7}\right)+\frac{21952}{75} E_{4}\left(q^{28}\right)+\frac{728}{25} V_{1}(q) \\
&+\frac{2912}{25} V_{2}(q)+\frac{14504}{75} V_{3}(q)+\frac{2912}{75} V_{4}(q)+\frac{728}{75} V_{5}(q)+\frac{58016}{75} V_{6}(q) \\
&+\frac{57232}{75} V_{7}(q)+\frac{2224}{75} V_{8}(q), \\
&= 1+\sum_{n=1}^{\infty}\left(\frac{172}{75} \sigma_{3}(n)-\frac{2752}{75} \sigma_{3}(n / 4)-\frac{1372}{75} \sigma_{3}(n / 7)+\frac{21952}{75} \sigma_{3}(n / 28)\right. \\
&+\frac{2912}{25} v_{2}(n)+\frac{14504}{75} v_{3}(n)+\frac{2912}{75} v_{4}(n)+\frac{728}{75} v_{5}(n) \\
&\left.+\frac{57232}{75} v_{7}(n)+\frac{2224}{75} v_{8}(n)\right) q^{n} . \\
& \\
&
\end{aligned}
$$

Equating the coefficients of $q^{n}$ on both sides of equations (a)-(c) yields the results.

\subsection{Conclusion and Future Work}

In this thesis we have determined formulae for the number of representations of positive integers by quadratic forms by using a modular form approach. We worked on 
quaternary and octonary quadratic forms with certain coefficients.

We first found the number of representations of positive integers by quaternary quadratic form $a_{1} x_{1}^{2}+a_{2} x_{2}^{2}+a_{3} x_{3}^{2}+a_{4} x_{4}^{2}$, where $a_{1}, a_{2}, a_{3}, a_{4} \in\{1,2,7,14\}$. We then extended our work to octonary quadratic forms, and determined explicit formulae for $N\left(1^{i}, 7^{j} ; n\right)$, where $(i, j)=(4,4),(6,2)$ and $(2,6)$. We plan to extend our work to the remainning octonary quadratic forms with coefficients 1, 2, 7 and 14 .

It would be natural to extend our work to find the number of representations of positive integers by quadratic forms with an odd number of variables. Our first step would be to start with ternary, quinary or septenary quadratic forms with coefficients 1, 2 and 7. For some recent work on this subject, one can see [5], [6] and [7].

Finally it would be interesting to find all the eta quotients in spaces $M_{2}\left(\Gamma_{0}(56), \chi\right)$ and $M_{4}\left(\Gamma_{0}(28), \chi\right)$, and study the properties of their Fourier coefficients. 


\section{Bibliography}

[1] A. Alaca, S. Alaca, M. F. Lemire and K. S. Williams, Nineteen quaternary quadratic forms, Acta Arith. 130 (2007), 277-310.

[2] A. Alaca, S.. Alaca and K. S. Williams, Fourteen octonary quadratic forms, Int. J. Number Theory 6 (2010), 37-50.

[3] B. C. Berndt, Number Theory in the Spirit of Ramanujan, Amer. Math. Soc, Providence, 2006.

[4] E. Benz, Über die Anzahl Darstellungen einer Zahln durch gewisse quaternäre quadratische Formen: Beweise, welche auf Identitäten aus dem Gebiete der Theta- funktionen basieren. Dissertation, Zürich 1964, in: Studien zur Theorie der quadratischen Formen (edited by B. L. van der Waerden und H. Gross), Stuttgart, 1968, pp. 165-198.

[5] S. Cooper and H. Y. Lam, On the Diophantine equation $n^{2}=x^{2}+b y^{2}+c z^{2}$, J. Number Theory 133 (2013), 719-737.

[6] S. Cooper, H. Y. Lam and D. Ye, Representations of squares by certain quinary quadratic forms, Acta Arith. 157 (2013), 147-168.

[7] S. Cooper, H. Y. Lam and D. Ye, Representations of squares by certain septenary quadratic forms, Integers. 13 (2013), A35, 18 pp. 
[8] F. Diamond and J. Shurman, A First Course in Modular Forms, Graduate Text in Mathematics 228, Springer-Verlag, 2004.

[9] F. Diamond and J. Im, Modular form and Modular curves, CMS Conf. Proc. 17 (1955).

[10] P. Demuth, Die Zahl der Darstellungen einer natürlichen Zahl durch spezielle quaternäre quadratische Formen aufgrund der Siegelschen Massformel, in: Studien zur Theorie der quadratischen Formen (edited by B. L. van der Waerden und H. Gross), Stuttgart, 1968, pp. 224-254.

[11] B. Gordon and D. Sinor, Multiplicative properties of $\eta$-products, Lecture Notes in Math, vol.1395 Springer-Verlag, New York (1989), 173-200.

[12] C. G. J. Jacobi, Fundamenta nova theoriae functionum ellipticarum, in Gesammelte Werke, Erster Band (Chelsea Publishing Co., New York, 1969), pp. 49-239.

[13] G. Köhler, Eta Products and Theta Series Identities, Springer Monographs in Mathematics, Springer, 2011.

[14] L. J. P. Kilford, Modular Forms, A classical and computational introduction, Imperial College Press, London, 2008.

[15] N. Koblitz, Introduction to Elliptic curves and Modular forms, Springer-Verlag, New York (1984).

[16] T. Koshy, Elementary Number Theory with Applications, Smarandache Notions Journal 13 (2002).

[17] G. Ligozat, Courbes modulaires de genre 1, Bull. Soc. Math. France 43 (1975), $5-80$. 
[18] J. Liouville, Sur les deux formes $x^{2}+y^{2}+2\left(z^{2}+t^{2}\right)$, J. Pures Appl. Math. 5 (1860), 269-272.

[19] M. Lemire, Extension of Ramanujan-Mordell formula, Ph.D. thesis, Carleton University (2010).

[20] L. J. Mordell, On the representations of numbers as a sum of 2r squares, Quart. J. Pure Appl. Math. 48 (1917), 93-104.

[21] T. Miyake, Modular Forms, Springer Monographs in Mathematics. SpringerVerlag, Berlin, english edition, 2006. Translated from the 1976 Japanese original by Yoshitaka Maeda.

[22] K. Ono, The Web of Modularity. Arithmetic of the coefficient of Modular Forms and q-Series, Am. Math. Soc, Providence, 2004.

[23] T. Pepin, Etude sur quelques formules d'analyse utiles dans la theorie des nombres, Atti Accad. Pont. Nuovi Lincei 38 (1884-5), 139-196.

[24] S. Ramanujan, On certain arithmetical functions, Trans. Cambridge Philos. Soc. 22 (1916), 159-184.

[25] L. Robledo, Elliptic Curves, Modular Forms, and their L-functions, Amer. Math. Soc, 2011 .

[26] B. K. Spearman and K. S. Williams, The simplest arithmetic proof of Jacobi's four squares theorem, Far East J . Math. Sci. (FJMS) 2 (2000), 433-439.

[27] J. P. Serre, A course in arithmetic, Springer-Verlag, New York, 1973.

[28] W. A. Stein, Modular Forms, A Computational Approach, Amer. Math. Soc, Graduate Studies in Mathematics 79 (2007). 
[29] K. S. Williams, On the representations of a positive integer by the forms $x^{2}+$ $y^{2}+z^{2}+2 t^{2}$ and $x^{2}+2 y^{2}+2 z^{2}+2 t^{2}$, Int. J. Modern Math. 3 (2008), 225-230.

[30] K. S. Wlliams, The simplest arithmetic proof of Jacobi's eight squares theorem, Far East J. Math. Sci. (FJMS) 3(6) (2001), 1001-1005. 